\title{
Sensing and stimulation of the vagus nerve for artificial cardiac control
}

Simone Ordelman 
Samenstelling promotiecommissie:

Voorzitter:

Secretaris:

Promotor:

Assistent promotor:

Leden:
Prof.dr.ir. A.J. Mouthaan

Prof.dr.ir. A.J. Mouthaan

Prof. dr. ir. P.H. Veltink

Dr. L. Kornet

Prof.dr.ir. E. Marani

Prof.dr.ir. C.H. Slump

Prof.dr. J.L.R.M. Smeets

Dr.ir. J.J. Struijk

Dr. H.P.J. Buschman
Universiteit Twente

Universiteit Twente

Universiteit Twente

Medtronic

Universiteit Twente

Universiteit Twente

Radboud Universiteit

Aalborg University,DK

Medtronic

Dit werk is gesponsord door Medtronic.

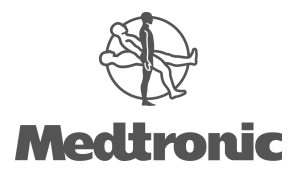

Gedrukt door Ipskamp Drukkers BV, Nijmegen, Nederland 


\title{
PROEFSCHRIFT
}

\author{
ter verkrijging van \\ de graad van doctor aan de Universiteit Twente, \\ op gezag van de rector magnificus, \\ prof. Dr. H. Brinksma, \\ volgens besluit van het College voor Promoties \\ in het openbaar te verdedigen \\ op vrijdag 27 januari 2012 om 16.45 uur
}

door

Simone Cornelia Maria Anna Ordelman geboren op 25 februari 1984 te Nijmegen 
Dit proefschrift is goedgekeurd door de promotor en assistent-promotor:

Prof. dr. ir. Peter H. Veltink

Dr. Lilian Kornet

ISBN: 978-90-365-3309-6

Copyright (C) 2011/2012, Simone Ordelman, 's-Hertogenbosch, the Netherlands 


\section{Content}

Chapter 1 General introduction 7

Chapter 2 Neural signals modulated with the cardiac cycle can 25

be measured extraneurally from the cervical vagus nerve

Chapter 3 An indirect component in the evoked compound action potential of the vagus nerve

Chapter 4 The average reference method for recording a dispersed evoked signal from peripheral nerves - a modelling study

Chapter 5 Additional experimental data on the origin of the late component in the evoked vagus nerve compound action potential

Chapter 6 Evoked responses from cranial origin in the cervical vagus 91 nerve

Chapter 7 The cardiac effects of vagus nerve stimulation

Chapter 8 Selectivity for specific cardiovascular effects of vagus nerve stimulation with a multi-contact electrode cuff

Chapter 9 Vagus nerve recording and stimulation in awake pigs

Chapter 10 General Discussion

Summary

Samenvatting

Dankwoord

Curriculum vitae 



\section{Chapter 1}

\section{General introduction}




\subsection{About this thesis}

To create a device that can seamlessly take over a malfunctioning part of the human body is one of the biggest challenges in biomedical engineering. In the past century, devices have been developed for bladder control, hearing, seeing, and insulin delivery, to name a few. The functional quality that such devices can deliver is steadily improving.

The system that is the focus of this thesis is the neurocardiac system. In patients with cardiovascular disease, this neurocardiac system can be impaired. The vagus nerve plays a vital role in neurocardiac control, and electrical stimulation of this nerve has been shown beneficial in certain cases [1-11]. This thesis will address not only this electrical stimulation, but also the relevant signals that can be recorded from the vagus nerve, which may be used as an input to a vagus nerve stimulator. With this input, stimulation parameters can be adjusted as needed to support the affected neurocardiac system in a closed-loop manner (figure 1.1).

In this first chapter, the relevant subjects are introduced. In the first paragraph, an overview is given of the pathways and mechanisms involved in neural control of the heart. In the following paragraph, possibilities and limitations of recording cardiac vagal signals are introduced. Next, the current applications of vagus nerve stimulation for cardiovascular effects are presented. The final paragraph of this chapter further outlines the thesis content.

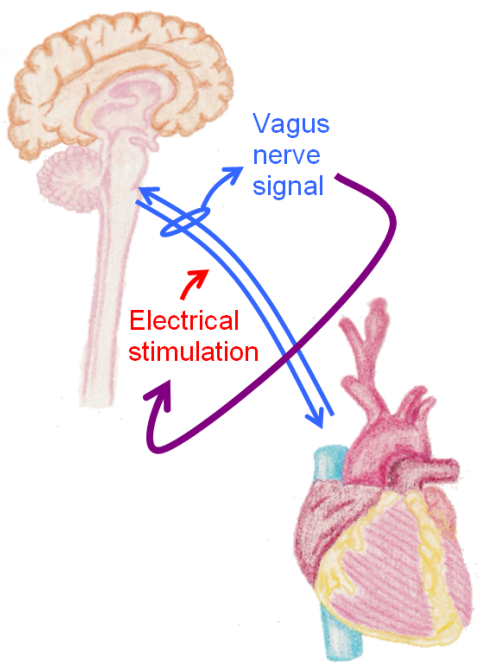

Figure 1.1: Schematic representation of artificial closed-loop neurocardiac control. Signals between the brain and the heart that are communicated via the vagus nerve are represented by the blue arrows. The purple arrow represents the closed loop: The signal recorded from the vagus nerve is used as an input to control electrical vagus nerve stimulation. 


\subsection{Neural control of the heart}

In a healthy individual, the heart can beat independently of any nervous influences. It generates an electrical signal at the sinoatrial (SA) node, giving rise to an atrial contraction, which is transmitted via the atrio-ventricular (AV) node to generate a ventricular contraction. The rate of this spontaneous rhythm of the heart is called the intrinsic heart rate. The autonomic (or vegetative) nervous system consisting of a parasympathetic and sympathetic component, regulates the cardiovascular parameters; heart rate (via the SA node), blood pressure, contractility of the heart, and AV-node conduction velocity (figure $1.2)$.

The sympathetic and parasympathetic limbs are generally considered antagonists [12-15]. The sympathetic system has an excitatory modulatory effect on the cardiovascular system and the parasympathetic an inhibitory modulatory effect. At rest, parasympathetic activation dominates and heart rate is somewhat below the intrinsic heart rate.

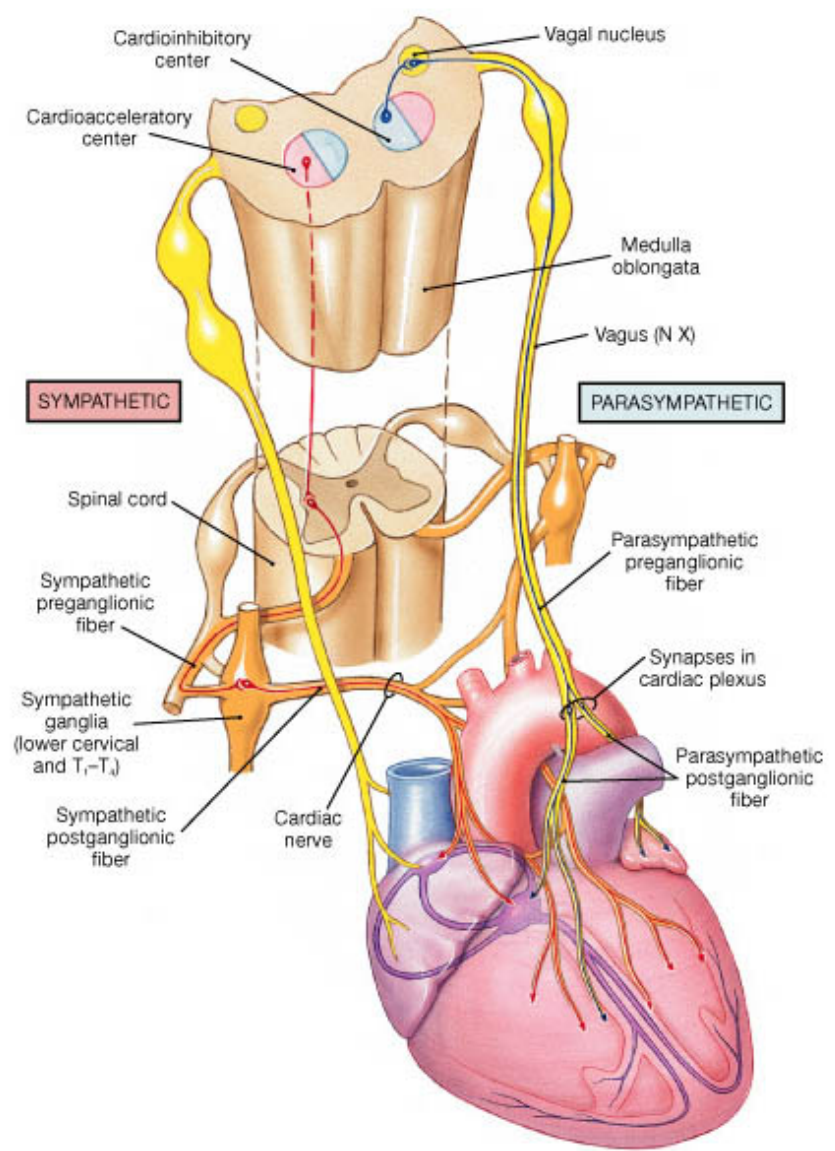

Figure 1.2: Autonomic innervation of the heart [16]. The main components of the sympathetic and parasympathetic system are indicated. 
Parasympathetic innervation of the heart is provided by the vagus nerve, the $\mathrm{X}^{\text {th }}$ cranial nerve (figure 1.2). Vagal preganglionic neurons arise in the medulla region of the brain stem and travel along the left and right vagus nerve. They synapse with neurons in terminal ganglia that are located within the target organs, called their intramural position [17-18]. The parasympathetic ganglionated plexus innervating the heart postganglionicly lie mainly embedded in epicardial fat pads [19]. These fat pads are located on the surface of the right and left atria, the left and right ventricle, the posterior wall near the ostia of the pulmonary veins, the vena cava, and the coronary sinus [19-22].

The sympathetic system has paravertebral ganglia close to the spinal cord and prevertebral intrathoracic and intra-abdominal ganglia. Preganglionic sympathetic neurons involved in neurocardiac control arise at thoracic levels from the spinal cord [17] and mostly synapse with long neurons in these ganglia. However, preganglionic sympathetic nerves also synapse in ganglia on the heart [23]. Preganglionic fibres, both of sympathetic and parasympathetic origin, and the parasympathetic postganglionic axons involved in neural control of the heart release the neurotransmitter acetylcholine, and are called cholinergic [17]. The sympathetic postganglionic axons release noradrenalin or adrenaline and are called adrenergic [17]. 


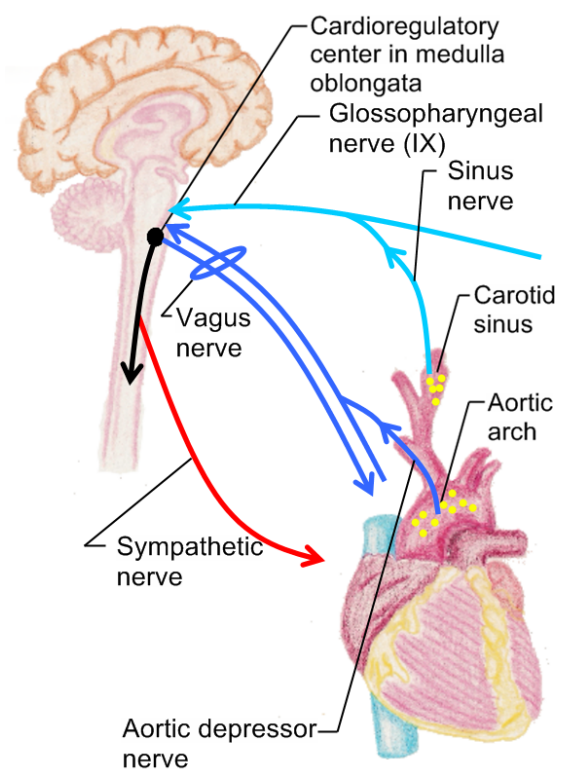

A: Baroreceptor reflex

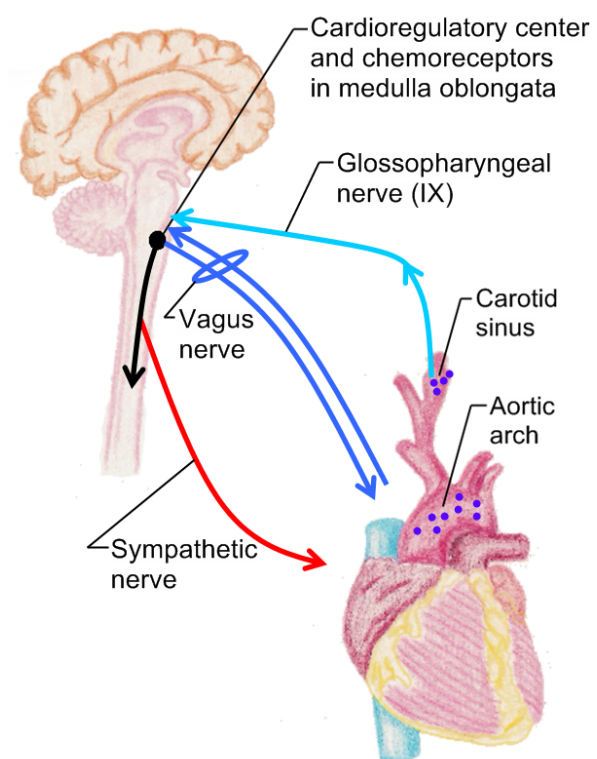

\section{C: Chemoreceptor reflex}

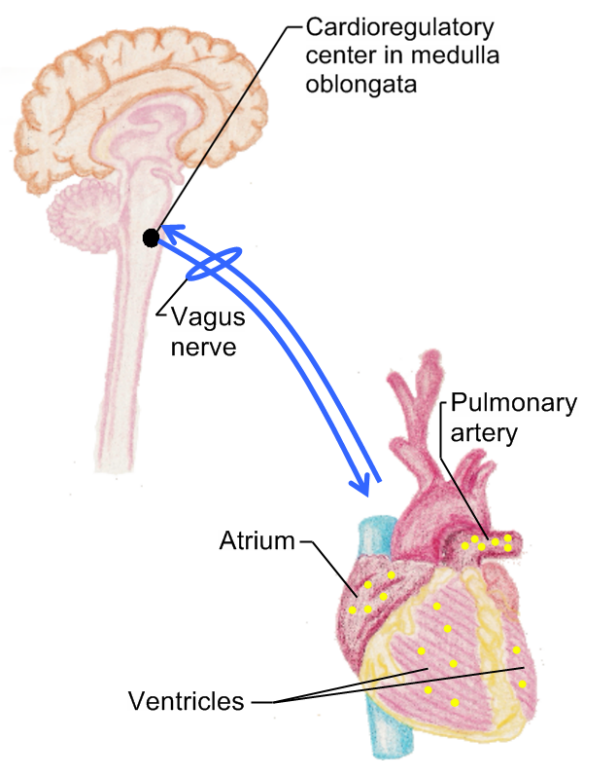

B: Bainbridge reflex

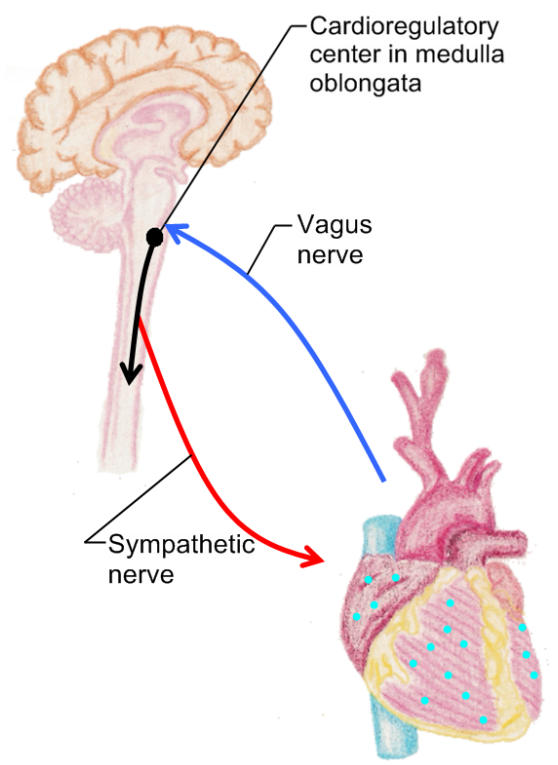

D: Bezold-Jarisch reflex

Figure 1.3: Schematic representation of reflexive control with feedback signals from cardiovascular receptors in the baroreceptor reflex (A), the Bainbridge reflex (B), the Chemoreceptor reflex $(C)$ and the Bezold-Jarisch reflex (D). 
Both the sympathetic and the parasympathetic system are reflexive in nature. These reflexes provide feedback control for the neurocardiac control system, with feedback signals arising from mechanoreceptors and chemoreceptors (figure 1.3).

Stress-sensitive baroreceptors (mechanoreceptors) are present in both the high and low pressure parts of the circulation. In the high-pressure part, where the blood goes from the heart into the circulation, the main baroreceptors are located in the carotid sinus and aortic arch [24-26]. Signals from the carotid sinus receptors are carried by the sinus nerve, which joins the glossopharyngeal nerve (IX ${ }^{\text {th }}$ cranial nerve) [17]. The axons from the aortic arch receptors are included in the aortic depressor nerve, an afferent branch of the vagus nerve [17]. The carotid sinus and aortic arch baroreceptors relay their signals via a mix of myelinated and unmyelinated axons. The receptors provide feedback for the primary regulation of the aortic pressure in the baroreceptor reflex. When blood pressure is low, the action potential rate of the receptors is low and heart rate is increased through the baroreceptor reflex.

In the low pressure part, in which blood is returned to the heart, mechanoreceptors are located in the junction of the atria with their veins, the pulmonary artery, the endocardium and the epicardium of the atria and ventricles [17]. Their signals travel via unmyelinated fibres of the vagus nerve and provide feedback for the Bainbridge reflex. The Bainbridge reflex [27] responds to increases of blood volume at the venous side of the circulation by increasing heart rate and ventricular contractility via inhibition of efferent vagal fibres. Increased heart rate and ventricular contractility decrease the blood volume at the venous side of the circulation by pumping more blood out of the right atrium. The Bainbridge reflex is a counterbalance to the baroreceptor reflex. The Bainbridge reflex is dominant when blood volume is increased, whereas the baroreceptor reflex is dominant when blood volume is decreased.

The chemoreceptor reflex is also involved in blood pressure control. Chemoreceptors are located peripherally near the baroreceptors in the carotid sinus and aortic arch, and located centrally in the medulla oblongata [17]. Afferent signals from carotid sinus chemoreceptors travel via the glossopharyngeal nerve and signals from the aortic arch chemoreceptors travel via the vagus nerve. The chemoreceptors have unmyelinated axons. The peripheral chemoreceptors predominantly sense arterial oxygen and carbon dioxide concentration, whereas the central receptors sense $\mathrm{pH}$ and carbon dioxide concentration $[15,28]$. In case of decreased blood oxygen, increased carbon dioxide or decreased $\mathrm{pH}$, the firing rate of the chemoreceptors is decreased. As a result, efferent vagus activity decreases and sympathetic activity increases, which increases heart rate, stroke volume and vasoconstriction.

Mixed sensors, which are sensitive to both mechanical and chemical stimuli, are present in the walls of all cardiac chambers [29]. Their information is transmitted by unmyelinated fibres in the vagus nerve. When activity from these 
mixed sensors is decreased, sympathetic output is increased through the BezoldJarisch reflex and blood pressure and heart rate are increased [29]. The effects of the Bezold-Jarisch reflex are attenuated by the baroreceptor reflex during normal conditions, i.e. the two reflexes are complementary in blood pressure control. The Bezold-Jarisch reflex can become dominant, as is the case when the receptors are activated by veratrum alkaloids [29].

In addition to feedback control via the brain stem, an extensive neural network on the heart is involved in neurocardiac control. This network consists of the interconnected ganglionated plexus on the epicardium of the heart. This complex neural network on the heart, also called 'the little brain on the heart' integrates afferent and efferent as well as parasympathetic and sympathetic activity as a final relay station, and is thought to be important in the coordinated neural control of the heart $[23,30]$. The heart is not the only organ with an intrinsic nervous system. Another example of an intrinsic nervous system is the enteric nervous system, which controls the activities of the digestive tract [3132]. Recently, an airway intrinsic nervous system has also been identified that controls airway muscle tone, gland secretion and blood flow [33].

\subsection{Recording cardiac vagal signals}

Cardiac signals from the vagus nerve may provide relevant information about the state of the cardiovascular system. Recording from the vagus nerve can be done at various levels. The neck region is clinically most easily accessible. This is therefore the region at which the vagus nerve is accessed for vagus nerve therapy in epilepsy. In the neck region, the vagus nerve is a thick trunk which contains fibres innervating many different organs. Only the branch to the pharynx and the superior branch to the larynx have branched off at this point (figure 1.4). The cervical vagus trunk at this point still contains the recurrent branch to the larynx and fibres going to liver, gallbladder, cardiovascular structures, stomach, small intestine, kidneys, pancreas and the cranial part of the large intestine. This means that when recording a signal in the neck region, the signal will contain information from many organs. Following the vagus nerve as it goes towards the heart, multiple branches that innervate the heart leave the nerve trunk of the left and right vagus nerve at different levels. These are the rami cardiaci craniales, medii and caudales. Additional cardiac branches arise from the trunk of the right vagus nerve at the level of the trachea, and from both recurrent laryngeal nerves after they leave the vagus nerves [18]. 


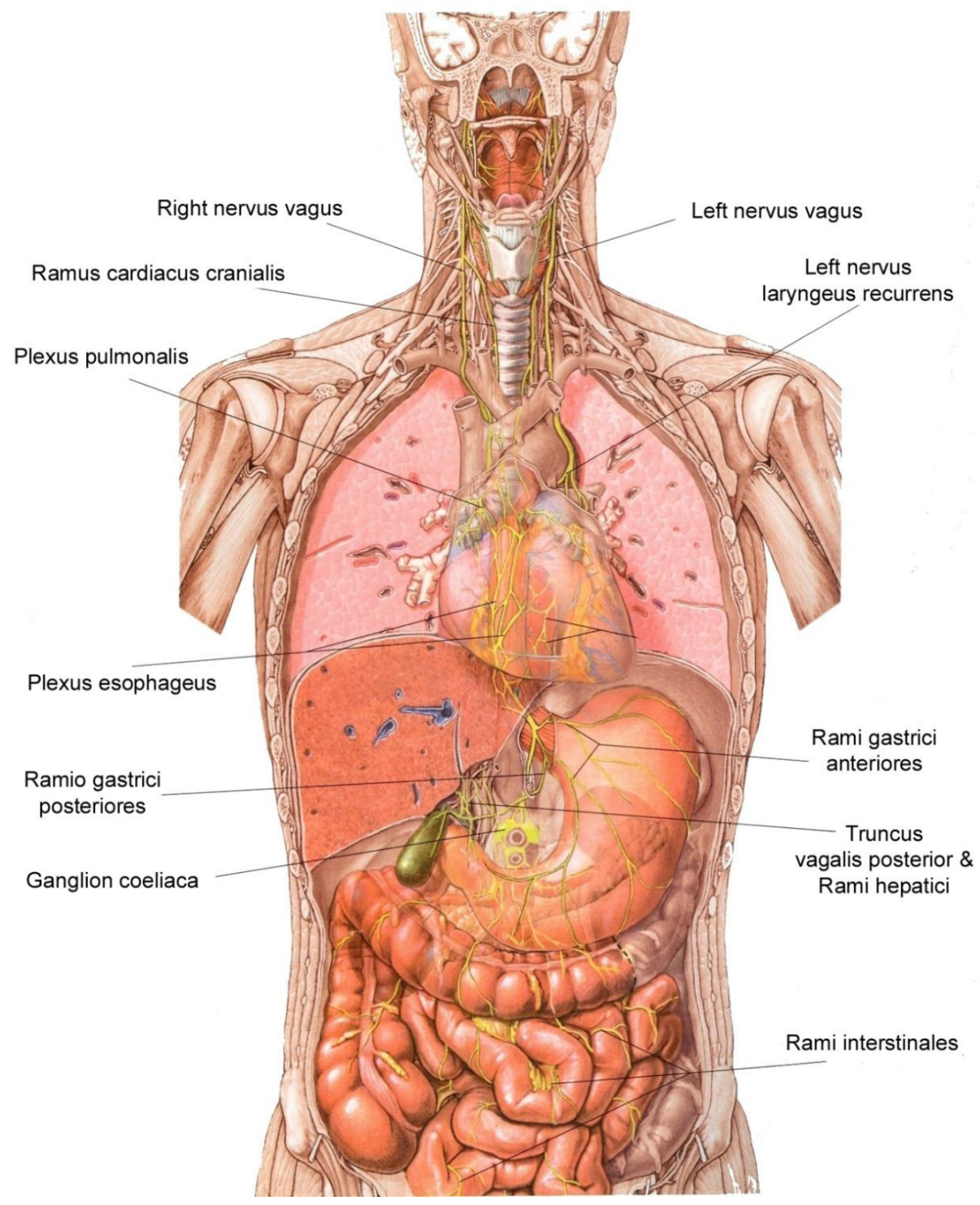

Figure 1.4: Representation of the vagus nerve branching [34]. 
Single fibre recordings have identified signals of cardiac efferent and afferent vagus nerve fibres [35-47]. When recording from the whole vagus nerve, selective detection of cardiac fibre signals is difficult. The vagus nerve contains the motor innervation of most of the smooth muscles and glands of the visceral organs and the striated muscles of the pharynx and larynx [18]. Cardiac signals will be obscured by signals coming from other fibre types when making whole nerve recordings. The thick, myelinated fibres generate an electrical signal with a higher amplitude and have a lower threshold for electrical stimulation than thin, unmyelinated fibres [48-49]. The fibre distribution of the cervical vagus nerve has been studied in the cat, rat and rabbit [42, 50-54]. About ten percent of all the fibres in the cat's cervical vagus nerve are cardiac fibres, and about 80 percent of those are afferent. A little over half of the cardiac afferent fibres are myelinated. The 20 percent efferent cardiac fibres are thin myelinated or unmyelinated fibres. There are more afferent than efferent myelinated cardiac fibres. Therefore, afferent cardiac fibres could more likely be recorded from the whole vagus nerve than efferent cardiac fibres. However, organization of the fibres within the nerve is also relevant. Signals from fibres located closest to the perimeter of the nerve, and thus closer to extraneural electrodes, will be recorded more easily than signals from fibres located in the centre of the nerve. The fibre organization within the vagus nerve may vary between subjects, and along the course of the nerve.

Recording cardiac signals from the whole cervical vagus nerve at the cervical level has been attempted in animal studies [55-57]. The neural activity recorded with intraneural bipolar electrodes showed some increased burst activity when blood pressure was increased in goats [57]. The neural activity of the rat, recorded with a bipolar hook electrode, showed a modulation with the ECG (Electro Cardio Gram, i.e. recorded electrical cardiac activity). In each cardiac cycle a similar pattern of increasing and decreasing vagus nerve activity was found [55]. The vagal activity showed an increase during the QRS complex of the ECG and a decrease directly afterwards. Instead of inspecting the direct recordings from the vagus nerve, the spectrum of the ENG (Electro Neuro Gram, i.e. recorded electrical neural activity) was used in a study using a spiral cuff around the nerve [56]. A peak in the spectrum of ENG recorded from the vagus nerve appeared when baroreceptors were influenced. The authors concluded that they obtained selective sensory information [56].

Recordings of the vagus nerve have also been made at a more caudal level, in the thorax above the aortic arch. In these recordings neural activity related to the cardiovascular system was found [58]. This activity was found to increase with heart failure and paroxysmal atrial tachycardia. These recordings were made with bipolar intraneural wires. At the thoracic level, part of the myelinated fibres have branched off and will not obscure the cardiac signals. However, a more invasive surgery is needed. 
Recordings of the vagus nerve may be used to detect diseased states, such as ischemia or arrhythmias. In patients with heart failure, the autonomic balance between the sympathetic and the parasympathetic system is disturbed. Parasympathetic withdrawal may contribute to the development of heart failure [59]. Heart failure affects the intrinsic cardiac nervous system's capacity to regulate the cardiovascular system [60]. An abnormality at the level of the ganglion on the surface of the heart is responsible for diminished parasympathetic control of the heart in heart failure [61].

Diminished cardiac vagal activity is apparent at an early stage of heart failure [62-64]. Using the rapid pacing canine model of heart failure, in which the heart is paced at a high rate, altered vagal control of heart rate was apparent within four days on initiation of pacing, at a time when cardiac function is impaired minimally [62]. Early action may reverse vagal dysfunction [65]. This means that if vagal dysfunction can be observed from vagus nerve recordings, action may be taken to impede heart failure development.

\subsection{Vagus nerve stimulation}

Electrical stimulation of the vagus nerve is an approved therapy for refractory epilepsy and depression $[1,5]$. Recently, vagus nerve stimulation (VNS) has emerged as a promising new therapy for various cardiac conditions $[2,7,9,11]$.

VNS reduces heart rate, ventricular contractility and blood pressure, and also has profound effects on cardiac electrophysiology and arrhythmogenesis. VNS therefore has the potential to be effective as a therapy for cardiac arrhythmias. In patients with tachycardia (increased heart rate) VNS may be used to reduce heart rate [66]. Increasing vagal tone by electrical stimulation may provide protective effects against ventricular arrhythmias [9, 67-68]. Some studies suggest that incidence of atrial fibrillation is reduced by VNS, but further studies are required on the subject [10].

VNS is currently being explored as a therapy for heart failure [3-4, 7-8, 11]. First studies in man have shown that VNS for heart failure is feasible, safe and tolerable [8]. Chronic VNS is potentially beneficial for quality of life and left ventricular volumes $[3,8]$. The beneficial effects of VNS may be attributed to several different mechanisms [59]: A reduced heart rate, increased heart rate variability, improved baroreflex sensitivity, direct antiarrhythmic effects, or antiinflammatory effects [69-70].

\subsection{Objectives of this thesis}

To obtain a closed-loop vagus nerve stimulator for neurocardiac control, the two main requirements are recording relevant cardiac signals from the vagus nerve and stimulating cardiac vagal fibres. 
The goal of this thesis can therefore be divided in two main objectives:

1. To sense relevant cardiac signals from the vagus nerve

2. To stimulate cardiac vagal fibres

Spontaneous neural activity of cardiac vagal fibres contains activity patterns that are related to the cardiac cycle. We hypothesized that these patterns can be recorded from whole nerve recordings at the cervical level of the vagus nerve. We expected to find patterns that change when cardiovascular parameters vary, because of related variations in cardiac receptor firing. Two electrode configurations were compared, one with three rings and one with twelve segments, in an attempt to improve selectivity for cardiac vagal fibres. This is described in chapter two.

When the vagus nerve is stimulated with electrical pulses, compound action potentials result from the fibres of the nerve. We hypothesized that evoked compound action potentials may contain components that are related to the cardiovascular system, coming via reflexive pathways of the neurocardiac control. In chapter three, these evoked signals are analyzed.

The method of recording indirect components of the evoked compound action potential is further analyzed, using a computer model. This model simulates action potentials that result from electrical stimulation of the vagus nerve, and calculates the signal that can be recorded at the recording electrode sites. The recording of an indirect component, which is a fast and dispersed signal, is modelled for various electrodes configurations. This model is described in chapter four.

The hypothesized relationship between the evoked compound action potential and the cardiovascular system is further examined by additional experiments in which the role of the recurrent laryngeal nerve was analyzed. This analysis is presented in chapter five.

A limitation of extraneural whole nerve recordings is that fibres with a small diameter at the centre of the nerve are difficult to record. These signals may be recorded using wire electrodes inserted into the nerve. Compound action potentials recorded with wire electrodes are compared to those recorded with extraneural cuffs in chapter six.

When the vagus nerve is electrically stimulated at a high enough frequency and amplitude, the state of the cardiovascular system is affected. Because of the reflexive nature of neurocardiac control, and sympathetic and parasympathetic interactions, the cardiovascular effects can be varied and complex. These effects are presented in chapter seven. 
Stimulating the vagus nerve at the cervical level can lead to various effects, depending on the vagal fibres that are excited. Selectivity of stimulation should therefore be attained. We hypothesized, that by applying the stimulation current to only a segment of the nerve, cardiovascular parameters can be influenced selectively. An electrode configuration is tested for this purpose in chapter eight.

Most of the experiments were performed in the anesthetized condition. Anaesthetics may influence the neurocardiac system and suppress neural signals. We hypothesized that in awake animals additional cardiac-related vagal signals may be found compared to the anesthetized condition. Experiments that were performed in freely moving awake animals are described in chapter nine.

This thesis is concluded with a general discussion in chapter ten.

\section{References}

1. Ben-Menachem, E. (2002) Vagus-nerve stimulation for the treatment of epilepsy Lancet Neurol, 1(8), p. 477-82.

2. Bilgutay, A.M., I.M. Bilgutay, F.K. Merkel, and C.W. Lillehei (1968) Vagal tuning. A new concept in the treatment of supraventricular arrhythmias, angina pectoris, and heart failure $\mathrm{J}$ Thorac Cardiovasc Surg, 56(1), p. 71-82.

3. De Ferrari, G.M., H.J. Crijns, M. Borggrefe, G. Milasinovic, J. Smid, M. Zabel, A. Gavazzi, A. Sanzo, R. Dennert, J. Kuschyk, S. Raspopovic, H. Klein, K. Swedberg, and P.J. Schwartz (2010) Chronic vagus nerve stimulation: $A$ new and promising therapeutic approach for chronic heart failure Eur Heart J, 32(7), p. 847-55.

4. Del Rio, C.L., T.A. Dawson, B.D. Clymer, D.J. Paterson, and G.E. Billman (2008) Effects of acute vagal nerve stimulation on the early passive electrical changes induced by myocardial ischaemia in dogs: Heart rate-mediated attenuation Exp Physiol, 93(8), p. 931-44.

5. Grimm, S. and M. Bajbouj (2010) Efficacy of vagus nerve stimulation in the treatment of depression Expert Rev Neurother, 10(1), p. 87-92.

6. Merkel, F.K., A.M. Bilgutay, and W. Lillehei (1968) Vagal tuning for angina pectoris Journal of the American Medical Association, 204(6), p. 529.

7. Schwartz, P.J. and G.M. De Ferrari (2009) Vagal stimulation for heart failure: Background and first in-man study Heart Rhythm, 6(11), p. S76-S81.

8. Schwartz, P.J., G.M. De Ferrari, A. Sanzo, M. Landolina, R. Rordorf, C. Raineri, C. Campana, M. Revera, N. Ajmone-Marsan, L. Tavazzi, and A. Odero (2008) Long term vagal stimulation in patients with advanced heart failure: First experience in man Eur J Heart Fail, 10(9), p. 884-91. 
9. Vanoli, E., G.M. De Ferrari, M. Stramba-Badiale, S.S. Hull, Jr., R.D. Foreman, and P.J. Schwartz (1991) Vagal stimulation and prevention of sudden death in conscious dogs with a healed myocardial infarction Circ Res, 68(5), p. 1471-81.

10. Zhang, Y. and T.N. Mazgalev (2011) Arrhythmias and vagus nerve stimulation Heart Fail Rev, 16(2), p. 147-61.

11. Zhang, Y.H., Z.B. Popovic, S. Bibevski, I. Fakhry, D.A. Sica, D.R. Van Wagoner, and T.N. Mazgalev (2009) Chronic vagus nerve stimulation improves autonomic control and attenuates systemic inflammation and heart failure progression in a canine high-rate pacing model Circulation-Heart Failure, 2(6), p. 692-9.

12. Levy, M.N. (1984) Cardiac sympathetic-parasympathetic interactions Fed Proc, 43(11), p. 2598-602.

13. Levy, M.N. (1971) Sympathetic-parasympathetic interactions in the heart Circ Res, 29(5), p. 437-45.

14. Kollai, M. and K. Koizumi (1979) Reciprocal and non-reciprocal action of the vagal and sympathetic-nerves innervating the heart $\mathrm{J}$ Autonom Nerv Syst, 1(1), p. 33-52.

15. Kollai, M. and K. Koizumi (1981) Cardiovascular reflexes and interrelationships between sympathetic and parasympathetic activity $\mathrm{J}$ Autonom Nerv Syst, 4(2), p. 135-48.

16. Martini, F.H., Fundamentals of anatomy and physiology. 8th ed. 2006: Prentice Hall.

17. Boron, W.F. and E.L. Boulpaep, Medical physiology. 1st ed. 2003: Saunders.

18. Gray, H., R. Warwick, P. Williams, M. Dyson, L. Bannister, and R. Moore, Gray's anatomy. 1980: Churchill Livingstone.

19. Armour, J.A., D.A. Murphy, B.X. Yuan, S. Macdonald, and D.A. Hopkins (1997) Gross and microscopic anatomy of the human intrinsic cardiac nervous system Anat Rec, 247(2), p. 289-98.

20. Pauza, D.H., V. Skripka, and N. Pauziene (2002) Morphology of the intrinsic cardiac nervous system in the dog: A whole-mount study employing histochemical staining with acetylcholinesterase Cells Tissues Organs, 172(4), p. 297-320.

21. Pauza, D.H., V. Skripka, N. Pauziene, and R. Stropus (2000) Morphology, distribution, and variability of the epicardiac neural ganglionated subplexuses in the human heart Anat Rec, 259(4), p. 35382.

22. Yuan, B.X., J.L. Ardell, D.A. Hopkins, A.M. Losier, and J.A. Armour (1994) Gross and microscopic anatomy of the canine intrinsic cardiac nervous system Anat Rec, 239(1), p. 75-87.

23. Armour, J.A. (2007) The little brain on the heart Cleve Clin J Med, 74 Suppl 1, p. S48-51. 
24. Armour, J.A. and J.L. Ardell, Basic and clinical neurocardiology. 2004: Oxford University Press, USA.

25. Arndt, J.O., A. Dorrenhaus, and H. Wiecken (1975) The aortic arch baroreceptor response to static and dynamic stretches in an isolated aorta-depressor nerve preparation of cats in vitro J Physiol, 252(1), p. 59-78.

26. Zucker, I.H. and J.P. Gilmore (1973) Left atrial receptor discharge during atrial arrhythmias in the dog Circ Res, 33(6), p. 672-7.

27. Hakumaki, M.O. (1987) Seventy years of the bainbridge reflex Acta Physiol Scand, 130(2), p. 177-85.

28. Davidson, N.S., S. Goldner, and D.I. Mccloskey (1976) Respiratory modulation of baroreceptor and chemoreceptor reflexes affecting heartrate and cardiac vagal efferent nerve activity J Physiol-London, 259(2), p. 523-30.

29. Campagna, J.A. and C. Carter (2003) Clinical relevance of the bezoldjarisch reflex Anesthesiology, 98(5), p. 1250-60.

30. Armour, J.A. (2008) Potential clinical relevance of the 'little brain' on the mammalian heart Exp Physiol, 93(2), p. 165-76.

31. Furness, J.B. (2000) Types of neurons in the enteric nervous system J Autonom Nerv Syst, 81(1-3), p. 87-96.

32. Goyal, R.K. and I. Hirano (1996) Mechanisms of disease - the enteric nervous system New Eng J Med, 334(17), p. 1106-15.

33. Wine, J.J. (2007) Parasympathetic control of airway submucosal glands: Central reflexes and the airway intrinsic nervous system Autonomic Neuroscience-Basic \& Clinical, 133(1), p. 35-54.

34. Olson, T.R. and W. Pawlina, A.D.A.M. Student atlas of anatomy. 2nd ed. 2008, New York: Cambridge University Press.

35. Arndt, J.O., P. Brambring, K. Hindorf, and M. Rohnelt (1971) Afferent impulse traffic from atrial a-type receptors in cats - does a-type receptor signal heart rate Pflug Arch Eur J Phy, 326(4), p. 300-15.

36. Coleridge, H.M., C. Kidd, and J.C.G. Coleridge (1964) Cardiac receptors in dog with particular reference to 2 types of afferent ending in ventricular wall J Physiol-London, 174(3), p. 323-39.

37. Gilmore, J.P. and I.H. Zucker (1974) Discharge of type-b atrial receptors during changes in vascular volume and depression of atrial contractility J Physiol-London, 239(2), p. 207-23.

38. Hakumaki, M.O. (1972) Vagal and sympathetic efferent discharge in bainbridge reflex of dogs Acta Physiol Scand, 85(3), p. 414-7.

39. Iriuchijima, J. and M. Kumada (1964) Activity of single vagal fibres efferent to heart Japanese Journal of Physiology, 14(5), p. 479-87.

40. Jewett, D.L. (1964) Activity of single efferent fibres in cervical vagus nerve of dog with special reference to possible cardio-inhibitory fibres $\mathbf{J}$ Physiol-London, 175(3), p. 321-57. 
41. Kollai, M., K. Koizumi, H. Yamashita, and C.M. Brooks (1978) Study of cardiac sympathetic and vagal efferent activity during reflex responses produced by stretch of atria Brain Res, 150(3), p. 519-32.

42. Middleton, S., H.H. Middleton, and H. Grundfest (1950) Spike potentials and cardiac effects of mammalian vagus nerve Am J Physiol, 162(3), p. 545-52.

43. Neil, E. and N. Joels (1961) Impulse activity in cardiac afferent vagal fibres N-S Arch Ex Path Pharmakol, 240(5), p. 453-60.

44. Paintal, A.S. (1953) A study of right and left atrial receptors J PhysiolLondon, 120(4), p. 596-610.

45. Sleight, P. and Widdicombe (1965) Action potentials in afferent fibres from pericardial mechanoreceptors in dogs J Physiol-London, 181(2), p. 259-69.

46. Thoren, P.N. (1976) Activation of left ventricular receptors with nonmedullated vagal afferent fibres during occlusion of a coronary artery in the cat Am J Cardiol, 37(7), p. 1046-51.

47. Whitteridge, D. (1948) Afferent nerve fibres from the heart and lungs in the cervical vagus J Physiol-London, 107(4), p. 496-512.

48. Kandel, E.R., J.H. Schwartz, and T.M. Jessell, Principles of neuroscience. 4th ed. Principles of neural science. 2000: McGraw-Hill.

49. Blair, E. and J. Erlanger (1933) A comparison of the characteristics of axons through their individual electrical responses Am J Physiol, 106, p. 524-64.

50. Agostoni, E., J.E. Chinnock, M.B. De Daly, and J.G. Murray (1957) Functional and histological studies of the vagus nerve and its branches to the heart, lungs and abdominal viscera in the cat $\mathrm{J}$ Physiol, 135(1), p. 182-205.

51. Cheng, Z.X. and T.L. Powley (2000) Nucleus ambiguus projections to cardiac ganglia of rat atria: An anterograde tracing study J Comp Neurol, 424(4), p. 588-606.

52. Evans, D.H.L. and J.G. Murray (1954) Histological and functional studies on the fibre composition of the vagus nerve of the rabbit $\mathrm{J}$ Anat, 88(3), p. 320-37.

53. Ford, T.W. and P.N. Mcwilliam (1986) The effects of electricalstimulation of myelinated and nonmyelinated vagal fibres on heart-rate in the rabbit J Physiol-London, 380(Nov), p. 341-7.

54. Jones, J.F.X., Y. Wang, and D. Jordan (1995) Heart-rate responses to selective stimulation of cardiac vagal-c fibres in anesthetized cats, rats and rabbits J Physiol-London, 489(1), p. 203-14.

55. Nielsen, K.R., C. Sevcencu, and J.J. Struijk. Vagus nerve activity based prediction of epileptic seizures in rats. in 13th Annual Conference of the IFESS. 2008. Freiburg, Germany.

56. Rozman, J. and S. Ribaric (2007) Selective recording of electroneurograms from the left vagus nerve of a dog during 
stimulation of cardiovascular or respiratory systems Chinese J Physiol, 50(5), p. 240-50.

57. Yambe, T., S. Nanka, S. Kobayashi, A. Tanaka, M. Yoshizawa, T. Abe, K. Tabayashi, H. Takeda, and S. Nitta (1999) Vagal nerve activity recording in the awake condition for the control of an artificial heart system Artif Organs, 23(6), p. 529-31.

58. Ogawa, M., S.M. Zhou, A.Y. Tan, J. Song, G. Gholmieh, M.C. Fishbein, H. Luo, R.J. Siegel, H.S. Karagueuzian, L.S. Chen, S.F. Lin, and P.S. Chen (2007) Left stellate ganglion and vagal nerve activity and cardiac arrhythmias in ambulatory dogs with pacing-induced congestive heart failure J Am Coll Cardiol, 50(4), p. 335-43.

59. Olshansky, B., H.N. Sabbah, P.J. Hauptman, and W.S. Colucci (2008) Parasympathetic nervous system and heart failure: Pathophysiology and potential implications for therapy Circulation, 118(8), p. 863-71.

60. Arora, R.C., R. Cardinal, F.M. Smith, J.L. Ardell, L.J. Dell'Italia, and J.A. Armour (2003) Intrinsic cardiac nervous system in tachycardia induced heart failure Am J Physiol Regul Integr Comp Physiol, 285(5), p. R1212-23.

61. Bibevski, S. and M.E. Dunlap (2011) Evidence for impaired vagus nerve activity in heart failure Heart Fail Rev, 16(2), p. 129-35.

62. Kinugawa, T. and M.E. Dibner-Dunlap (1995) Altered vagal and sympathetic control of heart rate in left ventricular dysfunction and heart failure Am J Physiol, 268(2 Pt 2), p. R317-23.

63. La Rovere, M.T., J.T. Bigger, Jr., F.I. Marcus, A. Mortara, and P.J. Schwartz (1998) Baroreflex sensitivity and heart-rate variability in prediction of total cardiac mortality after myocardial infarction. Atrami (autonomic tone and reflexes after myocardial infarction) investigators Lancet, 351(9101), p. 478-84.

64. Lechat, P., J.S. Hulot, S. Escolano, A. Mallet, A. Leizorovicz, M. Werhlen-Grandjean, G. Pochmalicki, and H. Dargie (2001) Heart rate and cardiac rhythm relationships with bisoprolol benefit in chronic heart failure in cibis ii trial Circulation, 103(10), p. 1428-33.

65. Bibevski, S. and M.E. Dunlap (2004) Prevention of diminished parasympathetic control of the heart in experimental heart failure Am J Physiol Heart Circ Physiol, 287(4), p. H1780-5.

66. Leon, H., J.C. Guzman, T. Kuusela, R. Dillenburg, M. Kamath, and C.A. Morillo (2005) Impaired baroreflex gain in patients with inappropriate sinus tachycardia J Cardiovasc Electr, 16(1), p. 64-8.

67. Inagaki, M., T. Kawada, M. Lie, C. Zheng, K. Sunagawa, and M. Sugimachi (2005) Intravascular parasympathetic cardiac nerve stimulation prevents ventricular arrhythmias during acute myocardial ischemia Conf Proc IEEE Eng Med Biol Soc, 7, p. 7076-9. 
68. Zuanetti, G., G.M. De Ferrari, S.G. Priori, and P.J. Schwartz (1987) Protective effect of vagal stimulation on reperfusion arrhythmias in cats Circ Res, 61(3), p. 429-35.

69. Springer, J., D.O. Okonko, and S.D. Anker (2004) Vagal nerve stimulation in chronic heart failure: An antiinflammatory intervention? Circulation, 110(4), p. e34

70. Tracey, K.J. (2002) The inflammatory reflex Nature, 420(6917), p. 8539. 


\title{
Chapter 2
}

Neural signals modulated with the cardiac cycle can be measured extraneurally from the cervical vagus nerve

\author{
Simone Ordelman \\ Lilian Kornet \\ Richard Cornelussen \\ Rik Buschman \\ Peter Veltink
}

Submitted 


\section{Abstract}

The vagus nerve conducts signals emanating from cardiac receptors, directed to the brain. These signals may be useful in monitoring cardiovascular pathological conditions. Extraneural recording of these signals is challenging, because of the large number of non-cardiac signals that are conducted by the vagus nerve. In this chapter we address sensing the cardiac-modulated signals from the cervical vagus nerve with an extraneural cuff electrode. We compare the use of a cuff with rings to a cuff with multiple contacts, the latter hypothesized to be more effective in sensing the cardiac signals.

In fourteen out of fifteen pigs a significant cardiac-modulated pattern was found. A multi-contact cuff was more effective in recording these signals than a cuff with ring electrodes. Cardiac-modulated signals were recorded in $90 \%$ versus $60 \%$ of the cases respectively. The phase of the cardiac cycle in which cardiac-modulated signals dominated changed from the QRST and TP phase to the PQ and QRST phase when blood pressure and ventricular contractility were increased due to dobutamine administration. We conclude that signals originating from cardiac receptors can be recorded extraneurally on the vagal trunk. This may be useful for chronic monitoring and subsequent clinical intervention. Average reference recording increases the odds of sensing a cardiac-modulated pattern. 


\subsection{Introduction}

The status of the heart is communicated via afferent vagal and sympathetic fibres to the central nervous system. The signals originate from receptors that are sensitive to regional mechanical events, chemical events, or both.

Many of the cardiac mechanoreceptors with vagal afferents fire in phase with the cardiac cycle. The signals transduced by these vagal afferent fibres have been studied from single-fibre recordings [1-6]. Atrial receptors with myelinated vagal afferents have been found to fire during atrial contraction (type A) or atrial filling (type B) [2]. Type A atrial receptors are possibly influenced by contraction of the heart [1], whereas type B atrial receptors are sensitive to changes in blood volume and central venous pressure [4]. Ventricular receptors with myelinated vagal afferents discharge at the onset of ventricular contraction [5]. They respond to rise in intra-ventricular pressure [5]. Coronary artery receptors with myelinated vagal afferents show a cardiacmodulated rhythm in phase with coronary pressure [3]. Receptors with unmyelinated afferents have also been found, but only the receptors located in the atria sometimes exhibit a cardiac-modulated rhythm, either in phase with atrial contraction or atrial filling [6]. Cardiac chemoreceptors do not show patterns modulated with the cardiac cycle [4].

The firing patterns described above were obtained from single-fibre recordings. Obtaining these recordings requires dissection of individual fibres in the nerve and is therefore not suited for chronic clinical application. Extraneural electrodes around the cervical vagus nerve have been used clinically for electrical stimulation to treat epilepsy [7]. Signals recorded by such an extraneural electrode may be used to monitor arrhythmias or other pathological conditions that have been shown to have a relation with vagal activity, such as heart failure [8] or ischemia [9-10].

The challenge of extraneural recording lies in detecting the relevant, cardiac signals from the large amount of activity transmitted through the vagus nerve. Cardiac vagal fibres are spatially concentrated in one or multiple fascicles [1112]. About ten percent of all fibres in the cervical vagus nerve are cardiac fibres [11]. Approximately 80 percent of those cardiac fibres are afferent [11]. The signals of atrial, ventricular and coronary artery receptors are conducted by myelinated fibres, and signals from myelinated fibres have a relatively high amplitude compared to unmyelinated fibres [13]. However, their activity will be obscured by the large number of other large myelinated fibres in the cervical vagus nerve, such as laryngeal and bronchial fibres [11].

In the current study, we investigate the feasibility of recording cardiac modulated signals in pigs using an extraneural electrode cuff placed around the vagal trunk. The pig is chosen, because it has a neurocardiac system similar to that of humans in anatomy and physiology [14-17]. 
We assume to improve effectiveness in recording from cardiac fibres by using a multi-contact electrode configuration over a ring electrode configuration. Multicontact electrode configurations have been shown to improve spatial recording selectivity in peripheral nerves [18]. In addition it is expected that the dominant phase in the cardiac cycle of the cardiac-modulated signal will change when blood pressure and ventricular contractility are increased by dobutamine, due to changes in cardiac receptor firing.

\subsection{Methods}

\subsubsection{Ethical approval}

All experiments were approved by the Ethics Committee on Experimental Animal Welfare of the University of Maastricht.

\subsubsection{Experimental setup}

Experiments were performed in fifteen female Dutch Landrace pigs. Animal handling was performed according to the Dutch Law and the European Directive for the Protection of Animals and was approved by the Experimental Animal Committee of the Maastricht University. The pig was premedicated with $0.5 \mathrm{mg} / \mathrm{kg}$ Morphine 20 minutes before induction with Zoletil (Telazol), $3 \mathrm{mg} / \mathrm{kg}$ and xylazine $0.5 \mathrm{mg} / \mathrm{kg}$. The pig was anesthetized with a combination of isoflurane $(1-1.5 \%)$ and fentanyl $(6-10 \mu \mathrm{g} / \mathrm{kg} / \mathrm{hr})$. At the end of the experiment, the pig was euthanized with an overdose of pentobarbital. The left vagus nerve was carefully dissected free from the surrounding tissue at the cervical level and a custom-made self-coiling cylindrical cuff electrode configuration was placed. The cuff was $15 \mathrm{~mm}$ long and had three circular Pt/Ir electrode contacts with an inter-electrode distance of $4 \mathrm{~mm}$ (figure 2.1a). In ten pigs, an additional cuff was placed that had 12 contacts instead of three rings (figure 2.1b). This second cuff was placed a few mm cranial to the first cuff. The cuffs were made in various diameters $(2,3.5$ and $5 \mathrm{~mm})$, to ensure a proper fit around the nerve. Variations in nerve diameter can also be accommodated by the flexibility in tightness of the cuff's coiling. Surface electrodes were placed on shaved paws for recording ECG. Left ventricular pressure was measured with a Millar catheter (Millar Instruments, Houston, Texas, USA).
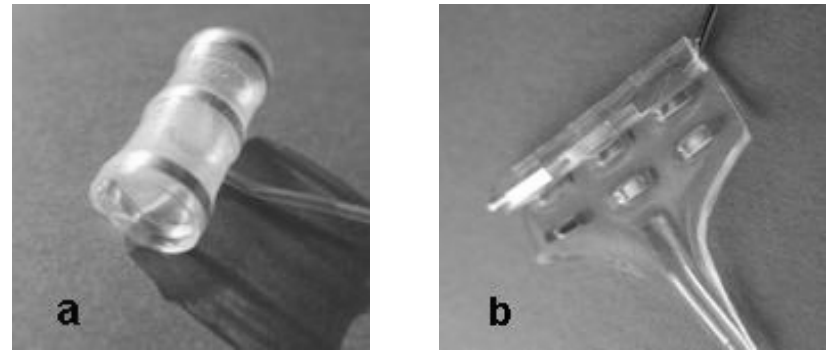

Figure 2.1: a) Electrode cuff with three rings. b) Electrode cuff with 12 contacts 
ENG signals were amplified by a TMSi Refa amplifier (TMS international, Oldenzaal, The Netherlands). ECG and blood pressure recordings were amplified by a TMSi Porti amplifier (TMS international, Oldenzaal, The Netherlands). ENG (Electro Neuro Gram, which is the recorded electrical neural activity) was sampled at a frequency of $20 \mathrm{kHz}$ and the ECG and blood pressure recordings were sampled at $2048 \mathrm{~Hz}$.

All data were stored on a laptop using PortiLab2 software (TMS international, Oldenzaal, The Netherlands). Signals were measured using the average reference method [19-20], which uses the average of all signals as a reference. It allows separate recording of ENG signals from all electrode sites on a cuff, which may improve the odds of recording from a subpopulation of cardiac vagal fibres. Subtracting the average of all signals also helps to reduce noise.

Baseline recordings of neural activity, blood pressure and ECG were made with a duration of five minutes. In seven pigs, dobutamine was administered in order to change the state of the cardiovascular system and the five minute recording was repeated. Dobutamine (a sympathomimetic drug) increases heart rate, blood pressure and left-ventricular contractility by stimulating the $\beta 1$ receptors on the heart.

\subsubsection{Signal analysis}

Offline signal analysis was performed in Matlab R2007a (The Mathworks Inc.). R-tops of the ECG were detected automatically and confirmed by visual inspection. Left ventricular contractility for each cardiac cycle was assessed using maximum values of the time derivative of left ventricular pressure: $\left(\frac{\mathrm{dP}_{\mathrm{LV}}}{\mathrm{dt}}\right)_{\max }$ [21]. This parameter will be called left ventricular contractility (LVC). The value of systolic left ventricular pressure $\left(\mathrm{P}_{\mathrm{LV}}\right)$ for each cardiac cycle was determined as the peak pressure of that cycle.

Neural recordings can be contaminated by EMG signals (Electro Myo Gram, which is the recorded electrical muscular activity) from sources outside the cuff [22-23]. The frequency content of EMG ranges from $1 \mathrm{~Hz}$ to $3 \mathrm{kHz}$, with a peak at approximately $250 \mathrm{~Hz}$. The frequency range of neural signals is $1 \mathrm{kHz}$ to 10 $\mathrm{kHz}$, and maximal power is below $3 \mathrm{kHz}$ [24]. Signal to noise ratio (SNR) was estimated before and after the signal was filtered, by dividing the mean power of the raw signal in the ENG frequency band $(1 \mathrm{kHz}$ to $10 \mathrm{kHz})$ by the mean power of the raw signal in the EMG frequency band (1 Hz to $1 \mathrm{kHz})$. The EMG frequency band is cut off at $1 \mathrm{kHz}$ instead of $3 \mathrm{kHz}$, to avoid overlap between the EMG and ENG frequency band in the SNR estimation. It should be noted that EMG peak activity is around $250 \mathrm{~Hz}$ and ENG peak activity is below $3 \mathrm{kHz}$. Filtering and ensemble averaging of the signals should reduce the EMG interference and improve SNR. 
The centre ring electrode has the same derivation as a tripolar configuration, except for a factor of 1.5 :

$$
\begin{aligned}
& \mathrm{S}_{\mathrm{ar}, 2}=\mathrm{s}_{2}-\frac{1}{3} \sum_{\mathrm{i}=1}^{3} \mathrm{~s}_{\mathrm{i}}=\frac{2}{3} \mathrm{~s}_{2}-\frac{1}{3} \mathrm{~s}_{1}-\frac{1}{3} \mathrm{~s}_{3} \\
& \mathrm{~S}_{\text {trip }}=\mathrm{s}_{2}-\frac{1}{2} \mathrm{~s}_{1}-\frac{1}{2} \mathrm{~s}_{3}=1.5 \mathrm{~s}_{\mathrm{ar}, 2}
\end{aligned}
$$

With $\mathrm{s}_{\mathrm{i}}$ the input signals, $\mathrm{S}_{\mathrm{ar}, 2}$ the recorded signal on the centre ring electrode with the average reference method, and $\mathrm{S}_{\text {trip }}$ the recorded signal with the tripolar derivation. Since a tripolar configuration is known to reduce EMG interference $[22,25]$, the signal recorded on the centre ring electrode is expected to have a higher SNR before filtering than a signal recorded on an outer ring electrode if using the average reference configuration. To test this, the estimated SNR of the centre ring electrode is compared to the estimated SNR of the outer ring electrodes.

ENG was band pass filtered between $800 \mathrm{~Hz}$ and $9000 \mathrm{~Hz}$ with a second order Butterworth filter. The cut-off frequency of $800 \mathrm{~Hz}$ retains the signal that fire in phase with the cardiac cycle and reduces ECG artefacts [26] and EMG interference [24]. The low-pass cut off frequency of $9000 \mathrm{~Hz}$ is applied to improve signal-to-noise ratio by reducing high-frequency noise from the electrodes or the amplifier. Subsequently, the signal was rectified and low-pass filtered at $10 \mathrm{~Hz}$, resulting in the envelope of the ENG. The envelope of the ENG was obtained to inspect variations and patterns in vagal activity. For each pig, an ensemble average was made from the ENG of each recording electrode. For each of these ensemble averages, 260 data segments were used, with a length equal to that recording's average cardiac cycle length, and centred on Rtops of the ECG. 260 Segments were used, because this was the maximum number that could be obtained from the five minutes recordings of all pigs. An ensemble average of 65,130 , and 195 cycles was also inspected to obtain an indication of how many cycles are minimally needed. A smaller time window will allow observation of more rapid fluctuations or changes. Mean activity $\left(\mathrm{V}_{\mathrm{avr}}\right)$ was subtracted to obtain only the variations within a cardiac cycle. Remaining variations ascribed to stochastic noise were reduced by ensemble averaging. The 95\%-confidence interval of the ensemble averaged signals was inspected. If this interval was above zero at any point during the cardiac cycle, it was concluded that these variations in the signal were not due to noise. These events were classified as significant increases of neural activity. Significant decreases of neural activity were not analyzed. Since the mean activity was subtracted, we assume these decreases to be phases of little to no cardiacmodulated activity, which provide no physiologically relevant information for our purpose. The recording on the electrode which showed the most significant modulation was selected for each pig and used for analyses. 
The shape of the pattern was described by the phases of a cardiac cycle in which the signal showed a peak that was significantly increased compared to the average of the cardiac cycle. The phase in which the activity was increased can be used to identify the population of receptors that this activity originated from. The three phases that were distinguished were the PQ phase (from the beginning of the $\mathrm{P}$ wave to the beginning of the $\mathrm{Q}$ wave), the QRST phase (from the beginning of the $\mathrm{Q}$ wave to the beginning of the $\mathrm{T}$ wave) and the TP phase (from the beginning of the $\mathrm{T}$ wave to the beginning of the $\mathrm{P}$ wave). Steepest initial slopes were used to identify the beginning of waves. The PQ phase coincides with atrial contraction, the QRST phase with ventricular contraction and the TP phase with diastole.

Additionally, for each pig the neural activity level was quantified by the mean voltage of the ensemble averaged signal $\left(\mathrm{V}_{\text {avr }}\right)$. The changes in $\mathrm{V}_{\mathrm{avr}}, \mathrm{P}_{\mathrm{LV}}$ and LVC with increased blood pressure and ventricular contractility caused by dobutamine were normalized to the baseline values of the same pig, to enable averaging across pigs and to emphasize the effect of increased blood pressure and ventricular contractility.

Statistical analysis was performed in SPSS software (SPSS, version 12.0.1, SPSS Inc., Chicago, Illinois, USA). All collected data were tested for normality with a Shapiro-Wilk test. Data are represented as mean $\pm \mathrm{SD}$. Values of $\mathrm{V}_{\text {avr }}$ were compared between baseline and dobutamine condition with a Student's paired t-test for normally distributed data and a Wilcoxon Signed Rank test otherwise.

\subsection{Results}

The filtering process of the neural signals is depicted in figure 2.2. An example of a raw signal is shown. The raw signals are in the range of a few $\mu \mathrm{V}$, which is typical for neural recordings. The subsequent panels show the signal after the filtering steps are completed: a band-pass filter between $800 \mathrm{~Hz}$ and $9000 \mathrm{~Hz}$, rectification, and a $10 \mathrm{~Hz}$ low-pass filter to obtain the envelope of the signal. The envelope of the signal is used to analyze the activity patterns in the ensemble averaging. The ensemble average of this signal is shown in figure 2.3. Figure 2.3 shows that the cardiac-modulated pattern may not be apparent from vagal activity in an individual cardiac cycle. Estimated SNR expressed as median (interquartile range) improves when the signal is bandpass filtered, from $1.1 * 10^{-4}\left(3.2^{*} 10^{-5}\right)$ to $2.7(2.6)(\mathrm{N}=15)$. Looking at the ring electrodes only, the estimated SNR before filtering of the centre electrode is $1.1^{*} 10^{-4}\left(2.3^{*} 10^{-5}\right)$ and of the outer electrodes it is $1.2 * 10^{-4}\left(4.3 * 10^{-5}\right)$. After filtering the centre electrode has an estimated SNR of 2.3 (2.1) and the outer electrodes have an estimated SNR of $2.5(1.2)$. 
In fourteen out of fifteen pigs a significant cardiac-modulated pattern was found in the vagal signal in baseline, in nine out of fifteen pigs using the ring cuff $(60 \%)$, and in nine out of ten pigs (90\%) using the multi-contact cuff. With the ring cuff, in two out of nine pigs a cardiac-modulated pattern was observed on both the outer and centre rings. In the other seven pigs, the pattern was only found on one of the outer rings. With the multi-contact cuff, the modulation was always seen on two or more contacts of the multi-contact cuff.

For the fourteen pigs in which a significant cardiac-modulated pattern was found during baseline, using either the ring cuff or the multi-contact cuff, the phases of increased activity were analyzed from the electrode with the most significant pattern. During baseline condition, significantly increased neural activity compared to the average of the cardiac cycle, was found in the PQ phase $(n=1)$, the QRST phase $(n=5)$ and/or the TP phase $(n=10)$. Table 2.1 shows the phases of significantly increased activity per pig.

Table 2.1: The phase of significantly increased vagal activity per pig. Shaded areas indicate when no data is available.

\begin{tabular}{l|l|l|l|l|l|l} 
& \multicolumn{3}{|c|}{ Baseline } & \multicolumn{3}{c}{ Dobutamine } \\
\hline Pig & PQ & QRST & TP & PQ & QRST & TP \\
\hline 1 & & $\mathrm{x}$ & $\mathrm{x}$ & & $\mathrm{x}$ & \\
\hline 2 & & & $\mathrm{x}$ & & $\mathrm{x}$ & \\
\hline 3 & & $\mathrm{x}$ & & $\mathrm{x}$ & & \\
\hline 4 & & & $\mathrm{x}$ & & $\mathrm{x}$ & \\
\hline 5 & & & $\mathrm{x}$ & & & \\
\hline 6 & & & $\mathrm{x}$ & & & \\
\hline 7 & & $\mathrm{x}$ & $\mathrm{x}$ & & & \\
\hline 8 & & & & & & \\
\hline 9 & & & $\mathrm{x}$ & & & \\
\hline 10 & & & $\mathrm{x}$ & & & \\
\hline 11 & & & $\mathrm{x}$ & $\mathrm{x}$ & & \\
\hline 12 & & & $\mathrm{x}$ & & & \\
\hline 13 & & $\mathrm{x}$ & & & & \\
\hline 14 & & $\mathrm{x}$ & & & & \\
\hline 15 & $\mathrm{x}$ & & & & & \\
\hline Total & 1 & 5 & 10 & 2 & 3 & 0
\end{tabular}



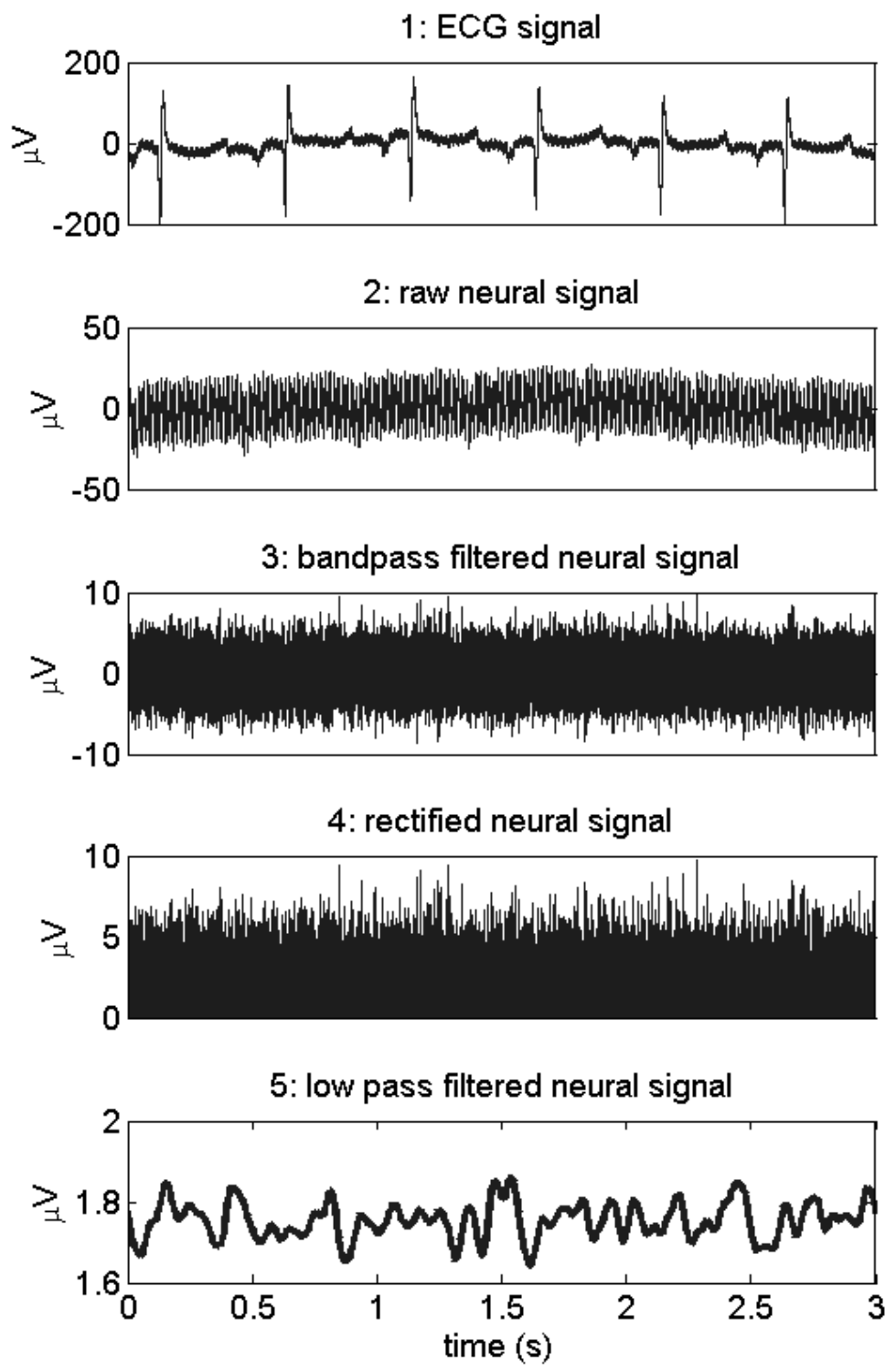

Figure 2.2: Filtering process of a signal from an outer ring electrode. 1: the ECG signal. 2: the raw signal. 3: the raw signal is bandpass filtered between $800 \mathrm{~Hz}$ and $9000 \mathrm{~Hz}$. This filter retains the neural signal and reduces ECG artefacts and EMG interference. 4: the signal is then rectified.5: the signal is low pass filtered at $10 \mathrm{~Hz}$ to obtain the envelope of the signal. 

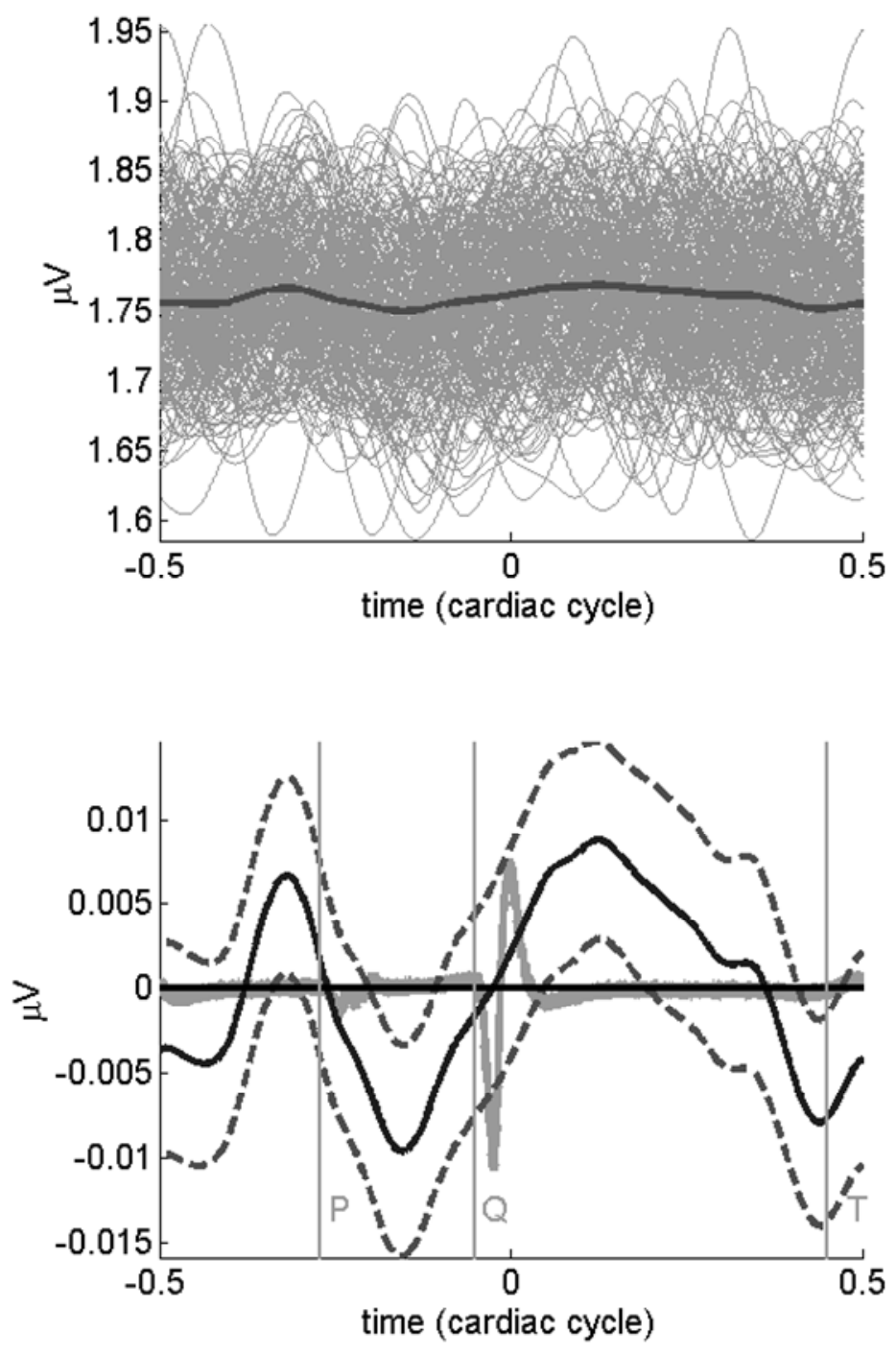

Figure 2.3: Example of a significant vagal activity pattern in the cardiac cycle from an outer ring electrode (the same signal as in figure 2.2). Activity is increased during the TP phase and the QRST phase in this example. The top frame shows the individual signal segments from the envelope of the signal in grey and the resulting ensemble average in black. The bottom frame shows the ensemble averaged activity (black solid line) aligned with the $R$ top of the ECG (grey solid line). The dashed lines indicate the 95\% confidence interval. For reference, the average ECG is plotted. Vertical lines indicate the beginning of the $P$-wave, $Q$-wave and T-wave respectively. 
The number of cycles that was needed in the ensemble average to obtain a significantly cardiac-modulated pattern varied between pigs. In four out of fourteen pigs 65 cycles sufficed, in seven pigs 130 cycles sufficed, in one pig 195 cycles sufficed and in two pigs all 260 cycles were needed to result in a significantly cardiac-modulated signal. There was no relationship found between the cardiac phase of increased activity and the number of cycles needed for a significant modulation.

In the seven pigs in which measurements were performed after dobutamine was administered, LVC was $73 \pm 22 \%$ higher, and $\mathrm{P}_{\mathrm{LV}}$ was $31 \pm 21 \%$ higher than during baseline recordings, which were significant increases.

In these recordings, a significant cardiac-modulated pattern was found in five pigs. Increased activity compared to the average of the cardiac cycle was observed, using either the ring cuff or the multi-contact cuff, during the PQ phase $(n=2)$ or the QRST phase $(n=3)$ and never during the TP phase. Despite the increase in blood pressure and ventricular contractility with dobutamine, no statistically significant effect on $\mathrm{V}_{\text {avr }}$ was found.

\subsection{Discussion}

This study is the first to sense vagal activity patterns that are modulated with the cardiac cycle using an extraneural electrode cuff in pigs. We were able to record such patterns in fourteen out of fifteen pigs. Possibly in the other pig, noise levels were too high or the cardiac fibres were located too far away from the perimeter.

During baseline condition, the cardiac-modulated pattern showed peaks of significantly increased neural activity compared to the average of the cardiac cycle in all three phases of the ECG; the PQ phase $(n=1)$, the QRST phase $(n=5)$ and/or the TP phase $(n=10)$. Thus, neural activity is mostly concentrated in the TP phases during baseline condition. It was expected that cardiac activity would be found in all phases of the cardiac cycle, since the cervical vagus nerve comprises afferent cardiac fibres that fire during atrial systole, ventricular systole, diastole or multiple phases.

For example, atrial receptors with myelinated afferents, firing during atrial contraction (type A) and atrial filling (type B) [2], may account for the peaks of increased activity concentrated in the PQ phase and the TP phase respectively. Ventricular [5] and coronary [3] mechanoreceptors with myelinated vagal afferents, discharging during systole, may account for the increased activity in the QRST phase.

Phases with increased activity in the sensed signals may depend on the position of the associated afferent fibres relative to the electrodes in the individual pigs. This may differ, due to inter-individual neural anatomy differences. It is unknown if this was the case, since no histological studies were performed on 
the nerves recorded from. The difference in diameter between the cardiac afferents is small [27] and is therefore an unlikely cause of the differences in the recorded cardiac-modulated patterns.

The number of cycles that is needed in the ensemble average to obtain a significantly cardiac-modulated pattern varied between pigs, which means that the time frame in which a change in the pattern will manifest itself will also differ between pigs. Since there was no relationship found between the cardiac phase of increased activity and the number of cycles needed for a significant modulation, it can be concluded that the type of receptor that creates the pattern is not a contributing factor to the significance of the pattern. Factors such as electrode-nerve contact, noise, EMG interference, and position of cardiac fascicles in the nerve likely determine how many cycles are needed to reveal the cardiac-modulated pattern.

As hypothesized, the cardiac-modulated pattern in recordings when blood pressure and ventricular contractility increased with dobutamine as found in 5 out of 7 pigs had a different shape compared to baseline recordings in all five pigs. Dobutamine is a sympathomimetic drug that increases heart rate and leftventricular contractility and pressure by stimulating the $\beta 1$ receptors on the heart. Phases in which peaks of increased activity compared to the average of the cardiac cycle were observed were the PQ phase $(n=2)$ and the QRST phase $(n=3)$ and never the TP phase (table 2.1). This shows that cardiac-modulated neural activity is concentrated around atrial and ventricular contraction when blood pressure and ventricular contractility were increased by dobutamine.

This concentrated activity during systole may be explained by a relative increase in firing rate from type A atrial, ventricular and coronary mechanoreceptors with respect to firing rate of type B atrial receptors. Type B atrial receptors, which fire in the TP phase, respond to changes in total blood volume, central volume and central venous pressure. Dobutamine does not generally affect these parameters [28]. In contrast, type A atrial receptors are influenced by contraction of the heart [1] and therefore the increased activity in the PQ phase may be linked to the increase in heart rate brought about by dobutamine. Ventricular receptors respond to ventricular pressure and contractility $[5,29]$. The increased activity in the QRST phase may therefore be linked to the increased pressure and contractility when dobutamine was administered. Coronary mechanoreceptors may have increased their firing rate due to an increased coronary pressure, which may also explain the increased activity in the QRST phase.

It was found that by using a multi-contact electrode cuff the cardiac modulated signal was sensed more readily compared to a cuff with rings, on a selection of the contacts, most likely by improving selective recording from the cardiac fibres. A cardiac-modulated signal was found in nine out of fifteen pigs (60\%) on the ring cuff and in nine out of ten pigs $(90 \%)$ on the multi-contact cuff. 
On the multi-contact electrode cuff a pattern was seen on multiple contacts. The contacts that showed the cardiac-modulated signal were likely located closest to the cardiac fibres. Fibre histology should be included in future studies to verify the link between fibre type and the signals recorded.

Cardiac-modulated signals as described in this chapter have been analyzed in rats for their relationship to epileptic seizures [26]. Spectrograms of vagal activity showed peak activity mostly in the PQ and QRST phase. During an epileptic seizure, a shift in the peak activity was observed to the time shortly after the $\mathrm{S}$ phase. This harmonizes with our findings, in which also a shift of increased vagal activity was found, in our case in response to increased blood pressure and ventricular contractility with dobutamine. Our results additionally show that with our setup a cardiac-modulated pattern can be recorded from the vagus nerve of the pig. Although details on morphology of the pig vagus nerve are unknown, the pig is considered superior to the rat as a model of the human cardiovascular system in both anatomy [15] and physiology [14]. Also, the pig and human vagus nerve diameter are larger than that of a rat, which may complicate recording.

Spectral analysis of the vagus nerve was also analyzed by Rozman and colleagues, who made recordings from the vagus nerve with an extraneural segmented cuff in dogs [30]. Power spectra were obtained that were found to provide organ-specific information on selected segments. These signals were not related to the cardiac cycle.

EMG from nearby vessels or neck musculature can disturb cervical vagus nerve recordings. Within a cuff the electrical activity of muscles can create a linear potential field. A tripolar cuff configuration can reduce EMG interference, because it takes a second order spatial derivative of a linear potential field, which will be zero in the ideal situation. An average reference configuration, or a multi-electrode configuration, may not suppress the EMG disturbance completely. However, the estimated SNR of the centre ring electrode, which corresponds to a tripolar derivation, was similar to the estimated SNR of the outer ring electrode. This shows that the tripolar derivation would not improve signal quality in our experiments. These results may indicate that EMG disturbance was low in our recordings and that other factors were more important contributors to the noise. It is also possible that EMG disturbances are recorded despite of the use of a tripolar derivation [31]. It should be noted that high pass filtering of the signal at a cut off frequency of $800 \mathrm{~Hz}$ improves SNR. Ensemble averaging will further reduce interference, assuming that it is not cardiac-modulated.

There were seven pigs in which a cardiac-modulated pattern was found on an outer ring electrode, but not on the centre electrode with the average reference configuration. This pattern is then not observed with a tripolar configuration, 
because the signal recorded with a tripolar derivation has the same pattern as the signal on the centre electrode using the average reference method. Poor electrode contact of the other electrodes or variations in fibre orientation may explain why only one out of three ring electrodes could record the cardiacmodulated signal. Average reference recording increases the odds of sensing a cardiac-modulated pattern, because it provides a signal from each electrode.

The cardiac-modulated patterns found in this chapter should be further examined in multiple ways, such as by direct manipulation of cardiac receptors. Additionally, cutting the afferent cardiac vagal innervation could be used to verify the origin of the observed modulation. If the modulation maintains after cutting, the signals are not of cardiac origin. Chronic recording without anaesthesia should be performed to analyze the signals in a more natural condition. Intrafascicular electrodes, such as the LIFE configuration, are possibly useful to improve selectivity and to reduce the need for ensemble averaging [32]. The difficulty of an intrafascicular electrode is to place it in the correct fascicle. Several attempts may be needed to find the optimal location.

These experiments were performed in anesthetized pigs, which may have affected the cardiovascular system. For example, isoflurane is known to decrease cardiac output [33] and induce vasodilation [34]. However, since anaesthesia was held constant during our experiments, we do not expect that anaesthetics have influenced our conclusions. Administration of morphine is not associated with significant cardiovascular side effects during isofluranemaintained anaesthesia in dogs [35]. Also, administration of dobutamine effectively influenced the cardiovascular system despite the anaesthesia, which was also observed in horses under isoflurane-maintained anaesthesia [36].

We were able to record cardiac-modulated signals from the vagus nerve using an extraneural cuff electrode. Changes in the cardiovascular system brought about by dobutamine could be observed in this extraneurally recorded pattern. The results suggest that changes in ventricular pressure, ventricular contractility and coronary pressure may be reflected in changes in the pattern of the vagal signal. Signals from receptors can provide relevant information that is not easily obtained in a non-invasive manner. For example, atrial mechanoreceptors with vagal afferents are known to show an increase in their activity during myocardial ischemia [9-10]. These and other pathological states like upcoming decompensation in heart failure or vulnerability for arrhythmias may possibly be observed in the extraneurally recorded cardiac-modulated vagal signal. This could provide a method for monitoring chronically, for example combined with an implanted vagus nerve stimulator as a closed-loop therapy for any of these diseases. 


\subsection{Conclusions}

Cardiac-modulated patterns can be recorded extraneurally from the vagus nerve. Vagal cardiac signals can more often be identified by using a multi-contact electrode cuff than a cuff with ring electrodes. Average reference recording increases the odds of sensing a cardiac-modulated pattern. During baseline condition, vagal activity can be increased compared to the average of the cardiac cycle in all phases of the cardiac cycle, but most often in the TP phase. When blood pressure and ventricular contractility increase with dobutamine, the cardiac-modulated signal shows highest vagal activity during the PQ and QRST phase. Thus, the cardiac vagal activity becomes more focused to systole when blood pressure, contractility, or both increase.

\section{References}

1. Arndt, J.O., P. Brambring, K. Hindorf, and M. Rohnelt (1971) Afferent impulse traffic from atrial a-type receptors in cats - does a-type receptor signal heart rate Pflug Arch Eur J Phy, 326(4), p. 300-15.

2. $\quad$ Arndt, J.O., P. Brambring, K. Hindorf, and M. Rohnelt (1974) Afferent discharge pattern of atrial mechanoreceptors in cat during sinusoidal stretch of atrial strips insitu J Physiol-London, 240(1), p. 33-52.

3. Brown, A.M. (1965) Mechanoreceptors in or near the coronary arteries J Physiol, 177, p. 203-14.

4. Geiger, S.R., Handbook of physiology: A critical, comprehensive presentation of physiological knowledge and concepts. Section 2: The cardiovascular system. Vol. 3. 1983, Washington: American Physiological Society.

5. Paintal, A.S. (1955) A study of ventricular pressure receptors and their role in the bezold reflex Q J Exp Physiol Cogn Med Sci, 40(4), p. 34863.

6. Thoren, P.N. (1976) Atrial receptors with nonmedullated vagal afferents in the cat. Discharge frequency and pattern in relation to atrial pressure Circ Res, 38(5), p. 357-62.

7. $\quad$ Morris, G.L., 3rd and W.M. Mueller (1999) Long-term treatment with vagus nerve stimulation in patients with refractory epilepsy. The vagus nerve stimulation study group e01-e05 Neurology, 53(8), p. 1731-5.

8. Schwartz, P.J. and G.M. De Ferrari (2009) Vagal stimulation for heart failure: Background and first in-man study Heart Rhythm, 6(11), p. S76-S81.

9. Cerati, D. and P.J. Schwartz (1991) Single cardiac vagal fibre activity, acute myocardial-ischemia, and risk for sudden-death Circ Res, 69(5), p. $1389-401$.

10. Recordati, G., P.J. Schwartz, M. Pagani, A. Malliani, and A.M. Brown (1971) Activation of cardiac vagal receptors during myocardial ischemia Experientia, 27(12), p. 1423-4. 
11. Agostoni, E., J.E. Chinnock, M.D.B. Daly, and J.G. Murray (1957) Functional and histological studies of the vagus nerve and its branches to the heart, lungs and abdominal viscera in the cat $\mathrm{J}$ Physiol-London, 135(1), p. 182-205.

12. Botar, J., G. Popjak, and A. Bense, Die fasern des nervus vagoaccessorius beim menschen und bei saugetieren. 1937, Leipzig: Johan Ambrosius Barth.

13. Robinson, A.J. and L. Snyder-Mackler, Clinical electrophysiology : Electrotherapy and electrophysiologic testing. 3rd ed. 2008, Philadelphia: Wolters Kluwer/Lippincott Williams \& Wilkins. xv, p. 555

14. Arora, R.C., M. Waldmann, D.A. Hopkins, and J.A. Armour (2003) Porcine intrinsic cardiac ganglia Anat Rec Part a, 271A(1), p. 249-58.

15. Crick, S.J., M.N. Sheppard, S.Y. Ho, L. Gebstein, and R.H. Anderson (1998) Anatomy of the pig heart: Comparisons with normal human cardiac structure J Anat, 193, p. 105-19.

16. Sahni, D., G.D. Kaur, Harjeet, and I. Jit (2008) Anatomy \& distribution of coronary arteries in pig in comparison with man Indian J Med Res, 127(6), p. 564-70.

17. Swindle, M.M. and A.C. Smith (1998) Comparative anatomy and physiology of the pig Scand J Lab Anim Sci, 25, p. 11-21.

18. Tyler, D.J. and D.D.M. Durand (2002) Functionally selective peripheral nerve stimulation with a flat interface nerve electrode IEEE T Neur Sys Reh, 10(4), p. 294-303.

19. Ludwig, K.A., R.M. Miriani, N.B. Langhals, M.D. Joseph, D.J. Anderson, and D.R. Kipke (2009) Using a common average reference to improve cortical neuron recordings from microelectrode arrays $\mathrm{J}$ Neurophysiol, 101(3), p. 1679-89.

20. Offner, F.F. (1950) The EEG as potential mapping - the value of the average monopolar reference Electroen Clin Neuro, 2(2), p. 213-4.

21. Randall, D.C. (1974) Concurrent measurement of left-ventricular dpdtmax, isometric contractile-force and cardiac loading in intact monkey Cardiology, 59(5), p. 304-18.

22. Rahal, M., J. Taylor, and N. Donaldson (2000) The effect of nerve cuff geometry on interference reduction: A study by computer modeling IEEE Trans Biomed Eng, 47(1), p. 136-8.

23. Stein, R.B., D. Charles, L. Davis, J. Jhamandas, A. Mannard, and T.R. Nichols (1975) Principles underlying new methods for chronic neural recording Can J Neurol Sci, 2(3), p. 235-44.

24. Nikolic, Z.M., D.B. Popovic, R.B. Stein, and Z. Kenwell (1994) Instrumentation for eng and emg recordings in fes systems IEEE Trans Biomed Eng, 41(7), p. 703-6. 
25. Rahal, M., J. Winter, J. Taylor, and N. Donaldson (2000) An improved configuration for the reduction of emg in electrode cuff recordings: A theoretical approach IEEE Trans Biomed Eng, 47(9), p. 1281-4.

26. Harreby, K.R., C. Sevcencu, and J.J. Struijk (2011) Ictal and peri-ictal changes in cervical vagus nerve activity associated with cardiac effects Med Biol Eng Comput.

27. Paintal, A.S. (1953) The conduction velocities of respiratory and cardiovascular afferent fibres in the vagus nerve J Physiol-London, 121(2), p. 341-59.

28. Lichtwarck-Aschoff, M., R. Beale, and U.J. Pfeiffer (1996) Central venous pressure, pulmonary artery occlusion pressure, intrathoracic blood volume, and right ventricular end-diastolic volume as indicators of cardiac preload J Crit Care, 11(4), p. 180-8.

29. Fox, I.J., D.A. Gerasch, and J.J. Leonard (1977) Left ventricular mechanoreceptors: A haemodynamic study J Physiol, 273(2), p. 405-25.

30. Rozman, J. and S. Ribaric (2007) Selective recording of electroneurograms from the left vagus nerve of a dog during stimulation of cardiovascular or respiratory systems Chinese J Physiol, 50(5), p. 240-50.

31. Andersen, M.P., M. Munch, W. Jensen, P. Sørensen, and C.F. Eder, Chronic cuff electrode recordings from walking göttingen mini-pigs, in 33rd Annual International Conference of the IEEE EMBS. 2011: Boston, Massachusetts USA.

32. Zheng, X., J. Zhang, T. Chen, and Z. Chen (2008) Recording and stimulating properties of chronically implanted longitudinal intrafascicular electrodes in peripheral fascicles in an animal model Microsurgery, 28(3), p. 203-9.

33. Steffey, E.P., and Howland, D. Jr (1980) Comparison of circulatory and respiratory effects of isoflurane and halothane anesthesia in horses. Am J Vet Res, 41, 821-825

34. Schwinn, D.A., R. W. McIntyre, and J.G. Reves (1990) IsofluraneInduced Vasodilation: Role of the $\alpha$-Adrenergic Nervous System Anesth Analg 71(5) p.451-9

35. Keegan, R.D., Greene, S.A., and Weil, A.B. Cardiovascular effects of epidurally administered morphine and a xylazine-morphine combination in isoflurane-anesthetized dogs, Am J Vet Res 56(4) p. 496-500

36. A. De Vries, J.C. Brearley, and P.M. Taylor (2009) Effects of dobutamine on cardiac index and arterial blood pressure in isofluraneanaesthetized horses under clinical conditions, J Vet Pharmacol Therap 32(4), p. 353-8 


\section{Chapter 3}

\section{An indirect component in the evoked compound action potential of the vagus nerve}

Simone Ordelman

Lilian Kornet

Richard Cornelussen

Rik Buschman

Peter Veltink

J Neural Eng, 7(6):066001, 2010 


\section{Abstract}

The vagus nerve plays a vital role in the regulation of the cardiovascular system. It not only regulates the heart but also sends sensory information from the heart back to the brain. We hypothesize that the evoked vagus nerve compound action potential contains components that are indirect via the brain stem or coming via the neural network on the heart. In an experimental study in fifteen pigs, we identified four components in the evoked compound action potentials. The fourth component was found to be an indirect component, which came from the periphery. The latency of the indirect component increased when heart rate and contractility were decreased by burst stimulation $(\mathrm{P}=0.01 ; \mathrm{n}=7)$. When heart rate and contractility were increased by dobutamine administration, the latency of the indirect component decreased $(\mathrm{P}=0.01 ; \mathrm{n}=9)$. This showed that the latency of the indirect component of the evoked compound action potentials may relate to the state of the cardiovascular system. 


\subsection{Introduction}

When electrically stimulating a nerve, action potentials can be evoked in the nerve fibres. The cumulative response of all action potentials is called the compound action potential (CAP). Fibre types in peripheral nerves are classified into A, B, and C types [1]. Type A has the lowest threshold for evoking an action potential, followed by type B and type C. A fibres are large $(1-20 \mu \mathrm{m}$ in diameter), myelinated, and have high conduction velocities $(6-120 \mathrm{~m} / \mathrm{s})$. B fibres are smaller (less than $3 \mu \mathrm{m}$ in diameter), myelinated, and have lower conduction velocities $(2-18 \mathrm{~m} / \mathrm{s})$. C fibres are the smallest type (less than $1 \mu \mathrm{m}$ in diameter), unmyelinated, and have the lowest conduction velocities (less than $2 \mathrm{~m} / \mathrm{s})$. Type A fibres are subdivided into A $\alpha(12-20 \mu \mathrm{m}), \mathrm{A} \beta(5-12 \mu \mathrm{m}), \mathrm{A} \gamma(2-8$ $\mu \mathrm{m})$ and $\mathrm{A} \delta(1-5 \mu \mathrm{m})$.

The cervical vagus nerve comprises fibres with a diameter ranging from $1 \mu \mathrm{m}$ to $20 \mu \mathrm{m}$ [2]. Only about $16 \%$ of the total fibre content is myelinated [2]. The largest myelinated fibres, above $12 \mu \mathrm{m}$ in diameter, innervate the laryngeal muscles [2]. Almost all medium sized myelinated fibres, 6 to $12 \mu \mathrm{m}$ in diameter, go to oesophageal, bronchial and cardiac branches of the vagus [2].

The vagus nerve plays a vital role in autonomic control of the heart. Together with the sympathetic system, the vagus nerve regulates heart rate, blood pressure, contractility and AV-node conductivity. A multiplicity of interactions occurs between neurons of the sympathetic and parasympathetic system [3]. Cardiovascular reflexes are an important part of maintaining a stable cardiovascular state [4]. Changes in cardiovascular parameters such as blood pressure and oxygenation lead to a change in afferent firing pattern of cardiac receptors. In response, brain centres induce appropriate efferent vagal and sympathetic signalling which leads to an adequate cardiac response. The vagus nerve is part of both baroreceptor and chemoreceptor reflexes [5].

The final relay station of cardiac neurons is the intrinsic cardiac network, which consists of autonomic ganglia and axons located on the heart itself or along the large vessels within the pericardium. This network comprises parasympathetic neurons, sympathetic neurons and interconnecting neurons. It transduces neural inputs and coordinates cardiac electrical and mechanical parameters. Detailed information concerning the signalling pathways and coordination within the cardiac neuronal hierarchy is scarce [3].

Even though the vagus nerve is part of this complex network with many reflexes, never has an indirect component been found in the vagal CAP. An explanation may be that in commonly used experimental setup the distinction between a direct and an indirect component can not be made, since the direction and velocity along the nerve of the components are not assessed directly. In these studies, it is assumed that all components originate at the site of stimulation and travel a direct path from stimulation site to recording site. 
We hypothesized that the vagal CAP contains not only the response of the fibres directly activated by electrical stimulation, but also responses that occur as part of the neurocardiac control system. Possible indirect components that are part of a reflexive pathway via the brainstem or via the neural network on the heart may be found. We expected to find both an indirect component via the brainstem that is part of the baroreceptor reflex pathway and an indirect component coming from the neural network on the heart. With changes in the cardiovascular system, we expected to see changes in amplitude and latency, caused by changes in firing rate of baroreceptors or in relay time at the neural network respectively. Such components could be clinically relevant for monitoring the heart. Cardiovascular diseased states may be monitored via the vagus nerve [6].

In this chapter the question is addressed whether the evoked CAP of the cervical vagus nerve includes an indirect component coming from the periphery or an indirect component coming from the brain stem. Furthermore, it is assessed whether this indirect component depends on the state of the cardiovascular system.

\subsection{Methods}

\subsubsection{Instrumentation and experimental setup}

Vagus nerve electrical stimulation and sensing experiments were performed in fifteen female Dutch Landrace pigs. The pig was premedicated with $0.5 \mathrm{mg} / \mathrm{kg}$ Morphine 20 minutes before induction with Zoletil (Telazol, $3 \mathrm{mg} / \mathrm{kg}$ ) and xylazine $(0.5 \mathrm{mg} / \mathrm{kg})$. The pig was anesthetized with a combination of isoflurane $(1-1.5 \%)$ and fentanyl $(6-10 \mu \mathrm{g} / \mathrm{kg} / \mathrm{hr})$. At the end of the experimental day, the pig was euthanized with an overdose of pentobarbital.

In all fifteen pigs, the left cervical vagus nerve was carefully dissected free from the surrounding tissue. The nerve was freed for a length of approximately ten centimetres, limited by anatomical constraints.

Three custom-made self-coiling cuff electrodes were placed. Each cuff was 15 $\mathrm{mm}$ long and had three circular Pt/Ir electrode contacts with an interelectrode distance of $4 \mathrm{~mm}$ (figure $3.1 \mathrm{~b}$ ). The cuffs were made in various diameters (2, 3.5 and $5 \mathrm{~mm}$ ), to ensure a proper fit around the nerve. Two electrodes were placed as caudal as possible; the third electrode was placed as rostral as possible (figure 3.1a). The distance between the electrodes was measured and a picture of the cuffs around the nerve was taken. The most caudal electrode was used for stimulation, the other two for recording.

The nerve was positioned back within the surrounding tissue. The wound was kept open, but it was ensured that the cuffs were surrounded by tissue and that there was enough fluid for a proper electrical contact and drying of the nerve was avoided. If signals diminished during the experiment, saline solution was added to the wound. 
In four out of fifteen pigs, the right cervical vagus nerve was also dissected free on which one cuff was placed (figure 3.1a). This cuff on the right vagus nerve was used for recording. With this cuff, it could be observed if indirect components travelled along the right vagus nerve as a result of stimulation of the left vagus nerve.
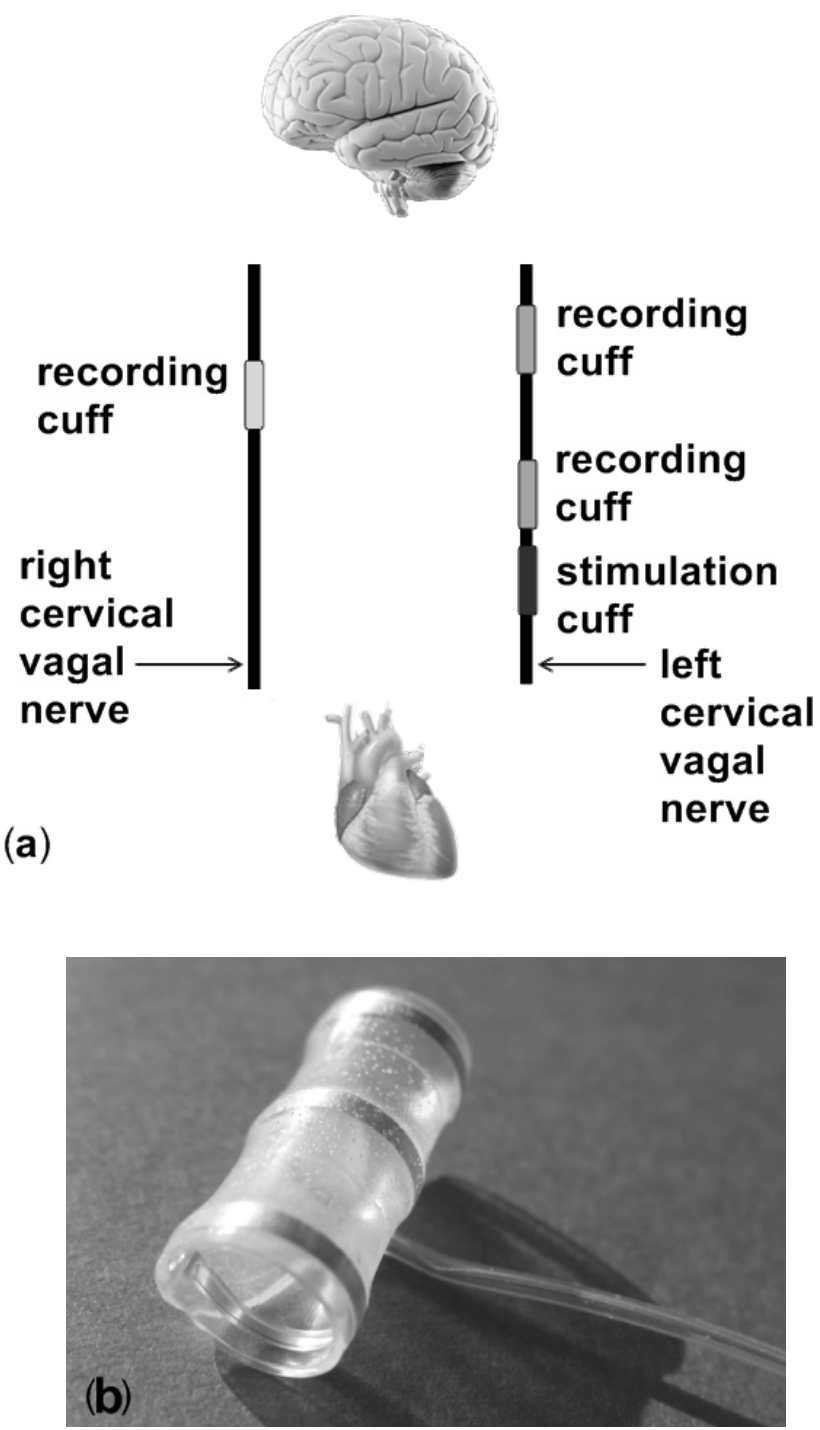

Figure 3.1: Schematic representation of electrode placement (a) and the electrode cuff (b). In all fifteen pigs, three cuffs were placed on the left vagus nerve. In four out of fifteen pigs, an additional recording cuff was placed on the right vagus nerve. 
Electrical stimulation was performed with a custom-made stimulator. ENG signals were sampled at $20 \mathrm{kHz}$ and amplified by a TMSi Refa amplifier (TMS international, Oldenzaal, The Netherlands). ECG was recorded with contact electrodes placed on shaved paws. Left ventricular blood pressure was recorded with a Millar catheter. ECG and blood pressure recordings were sampled at $2048 \mathrm{~Hz}$ and amplified with a TMSi Porti amplifier (TMS international, Oldenzaal, The Netherlands). All data were stored on a laptop using PortiLab2 software (TMS international, Oldenzaal, The Netherlands).

Signals were measured using the average reference method [7], which uses the average of all signals as a reference. We used this method because it eliminates the need for bipolar or tripolar recording and allows recordings of ENG signals from all three electrode sites on a cuff separately.

\subsubsection{Experimental protocol}

Initially, the left vagus nerve was stimulated at a pulse width of $300 \mu$ s and a stimulation frequency of $1 \mathrm{~Hz}$. Stimulation amplitude was set at $0.05 \mathrm{~mA}$, $0.1 \mathrm{~mA}$, and increasing from $0.2 \mathrm{~mA}$ to $5 \mathrm{~mA}$ in increments of $0.2 \mathrm{~mA}$. At each amplitude ten pulses were delivered to the nerve. This phase of the protocol was used to determine the stimulation threshold at which each component of the CAP occurred first.

Subsequently, burst stimulation was performed on the left vagus nerve. Pulse width was kept at $300 \mu \mathrm{s}$. Stimulation frequency was set to $10 \mathrm{~Hz}, 20 \mathrm{~Hz}, 30$ $\mathrm{Hz}, 40 \mathrm{~Hz}$, or $50 \mathrm{~Hz}$. Amplitude was varied between $1 \mathrm{~mA}$ and $5 \mathrm{~mA}$ for each frequency, until the setting with the largest effect on heart rate was found. Stimulation was maintained for 60 seconds, or until unwanted side effects occurred (such as complete heart block or digestive reflex).

Burst stimulation was performed to create an additional cardiac state of reduced heart rate, induced by burst stimulation, so that CAPs between this additional state and baseline condition could be compared. Data were excluded from the analysis if the effect of burst stimulation on heart rate was less than ten percent.

Dobutamine $(5 \mathrm{~mL} / \mathrm{h})$ was administered after burst stimulation was completed. Dobutamine (a sympathomimetic drug) increases heart rate and left-ventricular contractility by stimulating the $\beta_{1}$ receptors on the heart. Stimulation of the left vagus nerve at $1 \mathrm{~Hz}$ was repeated to inspect the CAPs in the additional cardiac state, induced by dobutamine. These CAPs could then be compared to baseline condition. Data were excluded from the analysis if the effect of dobutamine on heart rate was less than ten percent or if CAPs could not be recorded.

At the end of the experiment an extra test was performed to verify the direction of the components in the CAP. In three pigs, the left vagus nerve was cut rostral to the electrodes. Stimulation at $1 \mathrm{~Hz}, 300 \mu$ s and $5 \mathrm{~mA}$ was repeated. If components of the CAPs had disappeared at this point, these component 
originated in the brain stem. In two pigs the nerve was subsequently cut caudal to the electrodes and stimulation was repeated. If components had disappeared at this point, these components came from the periphery.

\subsubsection{Signal analysis}

Offline signal analysis was performed in Matlab R2007a (The Mathworks Inc.). ENG and ECG were detrended to remove drift. R-tops of the ECG were detected automatically and confirmed by visual inspection. Left ventricular contractility was assessed using maximum values of the gradient of left ventricular pressure: $\left(\frac{d P_{L V}}{d t}\right)_{\text {peak }}$ [8]. This parameter will be called contractility.

The mean signal of each electrode cuff was subtracted from the individual electrode sites on that cuff. In this way common signals were removed. Stimulus artefacts in the ENG were automatically detected and used to overlap CAPs from a train of stimuli to create an ensemble average. For $1 \mathrm{~Hz}$ stimulation, all ten pulses delivered at the same amplitude were included in the ensemble. For burst stimulation at $10 \mathrm{~Hz}$ to $50 \mathrm{~Hz}$, the final 100 pulses of a burst delivered at the same amplitude were used for the ensemble, since at this point heart rate had changed most.

The stimulus artefact was then removed from the plot by subtracting a template. This template was obtained by stimulating at a stimulation level of $1 \mathrm{~Hz}, 300 \mu \mathrm{s}$ and $5 \mathrm{~mA}$ and recording the stimulus artefact in the tissue without contact with the nerve. When applying the template, the amplitude of the stimulus artefact was scaled to match the actual stimulus artefact.

The electrode furthest away from the stimulation site was used to analyze the CAPs, since at this point - due to the difference in conduction speed of the various components - the least overlap of components was expected. If this electrode did not provide a signal, a closer electrode was used.

For each component of the CAP, the stimulation amplitude at which it first occurred was noted as the stimulation threshold. The threshold was used to identify the components: a low stimulation threshold corresponds with A fibres, a high stimulation threshold with $\mathrm{B}$ or $\mathrm{C}$ fibres. The CAP that resulted from stimulation at the maximum amplitude of $5 \mathrm{~mA}$ - in which all components were visible - was used to determine velocity and direction of each component. The velocity along the nerve of each component was used to determine the exact fibre type (e.g. A $\alpha$ or $A \beta$ ), in accordance with conduction velocities reported in the literature [1].

To determine velocity, the interelectrode distance was divided by the difference in latency between consecutive electrode sites. The velocity was estimated both by using two recordings on the same cuff and by using two recordings on separate cuffs, if all signals could be recorded. In this way the error that occurred in the estimation was minimized. 
Latency of a component was defined as the time between the peak of the stimulus artefact and the highest initial slope of the component (figure 3.2). The slope was used since it is less susceptible than peak detection to small changes due to noise. Initial slopes of individual components were identified by their initial occurrence at different stimulation levels. The order in which a component occurred on the consecutive recording sites was analyzed to determine the direction. Amplitude of a component was defined as peak-to-peak value of a component (figure 3.3).

A distinction was made between direct and indirect components. Direct components have a velocity along the nerve that matches the time it took to reach the recording electrode and they come from the direction of the stimulation electrode. If the direction of a component indicates that it does not come from the stimulation electrode, it is an indirect component that originated in the brain stem. If the component comes from the direction of the stimulation electrode, but has a velocity along the nerve that is too high for the time it took to reach the recording cuff (long latency), it has travelled a longer path and is therefore an indirect component that comes from the periphery.

The CAPs were compared between three conditions: baseline, decreased heart rate and contractility caused by burst stimulation, and increased heart rate and contractility caused by dobutamine. Latency and amplitude of each component were compared between these conditions.

All collected data was tested for normality with a Shapiro-Wilk test. Parametric data is represented as mean $\pm \mathrm{SD}$, other data as median. A paired samples t-test was performed to test the differences statistically with parametric data. With other data a Wilcoxon Signed Rank test was used. Statistical significance was tested at the 0.05 level. 


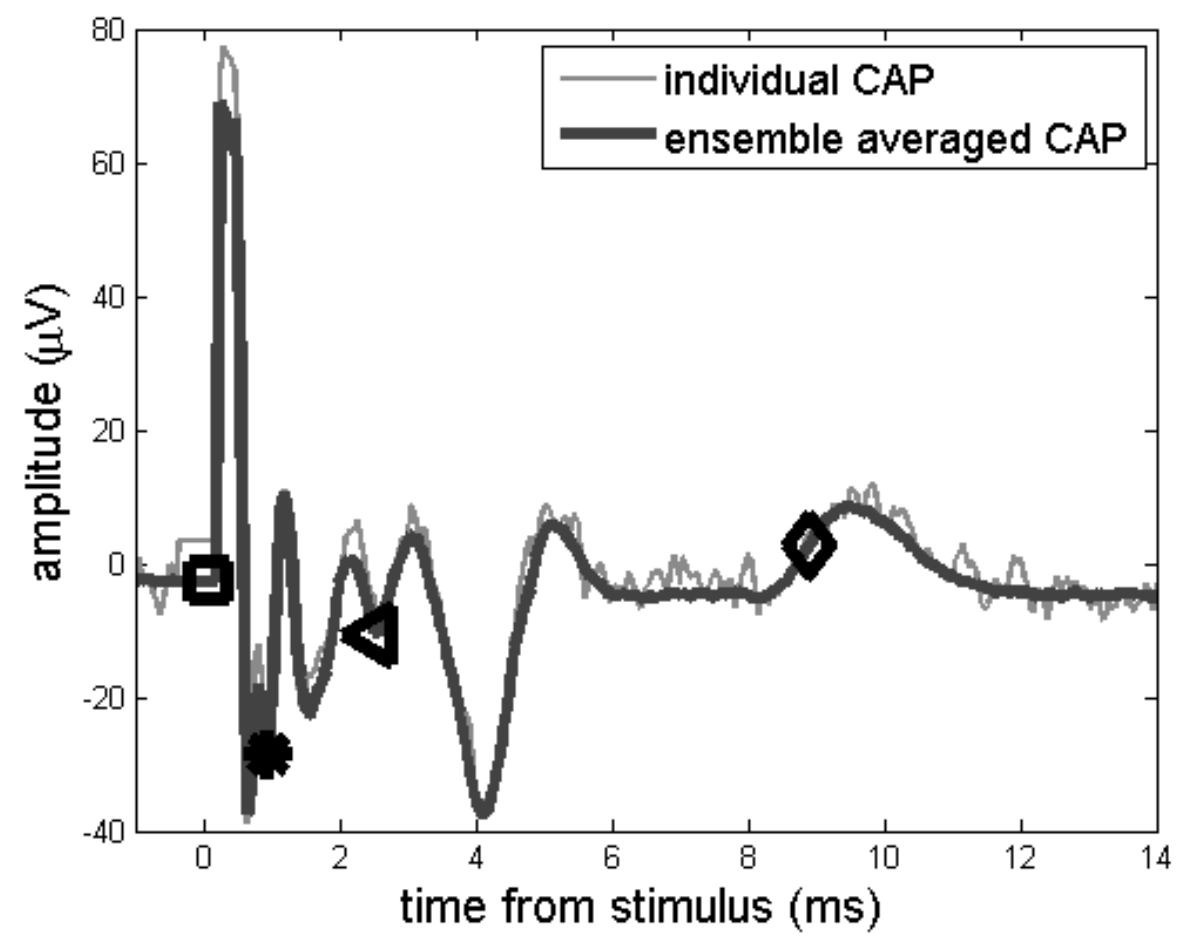

Figure 3.2: Individual recording of a CAP and the ensemble averaged signal of 100 CAPs. Noise levels for individual recordings were low enough to identify the components from the individual CAPs. Ensemble averaging further reduced the noise level. The stimulus artefact (at $t=0)$ has been removed from the plot with a template. Markers indicate latency determined based on the highest initial slope of the four components: $A \alpha(\square), A \beta(*), B(\Delta)$ and $A_{\text {ind }}(\nabla)$. The components were distinguished based on stimulus threshold. Example from pig 3. 


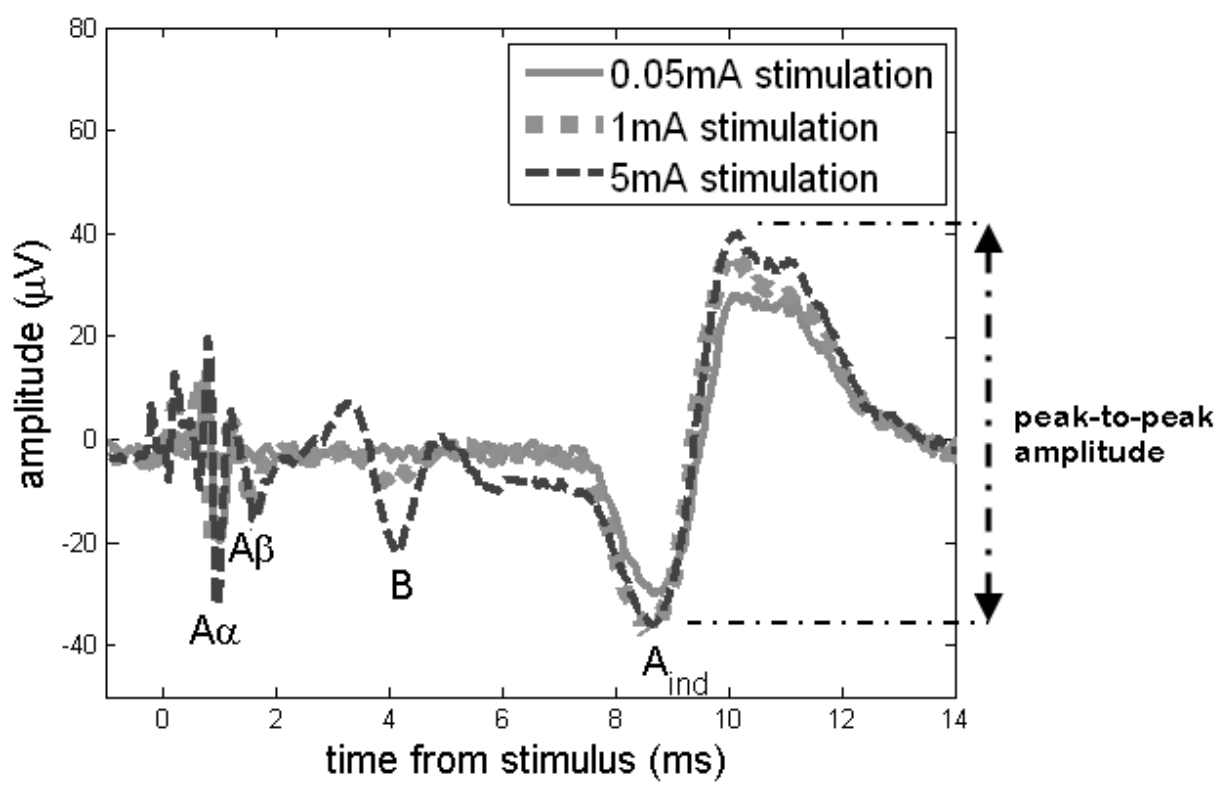

Figure 3.3: CAP at varying stimulation levels: $0.05 \mathrm{~mA}(-) ; 1 \mathrm{~mA}(. .$.$) ; and 5 \mathrm{~mA}(---)$. The stimulus is applied at $t=0$. The stimulus artefact is reduced by subtracting $a$ template. The components $A \alpha, A \beta, B$ and $A_{\text {ind }}$ are indicated in the plot. Averages of 10 CAPs are shown at each stimulation amplitude. The arrow indicates peak-to-peak amplitude of the $A_{\text {ind }}$ component. Example from pig 4.

\subsection{Results}

\subsubsection{The indirect component}

In thirteen out of fifteen pigs, the recorded CAP consisted of four components. An example is shown in figure 3.3. In the other two pigs, the stimulus artefact obscured the first three components, but the fourth component could be identified. The distance between the stimulation site and the recording electrode used for analyzing the CAPs was 4.1 to $6.0 \mathrm{~cm}$.

The first two components had a low threshold $(0.13 \pm 0.08 \mathrm{~mA}$ for the first and $0.49 \pm 0.40 \mathrm{~mA}$ for the second). These components were identified as $\mathrm{A} \alpha$ and $\mathrm{A} \beta$ respectively. The latency of the A $\alpha$ component was $0.50 \pm 0.34 \mathrm{~ms}$ and it had a velocity of $73 \pm 16 \mathrm{~m} / \mathrm{s}$. Analysis of the direction by looking at recordings on consecutive electrodes showed it came from the direction of the stimulation cuff. 
Latency of the $A \beta$ component was $1.08 \pm 0.43 \mathrm{~ms}$ and velocity was $67 \pm 20$ $\mathrm{m} / \mathrm{s}$. It also came from the direction of the stimulation cuff.

The third component had the highest threshold $(2.17 \pm 1.27 \mathrm{~mA})$ and was identified as a component of B fibres. It had the lowest velocity $(20 \pm 9 \mathrm{~m} / \mathrm{s})$, and a latency of $3.13 \pm 1.41 \mathrm{~ms}$. It came from the direction of the stimulation cuff.

The fourth component had a threshold higher than that of the A $\alpha$ component and lower than that of the $\mathrm{A} \beta$ component $(0.16 \pm 0.13 \mathrm{~mA})$, but it had the longest latency of all components $(8.13 \pm 1.12 \mathrm{~ms})$. Velocity along the nerve was estimated at $69 \pm 17 \mathrm{~m} / \mathrm{s}$. Based on velocity, it was identified as coming from A fibres. It came from the direction of the stimulation cuff.

The $\mathrm{A} \alpha, \mathrm{A} \beta$ and $\mathrm{B}$ component have a threshold, velocity and direction that combined show that these are direct components. The fourth component, however, comes from the direction of the stimulation electrode, but has a high velocity that does not match its long latency and therefore it is an indirect component. Since it is indirect and its velocity indicates that it comes from A fibres, this component will be called $\mathrm{A}_{\text {ind }}$. A difference in latency and shape between recordings from consecutive electrode cuffs, also supports that it is not a far field potential (figure 3.4).

An overview of the amplitudes of all components as a function of stimulation amplitude for all pigs is shown in figure 3.5.

In the four pigs in which an additional electrode cuff was placed on the right vagus nerve, the $A_{\text {ind }}$ component was visible on both the left and right vagus nerve when the left vagus nerve was stimulated (figure 3.6). The latency of the $A_{\text {ind }}$ component on the right vagus nerve was $8.7 \pm 0.6 \mathrm{~ms}$, which is a difference of $-0.1 \pm 1 \mathrm{~ms}$ with the latency on the left vagus nerve. The shape of the $\mathrm{A}_{\text {ind }}$ component was not identical on both nerves (figure 3.6). The $A \alpha, A \beta$ and $B$ components of the CAP did not occur on the right vagus nerve when the left vagus nerve was stimulated.

In three pigs, the left vagus nerve was cut rostral to the electrodes at the end of the experiment. All components were still present when electrical stimulation was repeated. In two pigs the vagus nerve was subsequently cut caudal to the electrodes. When electrical stimulation was repeated, the $\mathrm{A}_{\text {ind }}$ component had disappeared, but the other components were still present. 


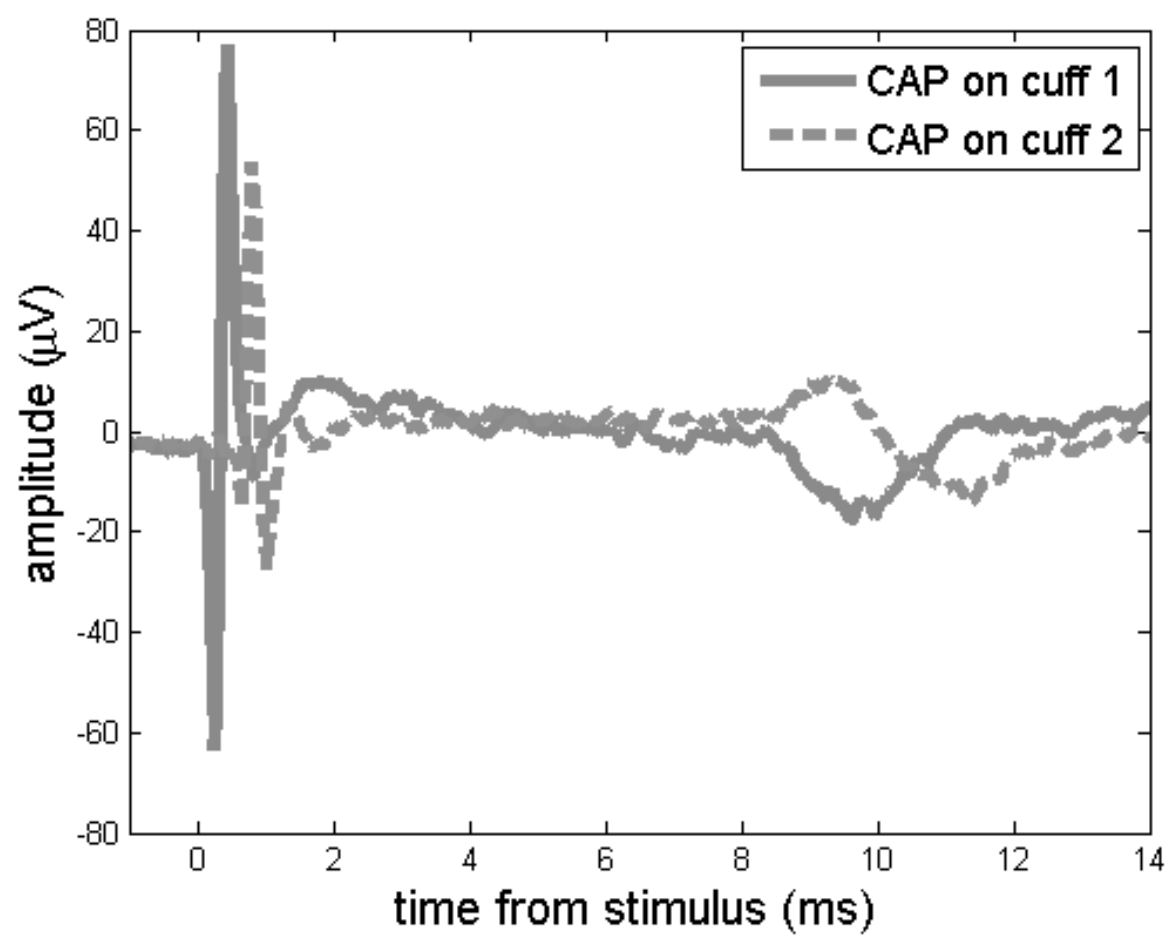

Figure 3.4: Example of the CAP on the two cuffs on the left vagus nerve at a stimulation

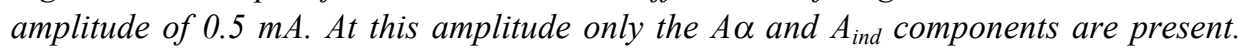
The stimulus artefact at $t=0$ is removed from the plot by subtracting a template. The delay between two consecutive electrode cuffs is approximately $1 \mathrm{~ms}$ in this example for the $A_{\text {ind }}$ component and about $0.5 \mathrm{~ms}$ for the A $\alpha$ component. The distance between the two recording sites was about $4.3 \mathrm{~cm}$. Both the delay between electrode cuffs and the difference in shape in the $A_{\text {ind }}$ component show that it is not a far field potential. Example from pig 2. 


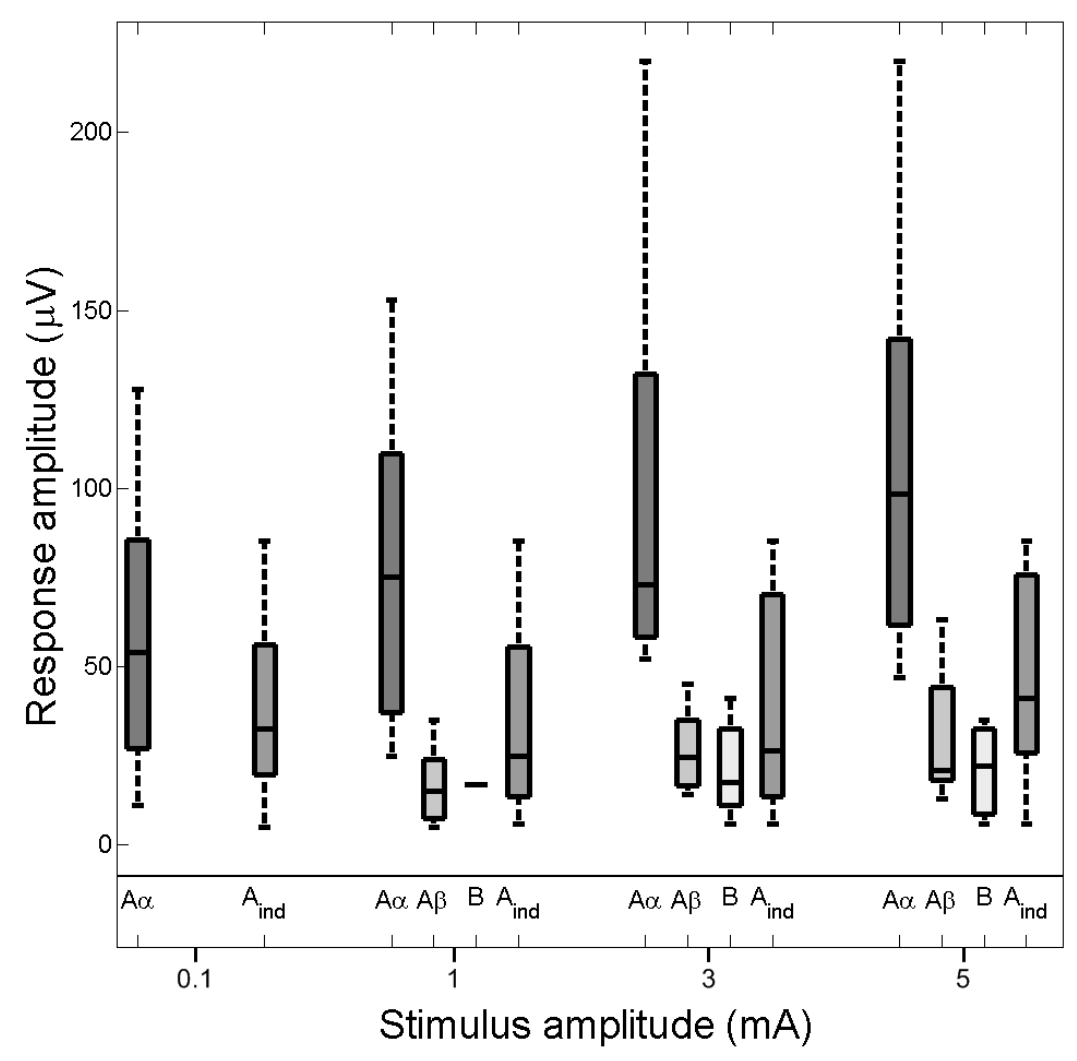

Figure 3.5: Components of the CAP at increasing stimulation amplitudes in all pigs $(n=15)$. Each bar represents the peak-to-peak amplitude of a component at a certain stimulation amplitude. Components $A \beta$ and $B$ did not occur at a stimulation amplitude of $0.1 \mathrm{~mA}$ in any of the pigs. Component $B$ occurred at a stimulation amplitude of $1 \mathrm{~mA}$ in only one pig. 


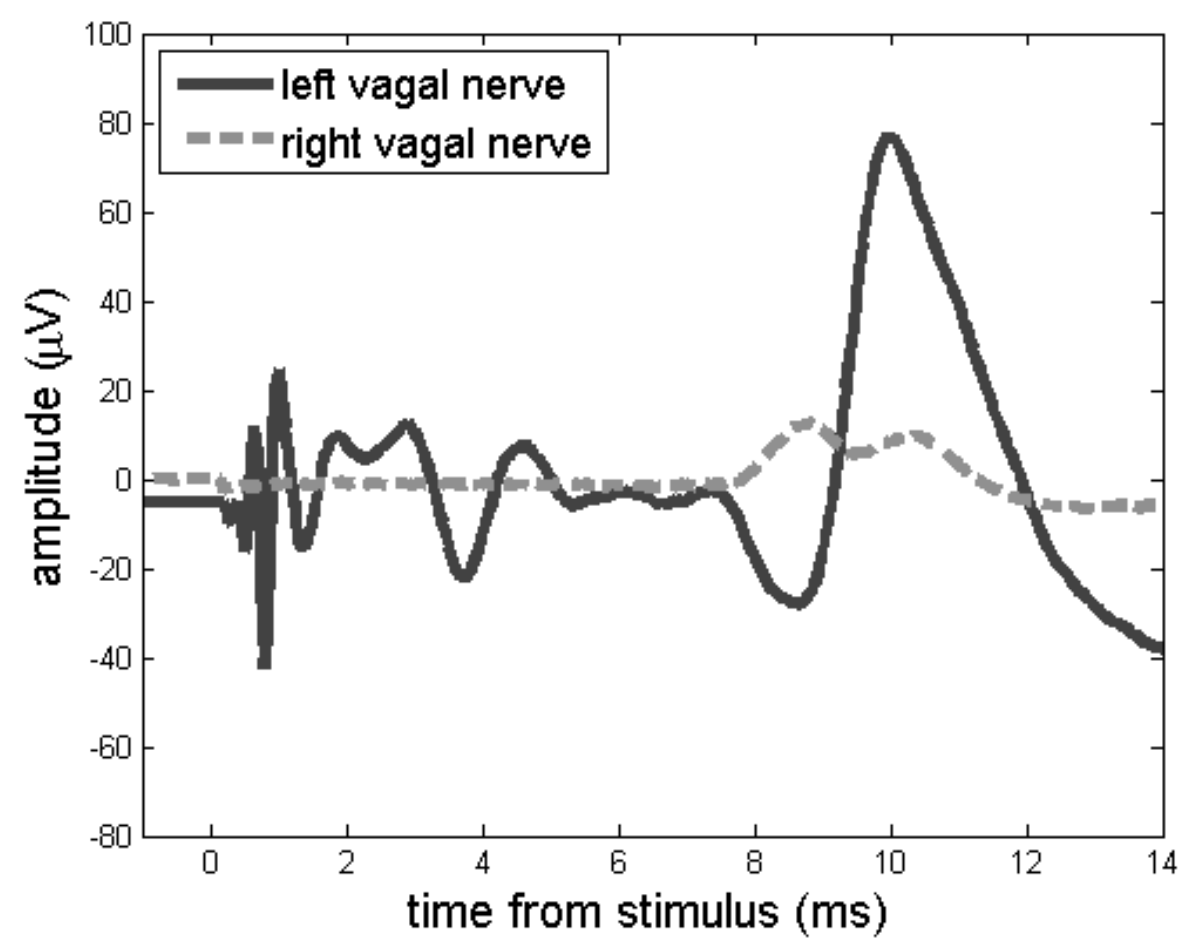

Figure 3.6: Ensemble average plot of a CAP in the left (-) and right (--) vagus nerve when stimulating the left vagus nerve at $300 \mu \mathrm{s}$ and $5 \mathrm{~mA}$. Only the fourth, indirect component is visible in the right vagus nerve. Its shape differs from the left vagus nerve, possibly due to a difference in dispersion. The latency of this component in the right vagus nerve is $0.2 \mathrm{~ms}$ larger than in the left vagus nerve in this example. The stimulus artefact (at $t=0)$ has been removed from the plot by subtracting a template. Example from pig 4.

\subsubsection{The indirect component and burst stimulation}

Measurements in which burst stimulation decreased heart rate by more than ten percent were included in the analysis. Out of the fifteen pigs, seven pigs could be included in this selection. For all these seven pigs, the stimulation parameters that resulted in the highest decrease of heart rate were $50 \mathrm{~Hz}$ and $5 \mathrm{~mA}$.

For each of the seven pigs, the CAPs during decreased heart rate caused by burst stimulation were compared to the CAPs during baseline, i.e. at $1 \mathrm{~Hz}$ stimulation. Heart rate during burst stimulation was significantly decreased compared to the baseline recordings (by $43 \pm 33 \%$; $=0.01 ; n=7$ ). In six out of seven pigs contractility could be recorded, which showed a median decrease of $12 \%$ with burst stimulation, which was statistically significant $(\mathrm{P}=0.03 ; \mathrm{n}=6)$. 
During burst stimulation a longer latency (by $0.73 \pm 0.5 \mathrm{~ms}$ ) of the $\mathrm{A}_{\text {ind }}$ component was found in all seven pigs (see figure 3.7 for a typical example). This effect was found statistically significant $(\mathrm{P}=0.01 ; \mathrm{n}=7)$ with the paired $\mathrm{t}$ test.

The B component showed an increase in latency (of $0.28 \pm 0.11 \mathrm{~ms}$ ) in four out of the seven pigs. In one pig latency of the B component decreased by $0.5 \mathrm{~ms}$. In the other two pigs, the $\mathrm{B}$ component could not be distinguished. The effects on latency of the $\mathrm{B}$ component were not statistically significant $(\mathrm{P}=0.445 ; \mathrm{n}=5)$. Latency of the $A \alpha$ and $A \beta$ components of the CAP did not change with burst stimulation. No significant change was observed in the amplitude of any component.

When $1 \mathrm{~Hz}$ stimulation was repeated after burst stimulation, the latency of all components returned to baseline values in all seven pigs.

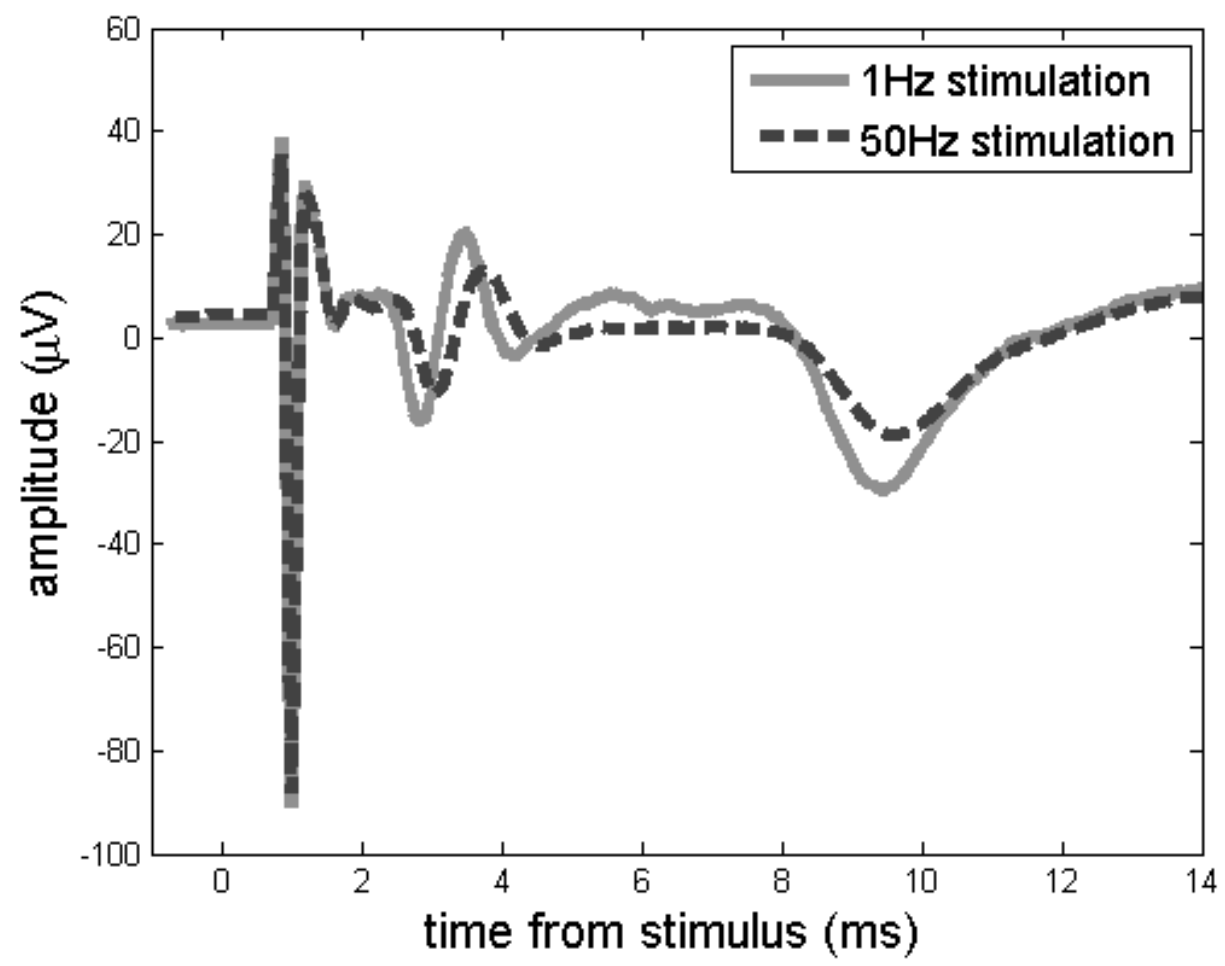

Figure 3.7: Example of the effect of burst stimulation on the CAP. Stimulation in this example decreased heart rate substantially. An increase in latency of the $A_{\text {ind }}$ component (which occurs around $t=8 m s$ in this example) was found for all pigs $(n=7)$. The stimulus artefact (at $t=0)$ has been removed from the plot by subtracting a template. Example from pig 5. 


\subsection{The indirect component and dobutamine}

When dobutamine was added, in nine out of fifteen pigs heart rate increased by more than ten percent and CAPs could be recorded. These nine pigs were included in the analysis. Heart rate increased substantially $(60 \pm 36 \% ; \mathrm{P}=0.001$; $\mathrm{n}=9$ ). The increase in heart rate occurred gradually and a period of up to one hour was needed for stabilization. Contractility showed a median increase of $72 \%(\mathrm{P}=0.01 ; \mathrm{n}=9)$.

For each of the nine pigs, the CAPs during increased heart rate caused by dobutamine were compared to the CAPs during baseline, i.e. without dobutamine. In all these nine pigs, latency of the $\mathrm{A}_{\text {ind }}$ component decreased (figure 3.8). The latency showed a median decrease of $0.26 \mathrm{~ms}$. This effect was statistically significant $(\mathrm{P}=0.01 ; \mathrm{n}=9)$ with the Wilcoxon signed rank test. All other components showed both increases and decreases in latency and these changes were not significant. No significant change was observed in the amplitude of any component.

Changes in latency of the $\mathrm{A}_{\text {ind }}$ component are plotted in relation to changes in heart rate and contractility caused by burst stimulation and dobutamine in figure 3.9 .

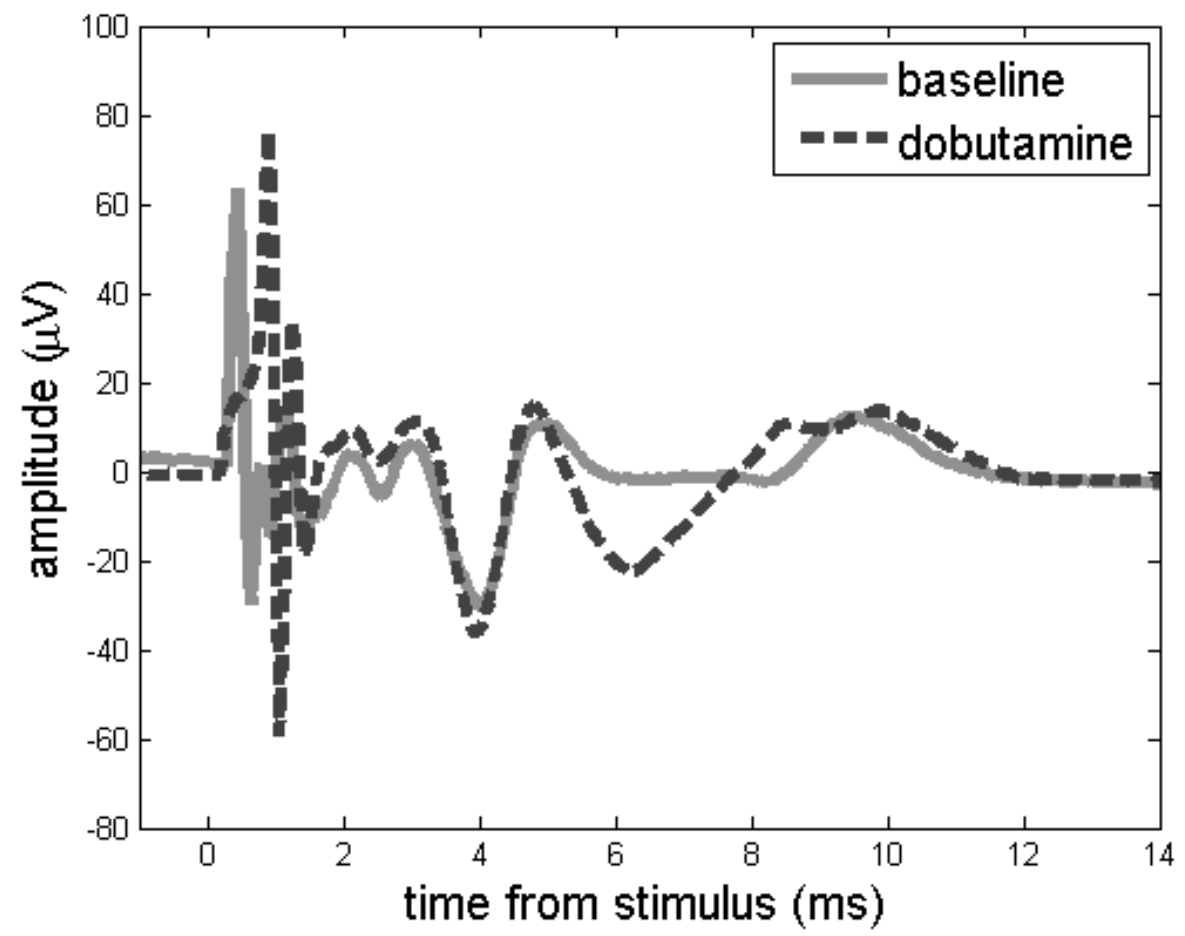

Figure 3.8: Example of the effect of the administration of dobutamine on the CAP. A decrease in latency of the $A_{\text {ind }}$ component (which occurs around $t=8 m$ s in this example) was found for all pigs $(n=9)$. The stimulus artefact $(a t=0)$ has been removed from the plot by subtracting a template. Example from pig 3. 


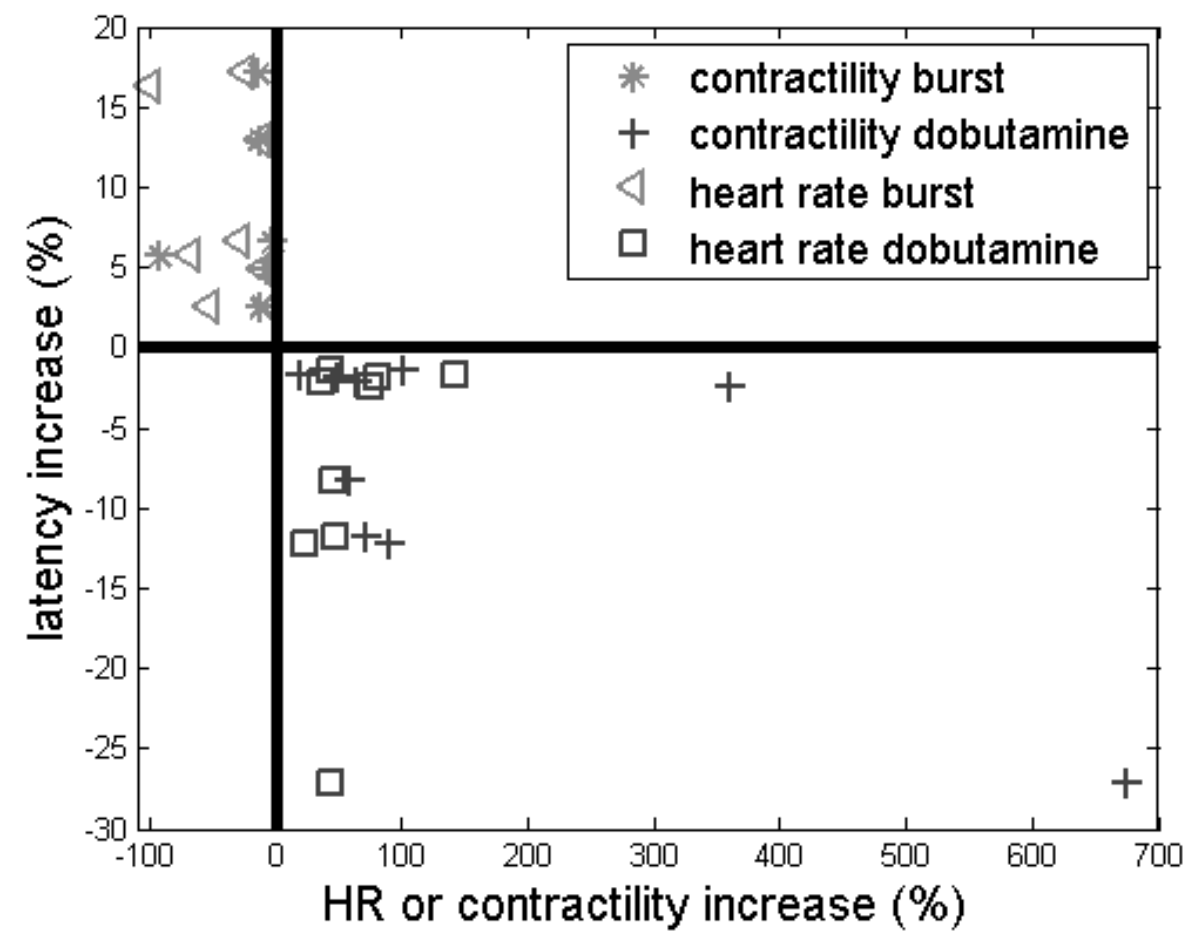

Figure 3.9: Overview of how latency of the $A_{\text {ind }}$ component changes when cardiac parameters heart rate and contractility change. Symbols indicate change in contractility during burst $\left({ }^{*} ; n=6\right)$ and dobutamine $(+; n=9)$, and change in heart rate during burst $(\triangleleft ; n=7)$ and dobutamine $\square ; n=9)$. In all pigs, when heart rate and contractility were decreased due to burst stimulation, latency of the $A_{\text {ind }}$ component was increased $(\triangleleft$ and $*)$. When heart rate and contractility were increased due to dobutamine, latency of the $A_{\text {ind }}$ component was decreased in all pigs $(\square$ and +$)$.

\subsection{Discussion}

In this chapter the evoked CAP in the cervical vagus nerve was studied. The first question addressed was whether an indirect component from the periphery or an indirect component from the brain stem could be found.

A component was found that had a low threshold, a high velocity, came from the direction of the periphery and had a long latency. The latency of this component does not match the low threshold and high velocity, which shows that this component is an indirect component. It was called the $\mathrm{A}_{\text {ind }}$ component. When the left vagus nerve is stimulated, the $\mathrm{A}_{\text {ind }}$ component occurs on both the left and the right vagus nerve. The $\mathrm{A}_{\text {ind }}$ component shows a difference in shape and latency in recordings from different electrode sites, which excludes the possibility that it is a far field potential. When electrical stimulation was 
repeated after the nerve was cut caudal to the electrodes in two pigs, the $A_{\text {ind }}$ component had disappeared. This supports the conclusion that the $\mathrm{A}_{\text {ind }}$ component is not a result of direct stimulation. Based on these results, it can be concluded that there is an indirect component in the evoked CAP, coming from the periphery.

The threshold of the $\mathrm{A}_{\text {ind }}$ component was in the range of thick myelinated fibres, showing that these are the fibres that constitute the efferent pathway that resulted in the $A_{\text {ind }}$ response. The threshold was close to that of the $A \alpha$ fibres $\left(0.16 \pm 0.13 \mathrm{~mA}\right.$ for the $\mathrm{A}_{\text {ind }}$ component and $0.13 \pm 0.08 \mathrm{~mA}$ for the $\mathrm{A} \alpha$ component), suggesting involvement of a fibre type in the low range of $A \alpha$ fibre diameter or the high range of $\mathrm{A} \beta$ fibre diameter. In other words, the efferent pathway of the $\mathrm{A}_{\text {ind }}$ component comprises fibres with a diameter of around $12 \mu \mathrm{m}$. This means that cardiac efferent fibres may be involved, since cardiac efferent and afferent vagal fibres range from $1-12 \mu \mathrm{m}$ in diameter [2]. The velocity of the $A_{\text {ind }}$ component along the nerve gives an indication of the fibre type involved in the afferent limb. Using the approximation that the fibre diameter in $\mu \mathrm{m}$ equals one-sixth of the conduction velocity in $\mathrm{m} / \mathrm{s}$ [9], the velocity of $69 \pm 17 \mathrm{~m} / \mathrm{s}$ leads to an estimated fibre diameter of about $11 \mu \mathrm{m}$. Again, this is within the range of cardiac vagal fibres.

A latency of $8.13 \pm 1.12 \mathrm{~ms}$ was found for the $\mathrm{A}_{\text {ind }}$ component. The length of the vagus nerve from stimulation site to the heart was not measured in the experiments, so we can only give a rough estimate on the feasibility of this latency with the involvement of the neural network on the heart. Roughly estimating the distance between the electrode cuffs and the heart at $25 \mathrm{~cm}$, it would take approximately $3.5 \mathrm{~ms}$ for the efferent limb to reach the heart and $3.6 \mathrm{~ms}$ for the resulting component to go from the heart to the cuffs. This would lead to a total latency of about $7.1 \mathrm{~ms}$, which is quite close to the latency found. So the latency we found also matches a hypothetical response from the neural network on the heart.

In the four pigs in which an additional electrode cuff was placed on the right vagus nerve, the $A_{\text {ind }}$ component was visible on both the left and right vagus nerve when the left vagus nerve was stimulated. The $A \alpha, A \beta$ and $B$ components of the CAP did not occur on the right vagus nerve when the left vagus nerve was stimulated. Consequently, it can be concluded that the $\mathrm{A}_{\text {ind }}$ component is conducted through both the left and the right vagus nerve as a result of onesided vagus nerve stimulation.

The latency of the $\mathrm{A}_{\text {ind }}$ component was not identical on both nerves, which can be caused by differences in distance from the heart. The shape also differed, which may be caused by differences in fibre distribution or dispersion.

The $\mathrm{A}_{\text {ind }}$ response has not been reported in the literature before, even though the vagal CAP has been studied. This may be because of the use of a tripolar 
derivation in other studies. Since the indirect component is dispersed and fast, the second order temporal and spatial derivatives are relatively small. Therefore the tripolar derivation of the signal is also relatively small, in contrast to the average reference derivation we used. We showed this in an earlier publication by calculating the tripolar derivation from the average reference recording [10]. Therefore, it may have been missed in other studies due to the use of a tripolar derivation, as may for example have been the case in [11].

Alternatively, it may have been misclassified as a slow response of $\mathrm{C}$ fibres in other studies. For example, in a study by Evans et al. [12], who used a bipolar hook electrode to record the CAPs in humans, a C-fibre response was found. In two out of four patients, the response they classified as coming from $\mathrm{C}$ fibres based on latency, was observed at the smallest stimulus amplitude $(0.25 \mathrm{~mA})$ and increased little with an increase in stimulus current. This low threshold does not match a $\mathrm{C}$ fibre response and therefore it may have been an $\mathrm{A}_{\text {ind }}$ response.

An indirect component coming from the brain stem was not found. No components were observed that travelled in the direction of the periphery, and no components disappeared when the nerve was cut rostral to the electrodes. The hypothesized indirect component coming from the brain stem would have been the result of the baroreceptor reflex. Fibres from aortic arch baroreceptors constitute the afferent limb of the reflex. These afferent fibres mainly consist of fibres with a diameter of $4 \mu \mathrm{m}$ or $10 \mu \mathrm{m}$ [2]. Fibres of this diameter and smaller have been activated in our experiments, which shows that a lack of stimulation current intensity was not the reason why we did not find an indirect component coming from the brain stem. The component we expected to record (the efferent limb) would come from small diameter myelinated fibres [13]. The amplitude of these fibres is small, which may be the reason why we did not detect an indirect component coming from the brainstem.

Another interpretation is that electrical stimulation of the cervical vagus nerve does not lead to a reflexive efferent response. Alternatively, anaesthetics may have repressed the cardiac reflexes [14].

No response from $\mathrm{C}$ fibres was observed, even though these unmyelinated fibres are present in the cervical vagus nerve [2]. Stimulation amplitude may have been too low to evoke a $\mathrm{C}$ fibre response [15]. As an example, Tosato et al.[11], who used an experimental setup similar to ours, found $\mathrm{C}$ fibre responses at stimulation amplitudes of at least $9 \mathrm{~mA}$. This is much higher than the $5 \mathrm{~mA}$ we used in this study. The amplitude of $5 \mathrm{~mA}$ was high enough to affect the heart, which shows that relevant cardiac vagal fibres were activated. Higher amplitudes may be used in future studies, to obtain a more complete picture of the vagal CAP.

The $\mathrm{A}_{\text {ind }}$ component has a latency that is similar to the latency of a $\mathrm{C}$ fibre response, but stimulation threshold and velocity show that it does not originate from $\mathrm{C}$ fibres. The stimulation threshold of the $\mathrm{A}_{\text {ind }}$ component is $0.16 \pm$ 
$0.13 \mathrm{~mA}$, while the threshold for $\mathrm{C}$ fibres in this setup would be at least $9 \mathrm{~mA}$ [11]. The $A_{\text {ind }}$ component has a velocity of $69 \pm 17 \mathrm{~m} / \mathrm{s}$, while $C$ fibres have a velocity of less than $2 \mathrm{~m} / \mathrm{s}$ [1].

The second question addressed in this chapter was whether the indirect component depends on the state of the cardiovascular system. To assess this, latency and amplitude of the $\mathrm{A}_{\text {ind }}$ component was compared between baseline, decreased heart rate and contractility caused by burst stimulation, and increased heart rate and contractility caused by dobutamine. Latency of the $\mathrm{A}_{\text {ind }}$ component was increased when heart rate and contractility were lowered by burst stimulation. The CAPs recorded during increased heart rate and contractility caused by dobutamine consistently showed a decreased latency of the $\mathrm{A}_{\text {ind }}$ component. This may indicate that changes in the loop via the intrinsic cardiac network lead to a change in relay time of the indirect component, which results in a change in latency of the $\mathrm{A}_{\text {ind }}$ component.

However, it can not be excluded with the current experimental setup that influences from other organs are a cause. Since the gastrointestinal tract has an intrinsic nervous system [16], an indirect component from the periphery could also originate there. However, vagal abdominal fibres have a diameter of less than $6 \mu \mathrm{m}$ [2], which does not match the threshold, velocity and latency of our $\mathrm{A}_{\text {ind }}$ component. To the best of our knowledge, no other organs that are innervated by the vagus nerve have an intrinsic nervous system. The lungs, larynx, and the oesophagus are other organs that are innervated by the vagus nerve and whose fibres are present at the cervical level. Possible involvement of the lungs in the $\mathrm{A}_{\text {ind }}$ component is ruled out, since efferent bronchial fibres have a diameter of less than $4 \mu \mathrm{m}$ [2]. A response coming from these fibres would have a much higher threshold. The larynx also cannot be involved, since it has little to no afferent vagal innervation [17]. Afferent fibres of the oesophagus are no thicker than $\mathrm{C}$ or $\mathrm{A} \delta$ fibres [18], which also have a lower velocity than found for the $\mathrm{A}_{\text {ind }}$ component. This leaves the heart as the most likely source of the $\mathrm{A}_{\text {ind }}$ component.

It is known that repetitive burst stimulation itself can lead to an increased latency of CAPs, due to hyperpolarisation of the membranes [19]. However, it is unlikely that this was the cause of the increased latency of the $\mathrm{A}_{\text {ind }}$ component, since myelinated fibres do not typically show such activity dependent changes [20] and a shift in latency occurs in neither the A $\alpha$ nor the $\mathrm{A} \beta$ component. Another thing to consider is that stimulation in the analyzed recordings was performed at a level substantially above fibre threshold. This means that variations in recruitment characteristics - which can occur around fibre threshold - also can not have caused the observed changes.

Changes in $\mathrm{A} \alpha, \mathrm{A} \beta$ and $\mathrm{B}$ components were also observed in the recordings with dobutamine added, but were neither consistent nor significant. These changes may be due to changes that occurred in time, since the recordings at 
baseline condition and with dobutamine were made more than an hour apart in most pigs.

Although our results suggest that the $\mathrm{A}_{\text {ind }}$ component has a cardiovascular origin, at this point there is no undisputable evidence that the $\mathrm{A}_{\text {ind }}$ component is related to the cardiovascular system. The effects of changes in the state of the cardiovascular system on the $\mathrm{A}_{\text {ind }}$ component need to be further examined. Influences of other organs on the vagus nerve should be excluded by blocking or cutting all non-cardiac vagal branches. Alternatively, pharmacological blockage of selected vagal cardiac branches via atropine administration or baroreflex denervation may be used to study the effect of these interventions on the evoked components.

The link with pathological conditions is of special interest, because of the clinical applications it provides. For example, arrhythmias and ischemia can influence the intrinsic cardiac nervous system [21], which may lead to changes in the indirect component. Changes in the neurocardiac system have been related to pathological conditions such as ischemia, arrhythmias and congestive heart failure [22-25]. Monitoring the neurocardiac system to detect these events has many potential clinical applications. We propose to use the evoked CAP of the cervical vagus nerve as an indicator of the neurocardiac system.

\subsection{Conclusions}

An indirect response occurs in both the left and right vagus nerve when stimulating at the cervical level. This indirect component originates in the periphery. Possibly, it is transmitted via the neural network on the heart.

With decreased heart rate and contractility, caused by burst stimulation, the $\mathrm{A}_{\text {ind }}$ component had a longer latency. When the cardiovascular system is influenced by using dobutamine, the $\mathrm{A}_{\text {ind }}$ component had a shorter latency. The latency of the $\mathrm{A}_{\text {ind }}$ component of the CAP may be related to the state of the cardiovascular system. This needs to be further explored.

\section{References}

1. Robinson, A.J. and Snyder-Mackler, L. (2008) Clinical electrophysiology : electrotherapy and electrophysiologic testing 3rd ed Philadelphia Wolters Kluwer/Lippincott Williams \& Wilkins

2. Agostoni, E., Chinnock, J.E., Daly, M.D.B., and Murray J.G. (1957) Functional and Histological Studies of the Vagus Nerve and Its Branches to the Heart, Lungs and Abdominal Viscera in the Cat $\mathrm{J}$ Physiol-London, 135(1), p. 182-205

3. Armour, J.A. (2007) The little brain on the heart Clev Clin J Med 74(Supplement 1), p. S48-S51

4. Ludbrook, J. (1990) Cardiovascular reflexes from cardiac sensory receptors Aust NZ J Med, 20(4), p. 597-606 
5. Kollai, M. and Koizumi, K. (1979) Reciprocal and Non-Reciprocal Action of the Vagal and Sympathetic-Nerves Innervating the Heart $\mathbf{J}$ Autonom Nerv Syst 1(1), p. 33-52

6. Mravec, B., Ondicova, K., Valaskova, Z., Gidron, Y., and Hulin, I. (2009) Neurobiological principles in the etiopathogenesis of disease when diseases have a head Med Sci Monit 15(1), p. RA6-16

7. Offner, F.F. (1950) The EEG as Potential Mapping - the Value of the Average Monopolar Reference Electroen Clin Neuro 2(2), p. 213-4

8. Randall, D.C. (1974) Concurrent Measurement of Left-Ventricular DpDtmax, Isometric Contractile-Force and Cardiac Loading in Intact Monkey Cardiology 59(5), p. 304-18

9. Hursh, J.B. (1939) Conduction velocity and diameter of nerve fibres Am J Physiol 127, p. 131-9

10. Ordelman, S.C.M.A., Kornet, L., Cornelussen, R., Buschman, H.P.J., and Veltink, P.H. Average reference recording from the vagus nerve reveals an evoked indirect response 4th Int. IEEE EMBS Conf on Neural Engineering 2009 Antalya

11. Tosato, M., Yoshida, K., Toft, E., Nekrasas, V., and Struijk, J.J. (2006) Closed-loop control of the heart rate by electrical stimulation of the vagus nerve Med Biol Eng Comput 44(3), p. 161-9

12. Evans, M.S., Verma-Ahuja, S., Naritoku, D.K., and Espinosa, J.A. (2004) Intraoperative human vagus nerve compound action potentials Acta Neurol Scand 110(4), p. 232-8

13. Kunze, D.L. (1972) Reflex Discharge Patterns of Cardiac Vagal Efferent Fibres J Physiol-London 222(1), p. 1-15

14. Urban, B.W. (2002) Current assessment of targets and theories of anaesthesia Brit J Anaesth 89(1), p. 167-83

15. Middleton, S., Middleton, H.H., and Grundfest, H. (1950) Spike potentials and cardiac effects of mammalian vagus nerve Am J Physiol 162(3), p. 545-52

16. Travagli, R.A., Hermann, G.E., Browning, K.N., and Rogers, R.C. (2006) Brainstem circuits regulating gastric function Annu Rev Physiol 68, p. 279-305

17. Murray, J.G. (1957) Innervation of the intrinsic muscles of the cat's larynx by the recurrent laryngeal nerve: a unimodal nerve J Physiol 135(1), p. 206-12

18. Lennerz, J.K., Dentsch, C., Bernardini, N., Hummel, T., Neuhuber, W.L., and Reeh, P.W. (2007) Electrophysiological characterization of vagal afferents relevant to mucosal nociception in the rat upper oesophagus J Physiol 582(Pt 1), p. 229-42

19. Bostock, H. and Grafe, P. (1985) Activity-Dependent Excitability Changes in Normal and Demyelinated Rat Spinal Root Axons J PhysiolLondon 365(Aug), p. 239-57 
20. Grafe, P., Quasthoff, S., Grosskreutz, J., and Alzheimer, C. (1997) Function of the hyperpolarization-activated inward rectification in nonmyelinated peripheral rat and human axons J Neurophysiol 77(1), p. 421-6

21. Armour, J.A. (2008) Potential clinical relevance of the 'little brain' on the mammalian heart Exp Physiol 93(2), p. 165-76

22. Gilmore, J.P. and Zucker, I.H. (1974) Discharge of Type-B Atrial Receptors during Changes in Vascular Volume and Depression of Atrial Contractility J Physiol-London 239(2), p. 207-23

23. Greenberg, T.T., Richmond, W.H., Stocking, R.A., Gupta, P.D., Meehan, J.P., and Henry, J.P. (1973) Impaired Atrial Receptor Responses in Dogs with Heart-Failure Due to Tricuspid Insufficiency and Pulmonary-Artery Stenosis Circ Res 33(4), p. 424-33

24. Zucker, I.H. and Gilmore, J.P. (1973) Left Atrial Receptor Discharge during Atrial Arrhythmias in Dog Circ Res 33(6), p. 672-7

25. Chen, R.P.C., Thompson, G.W., and Armour, J.A. (2001) Transduction capabilities of neonatal ventricular afferent neurons in vivo Auton Neurosci-Basic 87(1), p. 1-8 


\section{Chapter 4}

\section{The average reference method for recording a dispersed evoked signal from peripheral nerves - a modelling study}

Simone Ordelman Lilian Kornet Peter Veltink 


\section{Abstract}

In this chapter a computer model is used to recreate a dispersed component that was previously observed in the evoked compound action potential on the pig vagus nerve. It is hypothesized that this dispersed component is the result of a summation of single fibre action potentials that have varying latencies. In the animal experiments, the average reference method was used for recording, and the dispersed component was strongly reduced on the centre electrode in a cuff with three rings.

A summation of 500 simulated single fibre action potentials with varying latencies resulted in a dispersed component if their arrival times in the cuff were non-random. The width and amplitude of the dispersed component could be influenced by the number of fibres, arrival time pattern and fibre diameter range. The simulated dispersed component was observed to a similar extent on all three electrodes in a cuff with three rings.

The occurrence of a dispersed, fast neural component can be explained by the hypothesized physiological origin of a dispersed burst of single fibre action potentials. The fact that the simulated dispersed component was similar on the centre electrode and the outer electrodes using the computer model does not support this hypothesis. 


\subsection{Introduction}

We have analyzed the evoked compound action potential (CAP) of the vagus nerve in previous animal experiments [1]. A component was found in the evoked CAP that had a high velocity along the nerve but a long latency, from which we concluded that this was an indirect component [1]. It came from the periphery, i.e. it emanated from an afferent pathway. We hypothesized that since the vagus nerve is involved in reflexive control, electrical stimulation of the vagus nerve may result in indirect components relating to reflexive loops. For example, the baroreceptor reflex is a reflexive loop that possibly leads to indirect components when electrically stimulating the vagus nerve, either from the brain stem (the efferent path) or from the heart (the afferent path).

Because of its high velocity along the nerve, we hypothesized that the indirect component is a result of a summation of single fibre action potentials from thick, myelinated fibres.

In the animal experiments, the velocity found along the nerve was about $70 \mathrm{~m} / \mathrm{s}$ [1]. The approximation that the fibre diameter in $\mu \mathrm{m}$ equals one-sixth of the conduction velocity in $\mathrm{m} / \mathrm{s}$ [2] led to an estimated fibre diameter of about $11 \mu \mathrm{m}$. This is within the range of cardiac vagal fibre diameters, which have been estimated between 1 and $12 \mu \mathrm{m}$ [3]. The indirect component had a dispersed shape, which we interpreted as it being a component that resulted from a summation of action potentials with varying delays.

The average reference method [4] was used for recording in our animal experiments, which uses the average of all recorded channels and subtracts this from each channel separately. It is not yet commonly used for peripheral nerve recording, in contrast to a tripolar configuration, in which the average signal of the two outer electrodes are subtracted from the centre electrode. We chose to use the average reference method, because it allows signals to be recorded from each electrode site on the cuff separately, instead of one channel from each three electrodes as with a tripolar configuration. This is especially suitable for improving spatial selectivity, or for multiple-electrode configurations such as proposed by [5] for velocity-selective filtering. It allows direction and velocity of signals to be determined within a multiple electrode cuff, by dividing the time it takes for a component of the CAP to go from one electrode to the next by the distance between the two electrodes. This could also be achieved with multiple tripolar configurations, but would then require more electrodes. Space along the nerve is generally limited by anatomical constraints, which makes the average reference method a better choice because fewer electrodes, thus smaller cuff length is needed for velocity and direction measurements.

The component was strongly reduced with a tripolar derivation and on the centre electrode in the average reference configuration, possibly because this 
configuration records the second order spatial derivative. A dispersed shape that has a close to linear field in the cuff will be strongly reduced with such a derivation.

In the current chapter, a computer model of single fibre action potentials is used to test our hypotheses on the physiological origin of the indirect component and to seek an explanation for the difference found between the signals recorded with the three electrodes in an average reference configuration in the animal experiments. The fibre model by Wesselink [6] was chosen, because of its accurate fit to human nerve fibre characteristics.

\subsection{Methods}

\subsubsection{The computer model}

A myelinated nerve fibre can be modelled as point current sources, located at the nodes of Ranvier $[5,13,18]$. To simulate action potentials of a myelinated nerve, the model of Wesselink, Holsheimer and Boom is used in this chapter [6]. The main equations and parameters of this model are described in the Appendix. To generate an action potential, a voltage field that would result from a monophasic stimulus of amplitude $5 \mathrm{~mA}$ and pulse width $300 \mu$ s was added at one end of the modelled nerve, $2 \mathrm{~cm}$ from the cuff's beginning. The nodal currents were calculated by integrating the differential equations using the Euler method with a step size of $1 \mu \mathrm{s}$.

The signal recorded by the cuff electrode can be calculated as the sum of the signals from each current source inside the cuff [7]. A one-dimensional model can be used, when assuming that the intracellular and extracellular currents flow only in axial directions inside the cuff. The one-dimensional model has been shown to be a good approximation of a three-dimensional model [8-9]. A weight function $\mathrm{w}(\mathrm{x})$ can be used to calculate the contribution from a current source to the recorded signal [7]. The single fibre action potential (SFAP) that is recorded as a result of an action current from a nerve fibre in the cuff can be calculated as follows:

$$
\operatorname{SFAP}(t)=\sum_{n=1}^{N n} I_{a c}\left(x_{n}, t\right) w\left(x_{n}\right)
$$

Where $I_{a c}\left(x_{n}, t\right)$ is the action current at a position $x_{n}$ at time $t$, with $n$ the node number. $\mathrm{w}\left(\mathrm{x}_{\mathrm{n}}\right)$ is the weight function at position $\mathrm{x}_{\mathrm{n}}$. $\mathrm{Nn}$ is the number of nodes modelled, which is equal to the nerve length divided by the length of the internode plus the node length. Length of the nerve $\left(\mathrm{L}_{\text {nerve }}\right)$ can be chosen as any length long enough to accommodate placement of a nerve cuff. Length of the 
internode $\left(\mathrm{L}_{\text {internode }}\right)$ depends linearly on fibre diameter $(100 * \mathrm{D})$ [6-7,13-14], while node length $\left(\mathrm{L}_{\text {node }}\right)$ is constant at $1.5 \mu \mathrm{m}[8]$ :

$\mathrm{Nn}=\frac{\mathrm{L}_{\text {nerve }}}{\mathrm{L}_{\text {internode }}+\mathrm{L}_{\text {node }}}$

The weight function depends on cuff dimensions and electrode position [7, 10]. To determine the weight function of a tripolar or average reference derivation, first the weight function of a monopolar electrode configuration is determined. In case of a monopolar configuration, the weight function can be calculated by applying the reciprocity theorem [11], assuming the injection of a current at the electrode (figure 4.1b).

a.

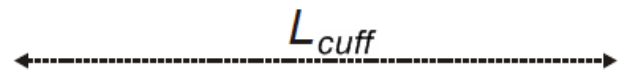

\section{electrode}

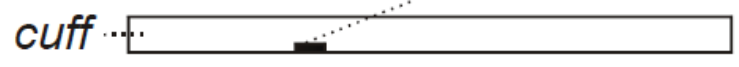

nerve

$b$.

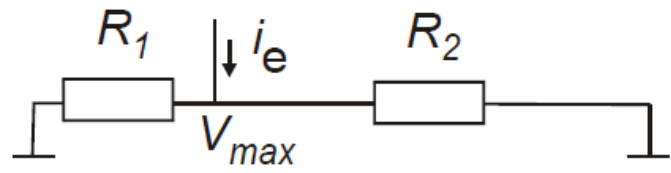

C.

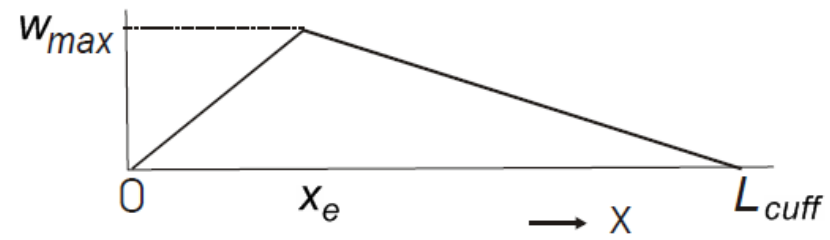

Figure 4.1: a. A monopolar cuff electrode. $b$. Effective resistive loading of electrode at position $x_{e}$ if a current $i_{e}$ were injected at this electrode. $\mathrm{R}_{1}=\frac{\mathrm{x}_{\mathrm{e}}}{\pi * \mathrm{r}_{\mathrm{cuff}}^{2} * \sigma_{\mathrm{z}}}$ and $\mathrm{R}_{2}=\frac{\mathrm{L}_{\text {cuff }}-\mathrm{x}_{\mathrm{e}}}{\pi * \mathrm{r}_{\mathrm{cuff}}^{2} * \sigma_{\mathrm{z}}}$, with parameters as in equation (4.6) c. Resulting calculated weight function. Adapted from [9] 
Since we assumed a one-dimensional conduction within the cuff and a single specific longitudinal conductivity of the tissue within the cuff, $\mathrm{w}\left(\mathrm{x}_{\mathrm{n}}\right)$ follows (figure 4.1c) $[3,13,17]$ :

$\mathrm{w}(\mathrm{x})=\mathrm{w}_{\text {max }} \mathrm{f}(\mathrm{x})$

With

$\mathrm{w}_{\text {max }}=\mathrm{R}_{\mathrm{e}} \frac{\mathrm{x}_{\mathrm{e}}\left(\mathrm{L}_{\text {cuff }}-\mathrm{x}_{\mathrm{e}}\right)}{\mathrm{L}_{\text {cuff }}}$

Where $\mathrm{L}_{\text {cuff }}$ is the length of the cuff, $\mathrm{R}_{\mathrm{e}}$ the longitudinal resistance in the cuff, $\mathrm{x}$ the position along the longitudinal axis of the cuff and $\mathrm{x}_{\mathrm{e}}$ the electrode position.

$$
\begin{aligned}
& f(x)=\frac{x}{x_{e}} \text { for } 0<x<x_{e} \\
& f(x)=\frac{L_{\text {cuff }}-x}{L_{\text {cuff }}-x_{e}} \text { for } x_{e}<x<L_{\text {cuff }}
\end{aligned}
$$

$\mathrm{R}_{\mathrm{e}}$ can be calculated as follows:

$$
\mathrm{R}_{\mathrm{e}}=\frac{\mathrm{L}_{\text {cuff }}}{\pi * \mathrm{r}_{\text {cuff }}^{2} * \sigma_{\mathrm{z}}}
$$

With $r_{\text {cuff }}$ the radius of the cuff and $\sigma_{z}$ the axial conductivity set at 1 (ohm.m) $)^{-1}$ [8].

The monopolar weight functions were used to calculate the weight function for the average reference configuration (4.7) and the tripolar configuration (4.8):

$$
\begin{aligned}
& \mathrm{w}_{\mathrm{ar}, \mathrm{k}}=\mathrm{w}_{\mathrm{k}}-\frac{1}{\mathrm{n}_{\mathrm{e}}} \sum_{\mathrm{i}=1}^{\mathrm{n}_{\mathrm{e}}} \mathrm{w}_{\mathrm{i}}, \quad \mathrm{k}=1 \ldots \mathrm{n}_{\mathrm{e}} \\
& \mathrm{w}_{\text {trip }}=\mathrm{w}_{2}-\frac{1}{2} \mathrm{w}_{1}-\frac{1}{2} \mathrm{w}_{3}
\end{aligned}
$$

with $n_{e}$ the number of electrodes on the cuff, $w_{a r, k}$ the weight function for electrode $\mathrm{k}$ of the average reference method, $\mathrm{w}_{\mathrm{k}}$ and $\mathrm{w}_{\mathrm{i}}$ the weight function for the monopolar electrode number $\mathrm{k}$ and $\mathrm{i}$ respectively. With these weight functions it can be shown that the only difference between the tripolar derivation and the centre electrode in the average reference configuration is a factor $2 / 3$.

$$
w_{a r, 2}=w_{2}-\frac{1}{3} \sum_{i=1}^{3} w_{i}=\frac{2}{3} w_{2}-\frac{1}{3} w_{1}-\frac{1}{3} w_{3}=\frac{2}{3} w_{\text {trip }}
$$




\subsubsection{Simulation protocol}

For all simulations, fibre diameter was $11 \mu \mathrm{m}$, cuff length was $15 \mathrm{~mm}$, cuff radius was $1 \mathrm{~mm}$ and electrode positions were $3.5 \mathrm{~mm}, 7.5 \mathrm{~mm}$ and $11.5 \mathrm{~mm}$ respectively from the cuff's end.

The recorded signals were compared between the three electrodes of the average reference configuration. To compare the shape of the SFAP between the electrodes, the maximum to minimum peak-to-peak recorded amplitude $\left(\mathrm{V}_{\mathrm{pp}}\right)$, the number of phases and the duration of the SFAP were analyzed.

The indirect evoked component was modelled from a summation of SFAPs that entered the cuff within a small time window, but not simultaneously. We used an inhomogeneous Poisson process with varying values for $\lambda$, to determine the delays. A Poisson process describes the distribution of arrivals, in our case the arrival of a SFAP in the cuff. In a Poisson process, the wait time for the next arrival has an exponential probability distribution function:

$$
F(t)=1-e^{-\lambda t}
$$

with $\lambda$ the arrival rate.

Using (4.10), the time to the next arrival can be calculated, with $u$ defined as a random variable from a uniform distribution between 0 and 1 :

$$
\mathrm{t}=-\frac{1}{\lambda} \ln (\mathrm{u})
$$

Due to the memoryless property of the Poisson process the time to each next arrival can be calculated with (4.11), independent of previous arrivals. The arrival rate $\lambda$ was calculated by dividing the expected number of action potentials by the time window length during which they occur. The number of SFAPs was set at 500 , based on the number of fibres in the vagus nerve that have a diameter of $10 \mu \mathrm{m}$ or larger [3]. The total time in which the SFAPs entered the cuff $\left(\mathrm{t}_{\max }\right)$ was set at $5 \mathrm{~ms}$. During this time window, $\lambda$ was increased and decreased:

$$
\begin{aligned}
& \mathrm{f}(\mathrm{t})=\mathrm{e}^{-\left(\frac{\mathrm{t}-0.5 * \mathrm{t}_{\max }}{\tau}\right)^{2}} \\
& \lambda(\mathrm{t})=\mathrm{f}(\mathrm{t}) * \mathrm{~K}(\tau)
\end{aligned}
$$

With $\mathrm{f}$ a bell-shaped function with a maximum at $0.5 * \mathrm{t}_{\max }$. This function is scaled in (4.13) with factor $K$ to yield a bell-shaped pattern of $\lambda$ that results in the above mentioned total number of SFAPs. The $\lambda$ pattern was varied by varying time constant $\tau$ between $1 \mathrm{~ms}$ and $\mathrm{t}_{\max }$. The value for the next arrival 
time was either calculated as $1 / \lambda$, or in a random condition using (4.11). The effects of the $\lambda$ patterns and the recording configuration on the dispersed component's shape (number of phases and width), and amplitude $\left(\mathrm{V}_{\mathrm{pp}}\right)$ were analyzed.

\subsection{Results}

Integration of the differential equations described in the appendix resulted in nodal currents and transmembrane potentials, an example of which is shown for an $11 \mu \mathrm{m}$ fibre in figure 4.2, which corresponds with the result shown by Wesselink [6].
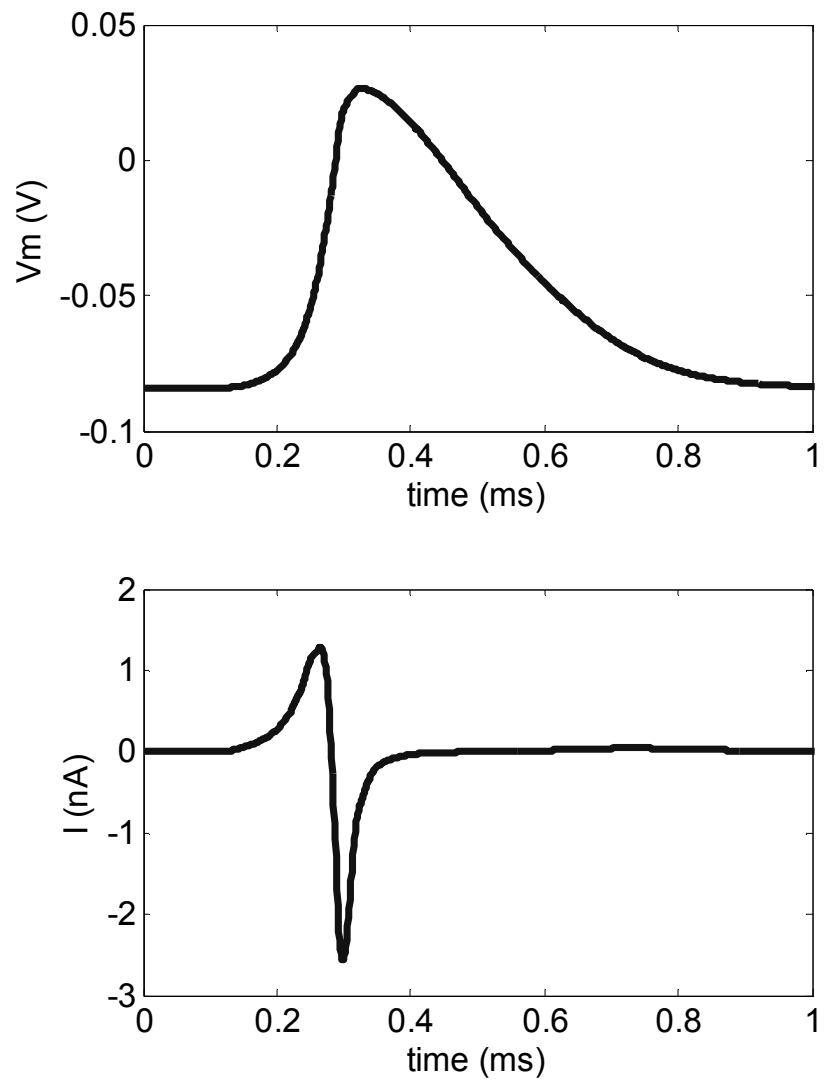

Figure 4.2: Transmembrane potential (top) and action current (bottom) for a fibre with a diameter of $11 \mu \mathrm{m}$. Example from a node located 90 internodal distances from the stimulus. 
The shape of a recorded SFAP vary between the electrodes of the average reference method (figure 4.3). The SFAPs recorded with the outer electrodes in the average reference method have four phases instead of three with the centre electrode. This is reflected in their weight functions by the extra phase (figure 4.3). The SFAPs had a width of $0.4 \mathrm{~ms}, 0.3 \mathrm{~ms}$ and $0.4 \mathrm{~ms}$ for the three electrodes respectively. The $\mathrm{V}_{\mathrm{pp}}$ values are also comparable between the three electrodes, at $0.033 \mu \mathrm{V}, 0.030 \mu \mathrm{V}$ and $0.030 \mu \mathrm{V}$ respectively.
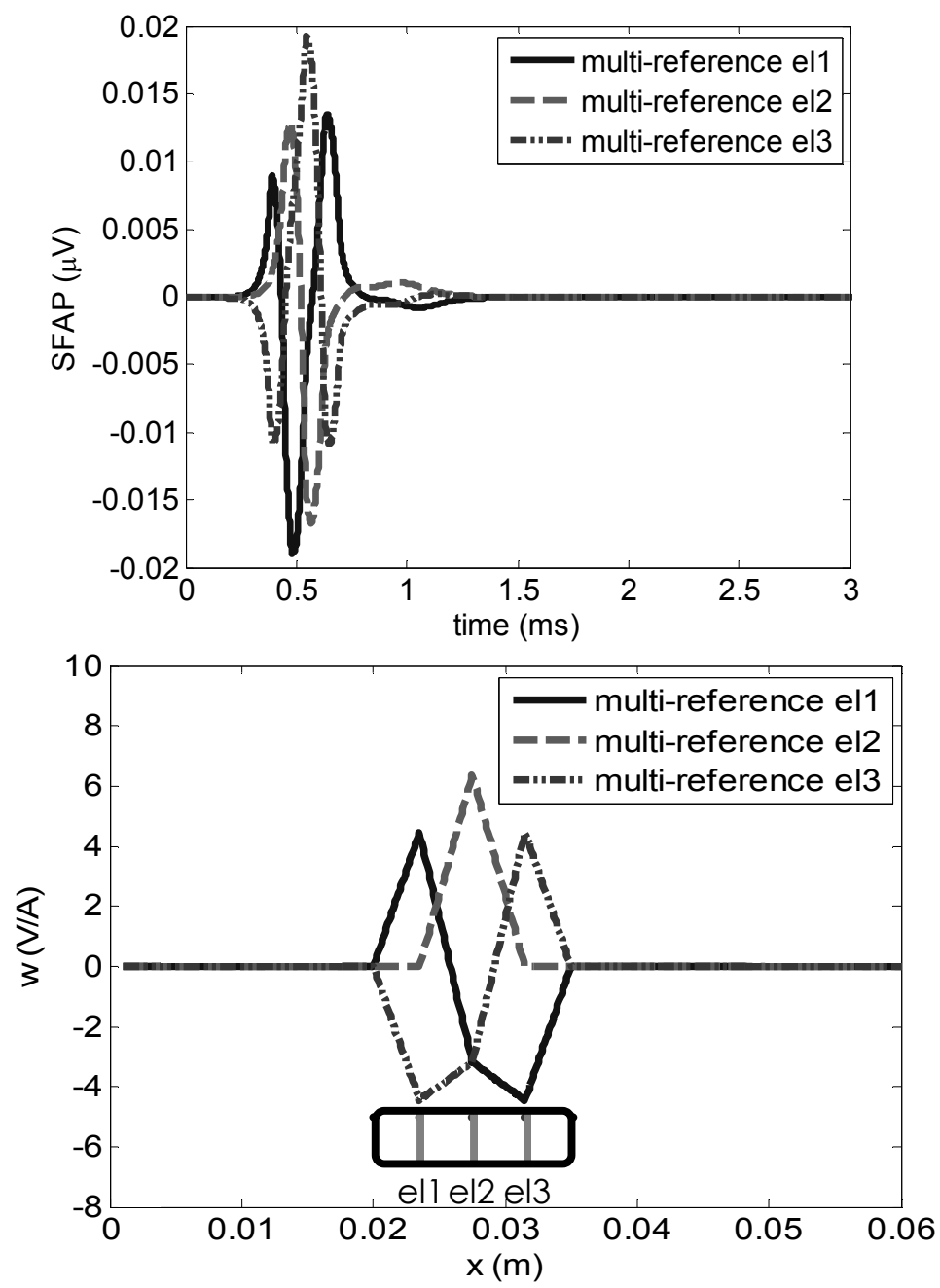

Figure 4.3: SFAPS (top) and weight functions (bottom) for the average reference method. The cuff is shown with the weight functions for reference. Fibre diameter was $11 \mu \mathrm{m}$, cuff length was $15 \mathrm{~mm}$, cuff radius was $1 \mathrm{~mm}$ and electrode positions were 3.5 $\mathrm{mm}, 7.5 \mathrm{~mm}$ and $11.5 \mathrm{~mm}$ respectively from the cuff's end. At $t=0$ the nerve was stimulated at $x=0$, which is $2 \mathrm{~cm}$ from the cuff electrode. 

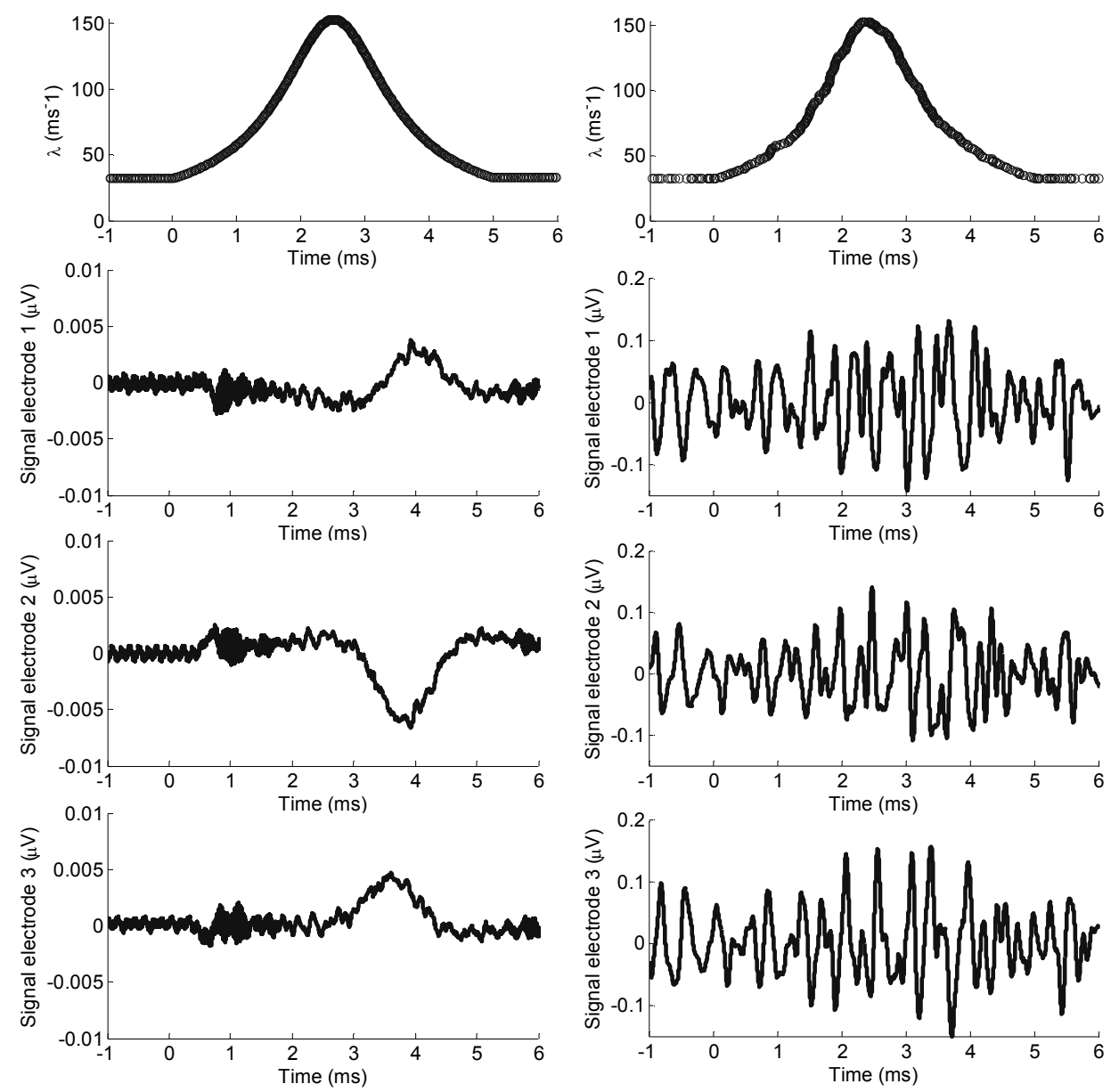

Figure 4.4: Simulating the dispersed component in the non-random condition (left column) and the random condition (right column). Top row: The change in rate parameter $\lambda$ during the time window of $5 \mathrm{~ms}$, with $\tau=4 \mathrm{~ms}$. Bottom three rows: The recorded CAP with $\lambda$ pattern as in the top row, with the centre electrode (electrode 2) and the two outer electrodes (electrode 1 and electrode 3) of the average reference configuration. 
A dispersed indirect component of an evoked CAP was modelled. Figure 4.4 shows an example of the simulated component for both the centre electrode and the outer electrodes of the average reference configuration. The recorded component varies little between the electrodes. In the non-random condition, when the arrival rate $\lambda$ was increased and decreased during the time window, creating a bell shape (figure 4.4), the recorded component showed a dispersed shape around the centre of the time window (figure 4.4), which is similar to the animal experimental results (figure 4.5). Amplitude of the simulated component is about $0.01 \mu \mathrm{V}$ to $0.02 \mu \mathrm{V}$, a little less than the $\mathrm{V}_{\mathrm{pp}}$ of a single SFAP and lower than the animal experimental result. The width of the simulated component is around $1 \mathrm{~ms}$, whereas the animal experimental result shows a wider component. Also in contrast to the animal experimental results, the simulated component was observed on all three electrodes. The modelled dispersed component can be made to resemble the animal experimental result more in amplitude by increasing the number of SFAPs (figure 4.6). The width of the component can be increased by adding SFAPs of thicker and thinner fibres, since these are recorded before and after the original response respectively (figure 4.6). When the SFAP timing was randomized (figure 4.4), the distinct shape disappeared and a noisy signal was recorded (figure 4.4). Standard deviation of this noisy signal was about $0.04 \mu \mathrm{V}$, which is a little higher than once the SFAP $V_{p p}$.

The modelled dispersed component is influenced by varying $\tau$, the parameter that shapes the bell-shaped pattern of arrival time $\lambda$. When $\tau$ is decreased, the bell-shaped pattern of $\lambda$, as shown in figure 4.4, becomes narrower (figure 4.7). For values of $\tau$ between $1 \mathrm{~ms}$ and $2 \mathrm{~ms}$, the resulting component is a summation of almost simultaneous SFAPs, giving the width of a SFAP (around $0.4 \mathrm{~ms}$, figure 4.8), and an amplitude of a summation of 500 SFAPs (around $15 \mu \mathrm{V}$, figure 4.8). When the random condition is used for the calculation of $\lambda$, an increase in $\tau$ above $2 \mathrm{~ms}$ leads to a signal similar to spontaneous neural activity with a slight increase of activity in the middle of the bell shape (as in figure 4.4). There is no longer a component to determine width and amplitude from. In the non-random condition, when $\tau$ is increased above $2 \mathrm{~ms}$ the SFAPs add up to a dispersed component (as in figure 4.4) with a larger width than the SFAP and a smaller but non-zero amplitude. For all parameter settings, a dispersed component was found either with all electrodes in the average reference method, or with none of the electrodes. 


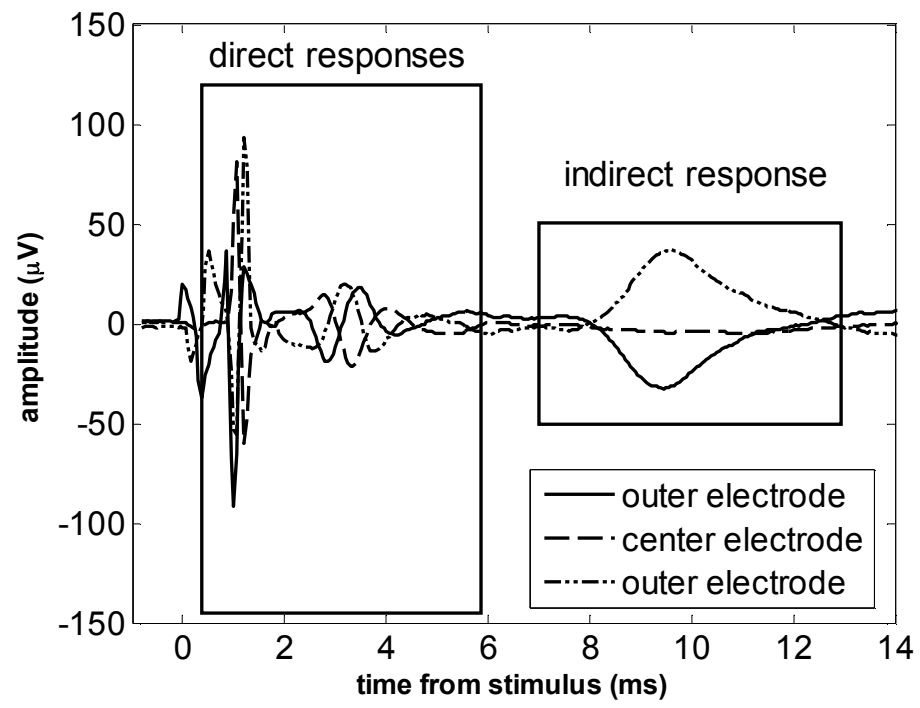

Figure 4.5: Example of an animal experimental result obtained from a pig of an indirect response in the evoked compound action potential. The component was more pronounced on an outer ring electrode, than on the centre ring electrode using the average reference configuration.

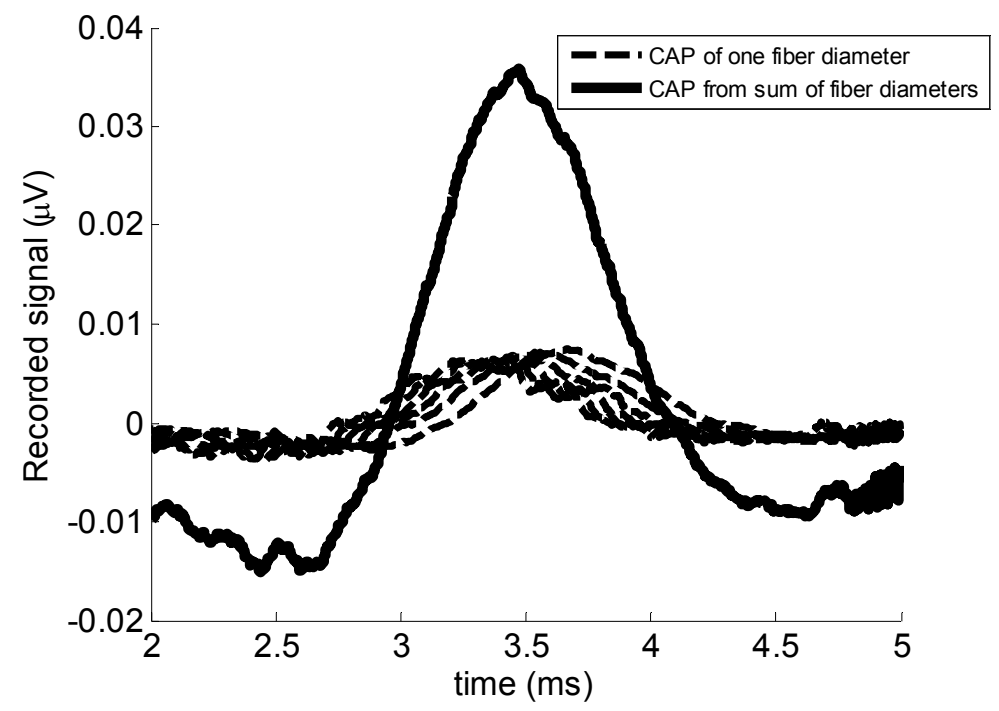

Figure 4.6: A summation of indirect components as presented in figure 4.4 (nonrandom condition) for fibres of $7 \mu \mathrm{m}, 8 \mu \mathrm{m}, 9 \mu \mathrm{m}, 10 \mu \mathrm{m}, 11 \mu \mathrm{m}$, and $12 \mu \mathrm{m}$. The dashed lines represent the components for a single diameter. The component's amplitude increases because the number of SFAPs is increased. The component's width increases because the range of fibre diameters is increased, because the smaller diameter fibres arrive later in the cuff. 


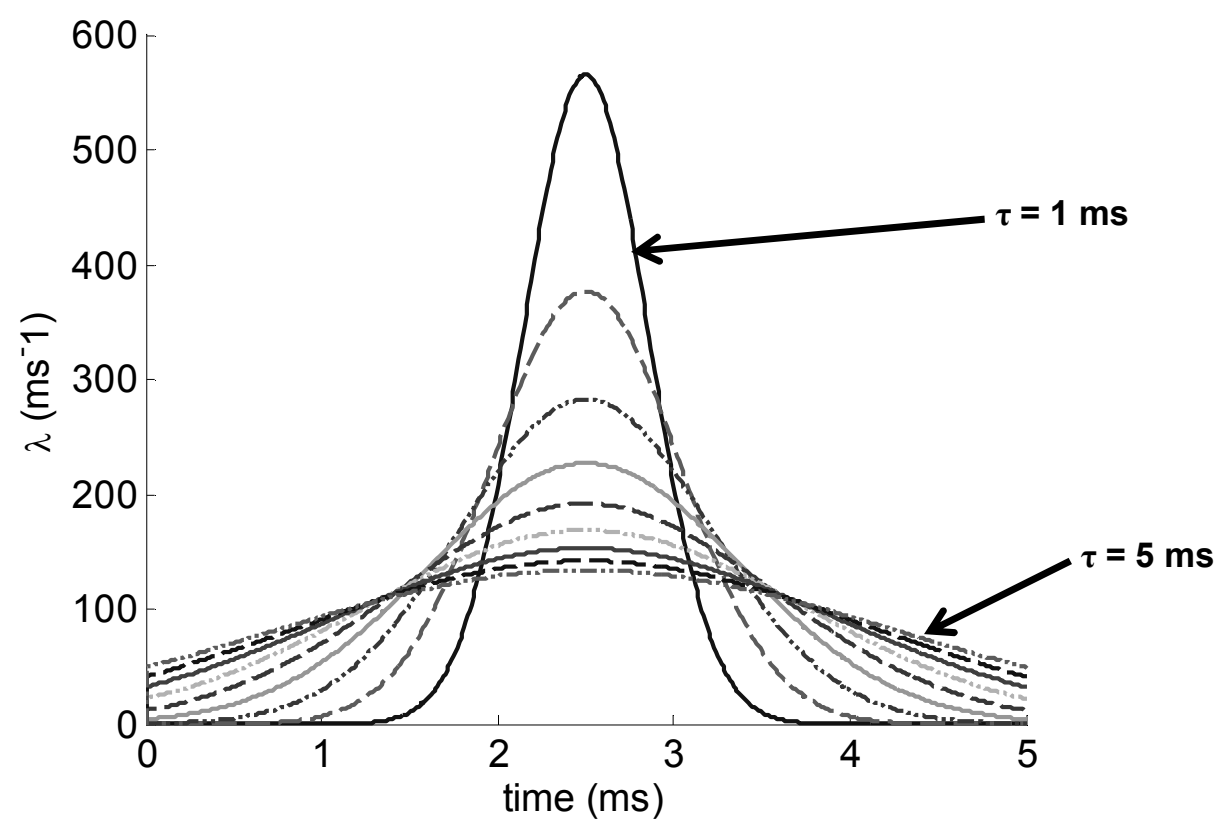

Figure 4.7: The effect of $\tau$ on the $\lambda$ pattern during the burst of SFAPs that create the indirect component. Total number of SFAPs and length of the time window are kept constant. 

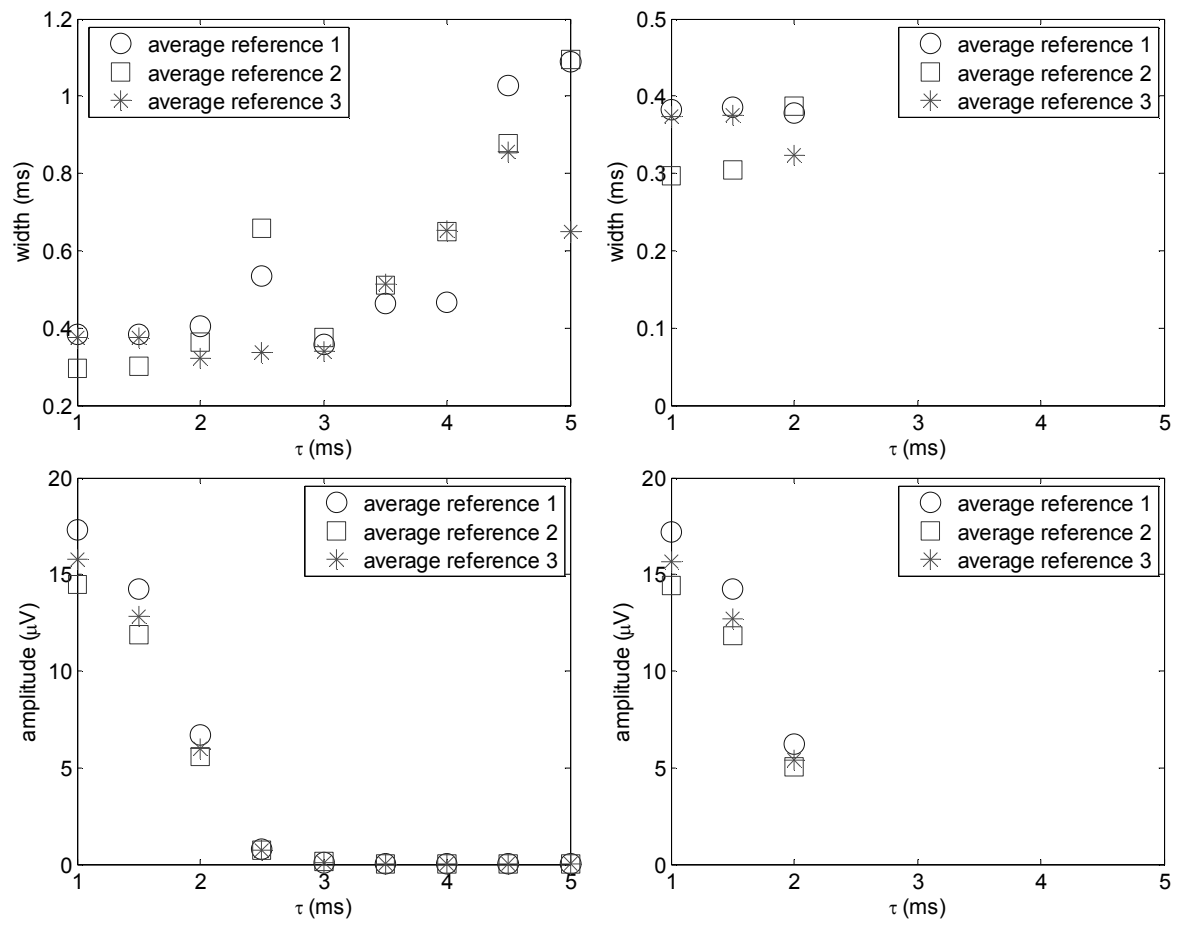

Figure 4.8: Effect of $\tau$ (as in equation (4.13)) on the width (top panels) and amplitude (bottom panels) of the component when $\lambda$ is determined in the random condition (right panels) or non-random condition (left panels). For values of $\tau$ between $1 \mathrm{~ms}$ and $2 \mathrm{~ms}$, the resulting component is a summation of almost simultaneous SFAPs, giving the width SFAP (between 0.3 and $0.4 \mathrm{~ms}$ ) and the amplitude of a summation of 500 SFAPs (about $15 \mu \mathrm{V}$ ). For the random condition (right panels), when $\tau$ increases further the shape of the SFAPs can no longer be discerned and a signal similar to spontaneous neural activity results. Therefore, there is no longer a component to determine width and amplitude from. In the non-random condition, the SFAPs add up to a dispersed component with a larger width than the SFAP (top left panel) and a smaller but nonzero amplitude (top right panel). 


\subsection{Discussion}

A burst of SFAPs with a bell shaped pattern of arrival times $\lambda$ resulted in a dispersed component. If timing of subsequent SFAPs is randomized, a noisy signal results and no clear component is observed. This means that cancellation of the SFAP signals occurs when the random condition is used. If the bell shape of the $\lambda$ pattern is made smaller, dispersion is reduced and only the SFAP shape remains, as is the case for regular CAP recording. This shows that there is only a small range of burst characteristics that leads to a dispersed component. The amplitude and width of the modelled component are smaller than in the animal experimental results. The amplitude of the indirect component increases when the number of SFAPs is increased. The width of the component can be increased by adding SFAPs that have a larger diameter range or enter the cuff over a larger range of arrival times. The maximum amplitude and width of the component is limited by the number of SFAPs in the model, since it is assumed that each fibre creates a SFAP only once in the considered time window and can therefore be used only once to add width when timed correctly. A number of 500 SFAPs was used, since this approximates the number of large myelinated fibres in the vagus nerve and it was assumed that each fibre fires only once in the evoked component. The difference between the modelled component and the animal experimental results may indicate that more fibres than the 500 assumed contribute to the indirect component, that a larger population of fibre diameters contributes to the component, or that each fibre fires multiple times after one electrical stimulus. This may be the case when the response occurs via a neuronal network.

A dispersed component was recorded in the non-random condition, but not in the random condition. In the random condition a narrow non-dispersed component could be modelled only with small values of $\tau$. For larger values of $\tau$ the resulting signal resembled spontaneous neural activity. It is unlikely that the arrival times of SFAPs in the cuff are completely non-random and follow the pattern of a bell-shape exactly when they originate from the neural network on the heart, which may result in various latencies. However, if the component is evoked after an electrical stimulus, the response may be synchronized because of the electrical pulse and the arrival times may be less randomized than. Therefore, it is feasible that the modelled dispersed component as presented in this chapter resembles a physiological process.

The model shows that in case the component is the result of a burst of SFAPs with varying latencies, the centre electrode recorded the dispersed component with a similar amplitude as the outer electrodes. A notable difference between the animal experiments and our computer model is that in the animal experiments the dispersed component was strongly reduced on the centre electrode. This could indicate that the parameter values used in our model to 
reproduce the dispersed component did not match the physiological situation, or that the hypothesized physiological origin, i.e. a burst of SFAPs with varying latencies, is not correct.

Potentially the indirect component is not a result of evoked nerve activity, but originates from nearby muscular activity (e.g. from the larynx). Interference from outside the cuff, such as from active muscles, can be reduced by a tripolar derivation [12-14]. This is because the potential field inside a cuff generated by a source outside the cuff changes linearly with position, which is rejected by a tripolar derivation [15]. The weight function of a tripolar derivation (equation 4.8) shows that the interference will be zero. The interference will also be zero on the centre electrode in the average reference configuration, since its derivation is identical except for a factor of $2 / 3$ (equation 4.9). This could explain why in the animal experiment the indirect component was reduced on the centre electrode compared to the outer electrodes using the average reference configuration.

\subsection{Conclusions}

The occurrence of a dispersed, fast neural component can be explained by the hypothesized physiological origin of a dispersed burst of fast SFAPs of nonrandom nature. The fact that the simulated dispersed component was similar on the centre electrode and the outer electrodes does not support this hypothesis.

\section{Appendix}

Equations and parameters to model action potential conduction, as described in $[6]$.

\section{Equations}

$$
\begin{aligned}
& \mathrm{a}_{\mathrm{m}}=4.6 \times 10^{3}(\mathrm{~V}+18.4) /\left(1-\mathrm{e}^{(-18.4-\mathrm{V}) / 10.3}\right) \\
& \mathrm{b}_{\mathrm{m}}=0.33 \times 10^{3}(-22.7-\mathrm{V}) /\left(1-\mathrm{e}^{(\mathrm{V}+22.7) / 9.16}\right) \\
& \mathrm{a}_{\mathrm{h}}=0.21 \times 10^{3}(-111-\mathrm{V}) /\left(1-\mathrm{e}^{(\mathrm{V}+111) / 11}\right) \\
& \mathrm{b}_{\mathrm{h}}=14.1 \times 10^{3} /\left(1+\mathrm{e}^{(-28.8-\mathrm{V}) / 13.4}\right) \\
& \mathrm{a}_{\mathrm{n}}=51.7(\mathrm{~V}+93.2) /\left(1-\mathrm{e}^{(-93.2-\mathrm{V}) / 1.1}\right) \\
& \mathrm{b}_{\mathrm{n}}=92(-76-\mathrm{V}) /\left(1-\mathrm{e}^{(\mathrm{V}+76) / 10.5}\right) \\
& \mathrm{dm} / \mathrm{dt}=\mathrm{a}_{\mathrm{m}}(1-\mathrm{m})-\mathrm{b}_{\mathrm{m}} \mathrm{m} \\
& \mathrm{dh} / \mathrm{dt}=\mathrm{a}_{\mathrm{h}}(1-\mathrm{h})-\mathrm{b}_{\mathrm{h}} \mathrm{h} \\
& \mathrm{dn} / \mathrm{dt}=\mathrm{a}_{\mathrm{n}}(1-\mathrm{n})-\mathrm{b}_{\mathrm{n}} \mathrm{n}
\end{aligned}
$$


$\mathrm{i}_{\mathrm{Na}}=\mathrm{m}^{3} \mathrm{hp}_{\mathrm{Na}} \mathrm{VF}^{2}\left(\mathrm{Na}_{\mathrm{o}}-\mathrm{Na}_{\mathrm{i}} \mathrm{e}^{\mathrm{VF} / \mathrm{RT}}\right) / \mathrm{RT}\left(1-\mathrm{e}^{\mathrm{VF} / \mathrm{RT}}\right)$

$\mathrm{i}_{\mathrm{K}}=\mathrm{n}^{4} \mathrm{~g}_{\mathrm{K}}\left(\mathrm{V}-\mathrm{V}_{\mathrm{K}}\right)$

$\mathrm{i}_{\mathrm{L}}=\mathrm{g}_{\mathrm{L}}\left(\mathrm{V}-\mathrm{V}_{\mathrm{L}}\right)$

$\mathrm{i}_{\text {ion }}=\mathrm{i}_{\mathrm{Na}}+\mathrm{i}_{\mathrm{K}}+\mathrm{i}_{\mathrm{L}}$

$\mathrm{i}_{\mathrm{c}}=\mathrm{c}_{\mathrm{m}} \mathrm{dV} / \mathrm{dt}$

$\mathrm{I}_{\text {mem }}=\left(\mathrm{i}_{\text {ion }}+\mathrm{i}_{\mathrm{c}}\right) \pi \mathrm{dl}$

$\mathrm{m}(0)=0.0382$

$\mathrm{h}(0)=0.6986$

$\mathrm{n}(0)=0.2563$

\section{Parameters}

$\mathrm{D}=$ fibre diameter $[\mathrm{m}]$

$\mathrm{L}=$ internodal length $[\mathrm{m}]$

$1=$ nodal length $[\mathrm{m}]$

$\mathrm{d}=$ axon diameter [m], estimated as $0.6^{*} \mathrm{D}[8]$

$\mathrm{c}_{\mathrm{m}}=$ membrane capacity, $0.028 \mathrm{~F} / \mathrm{m}^{2}$

$\mathrm{g}_{\mathrm{L}}=$ leakage conductance, $600 \mathrm{~S} / \mathrm{m}^{2}$

$\mathrm{p}_{\mathrm{Na}}=$ sodium permeability, $0.0704 \mathrm{dm}^{3} / \mathrm{m}^{2} \mathrm{~s}$

$\mathrm{g}_{\mathrm{K}}=$ potassium conductance, $300 \mathrm{~S} / \mathrm{m}^{2}$

$\rho_{\mathrm{a}}=$ intra-axonal resistance, $0.33 \Omega \mathrm{m}$

$\mathrm{V}_{\mathrm{L}}=$ leakage equilibrium potential, $-84.14 \mathrm{mV}$

$\mathrm{V}_{\mathrm{Na}}=$ sodium equilibrium potential, $43.7 \mathrm{mV}$

$\mathrm{Na}_{\mathrm{o}}=$ sodium concentration outside cell, $154 \mathrm{mM}$

$\mathrm{Na}_{\mathrm{i}}=$ sodium concentration inside cell, $30 \mathrm{mM}$

$\mathrm{V}_{\mathrm{K}}=$ potassium equilibrium potential, $-84 \mathrm{mV}$

$\mathrm{F}=$ Faraday constant, $96485 \mathrm{C} / \mathrm{mole}$

$\mathrm{R}=$ gas constant, $8.3144 \mathrm{~J} / \mathrm{K}$ mole

$\mathrm{T}=$ absolute temperature, $310.15 \mathrm{~K}$

$\mathrm{i}_{\mathrm{Na}}=$ sodium current $\left[\mathrm{A} / \mathrm{m}^{2}\right]$

$\mathrm{i}_{\mathrm{K}}=$ potassium current $\left[\mathrm{A} / \mathrm{m}^{2}\right]$

$\mathrm{i}_{\mathrm{L}}=$ leakage current $\left[\mathrm{A} / \mathrm{m}^{2}\right]$

$\mathrm{i}_{\text {ion }}=$ total ionic current $\left[\mathrm{A} / \mathrm{m}^{2}\right]$

$\mathrm{i}_{\mathrm{c}}=$ capacitive current $\left[\mathrm{A} / \mathrm{m}^{2}\right]$

$\mathrm{I}_{\mathrm{mem}}=$ total nodal membrane current $[\mathrm{A}]$

\section{References}

1. Ordelman, S.C.M.A., L. Kornet, R. Cornelussen, H.P. Buschman, and P.H. Veltink (2010) An indirect component in the evoked compound action potential of the vagal nerve J Neural Eng, 7(6), p. 066001. 
2. Hursh, J.B. (1939) Conduction velocity and diameter of nerve fibres Am J Physiol, 127, p. 131-9.

3. Agostoni, E., J.E. Chinnock, M.B. De Daly, and J.G. Murray (1957) Functional and histological studies of the vagus nerve and its branches to the heart, lungs and abdominal viscera in the cat J Physiol, 135(1), p. 182-205.

4. Offner, F.F. (1950) The EEG as potential mapping - the value of the average monopolar reference Electroen Clin Neuro, 2(2), p. 213-4.

5. Taylor, J., N. Donaldson, and J. Winter (2004) Multiple-electrode nerve cuffs for low-velocity and velocity-selective neural recording Med Biol Eng Comput, 42(5), p. 634-43.

6. Wesselink, W.A., J. Holsheimer, and H.B. Boom (1999) A model of the electrical behaviour of myelinated sensory nerve fibres based on human data Med Biol Eng Comput, 37(2), p. 228-35.

7. Andreasen, L.N.S., J.J. Struijk, and S. Lawrence (2000) Measurement of the performance of nerve cuff electrodes for recording Med Biol Eng Comput, 38(4), p. 447-53.

8. Struijk, J.J. (1997) The extracellular potential of a myelinated nerve fibre in an unbounded medium and in nerve cuff models Biophysical Journal, 72(6), p. 2457-69.

9. Veltink, P.H., T. Tonis, H.P.J. Buschman, E. Marani, and W.A. Wesselink. Multi-electrode nerve cuff recording - model analysis of the effects of finite cuff length. in 3rd European Medical and Biological Engineering Conference EMBEC'05. 2005. Prague, Czech Republic: Springer Verlag.

10. Andreasen, L.N. and J.J. Struijk (2002) Signal strength versus cuff length in nerve cuff electrode recordings IEEE Trans Biomed Eng, 49(9), p. 1045-50.

11. Crowley, T.H. (1954) On reciprocity theorems in electromagnetic theory Journal of Applied Physics 25(1), p. 119-20.

12. Rahal, M., J. Taylor, and N. Donaldson (2000) The effect of nerve cuff geometry on interference reduction: A study by computer modeling IEEE Trans Biomed Eng, 47(1), p. 136-8.

13. Rahal, M., J. Winter, J. Taylor, and N. Donaldson (2000) An improved configuration for the reduction of emg in electrode cuff recordings: A theoretical approach IEEE Trans Biomed Eng, 47(9), p. 1281-4.

14. Stein, R.B., D. Charles, L. Davis, J. Jhamandas, A. Mannard, and T.R. Nichols (1975) Principles underlying new methods for chronic neural recording Can J Neurol Sci, 2(3), p. 235-44.

15. Struijk, J.J. and M. Thomsen. Tripolar nerve cuff recording: Stimulus artifacts, emg, and the recorded nerve signal. in IEEE/EMBS 17th Ann Int Conf. 1995. 


\title{
Chapter 5
}

\section{Additional experimental data \\ on the origin of the late component in the evoked vagus nerve compound action potential}

\author{
Simone Ordelman \\ Lilian Kornet \\ Richard Cornelussen \\ Enrico Marani \\ Peter Veltink
} Submitted 
This chapter concerns additional insights related to our hypothesis on the physiological origin of an additional component found in the evoked compound action potential (CAP) of the vagus nerve, as published previously [1]. We hypothesized that this component emanates from the heart neuronal network. Additional experiments indicate that the larynx is involved.

An additional component was found in the evoked CAP that had a high velocity along the nerve (about $70 \mathrm{~m} / \mathrm{s}$ ) but a long latency, from which we concluded that this was an indirect neuronal component. The component's direction along the nerve showed that it came from the periphery, suggesting an afferent pathway. This additional component was recorded on the left and right vagus nerve simultaneously after unilateral stimulation, which possibly indicates a bilateral reflexive pathway. The shape was dispersed and the component was strongly reduced when a tripolar derivation was taken. The tripolar derivation takes a second order spatial derivative of a dispersed signal. A dispersed shape that is wider than the cuff will have a close to linear field in the cuff and will be strongly reduced with such a derivation. This also explains why this signal was not reported in the literature before.

Important arguments that were used in [1] that supported the hypothesis of the component being of cardiovascular origin are: the effects of heart rate, blood pressure and contractility on the component's latency. Dobutamine was administered to increase heart rate, blood pressure and contractility and it decreased the component's latency. In unpublished experiments, burst vagal nerve stimulation $(50 \mathrm{~Hz}, 1-10 \mathrm{~mA})$ was used to reduce heart rate, blood pressure and contractility which led to a simultaneously increased latency of the component. It was hypothesized that changes in the signals generated by the heart neuronal network caused these latency effects.

To further elucidate the physiological origin of this component, additional experiments were performed. In this chapter we present the results of two experiments in which the influence of the larynx on the recorded signal was examined. In two pigs, the left cervical vagus nerve was electrically stimulated at $1 \mathrm{~Hz}$ and recorded from using cuff electrodes as described previously [1]. On the left side, the superior laryngeal nerve, the recurrent laryngeal nerve and the vagus nerve caudal to the electrodes were cut consecutively, while vagus nerve recording and electrical stimulation was continued at a level that elicited a CAP after each stimulus.

In both pigs, the CAP was recorded including the additional component. Cutting the superior laryngeal nerve did not affect the number of components or the amplitude of the CAP. After cutting the recurrent laryngeal nerve the additional component had disappeared in both pigs. Cutting the vagus nerve after the superior and recurrent laryngeal nerves were cut did not further influence the 
CAP. These results show involvement of the recurrent laryngeal nerve in the additional component of the CAP.

We present various arguments in favour of a muscular or neuronal origin. An argument for a neuronal origin of the CAP component is that it has a velocity of approximately $40 \mathrm{~m} / \mathrm{s}$ along the nerve, which was estimated from relative delays of the component both within the cuff and between two cuffs [1]. This is not reflective of conduction along the muscle, since typical muscle conduction velocity is in the range of 2 to $5 \mathrm{~m} / \mathrm{s}$ [2]. Alternatively, if the component would be of muscular origin the activity may be recorded almost simultaneously on all electrodes. However, an apparent fast conduction velocity, characteristic of thicker myelinated fibres may result from superposition of a relatively slow B or $\mathrm{C}$ nerve fibre response on the muscular signal. Such a slow response remained after cutting the recurrent laryngeal nerve.

Another argument is the CAP component's latency of about $8 \mathrm{~ms}$, which was used as an indication of the distance from which the component had originated, leading to the heart neuronal network as a feasible source [1]. However, the latency also matches the time it takes for a laryngeal muscular contraction to occur after cervical vagus stimulation [3].The reduced amplitude with a tripolar derivation supports the hypothesis that the component is of muscular origin, since tripolar derivations are known to reduce muscular interference [4-6].

Moreover, the observed change in the component's latency with changes in heart rate, blood pressure and contractility may or may not have a causal relation. The effect on the heart may influence the recorded component at the point where the recurrent laryngeal nerve loops around the large arteries and aorta [7], either by neural connections or mechanical compression. However, the possibility of a muscular origin can also not be rejected, since dobutamine may have decreased the latency of muscular contraction, by increasing muscular contractility [8-9].The effects of burst stimulation on the latency could be due to muscle fatigue [10].

Finally, the dispersed shape of the CAP component may be the result of a summation of fast and narrow single fibre action potentials, with varying delays. However, the shape also resembles an evoked muscular response of the larynx, which also has a dispersed shape and a width of a few milliseconds [3]. The component was observed on both the left and right vagus nerve approximately simultaneously when stimulating on one side, which could be explained by a bilateral neural reflexive loop via the heart neuronal network. A bilateral neural pathway also exists through the recurrent laryngeal nerves, via the neuronal plexus at the level of the trachea [11]. The recorded component possibly travelled via this pathway. The bilateral component may also be explained as muscular activity from the larynx, since this would be conducted 
simultaneously trough the tissue on both sides. An evoked muscular response can result from stimulation on either the left or right vagus nerve.

At this point, it is certain that the pathway of the additional CAP component as reported in [1] involves the recurrent laryngeal nerve. However, it remains unclear whether it is of neuronal or muscular origin.

\section{Acknowledgement}

We would like to thank the reviewer of our unpublished experiments for his or her suggestion to investigate the role of the larynx.

\section{References}

1. Ordelman, S.C.M.A., L. Kornet, R. Cornelussen, H.P. Buschman, and P.H. Veltink (2010) An indirect component in the evoked compound action potential of the vagal nerve J Neural Eng, 7(6), p. 066001.

2. Andreassen, S. and L. Arendt-Nielsen (1987) Muscle fibre conduction velocity in motor units of the human anterior tibial muscle: A new size principle parameter J Physiol, 391, p. 561-71.

3. Castoro, M.A., P.B. Yoo, J.G. Hincapie, J.J. Hamann, S.B. Ruble, P.D. Wolf, and W.M. Grill (2011) Excitation properties of the right cervical vagus nerve in adult dogs Exp Neurol, 227(1), p. 62-8.

4. Rahal, M., J. Taylor, and N. Donaldson (2000) The effect of nerve cuff geometry on interference reduction: A study by computer modeling IEEE Trans Biomed Eng, 47(1), p. 136-8.

5. Rahal, M., J. Winter, J. Taylor, and N. Donaldson (2000) An improved configuration for the reduction of emg in electrode cuff recordings: $A$ theoretical approach IEEE Trans Biomed Eng, 47(9), p. 1281-4.

6. Stein, R.B., D. Charles, L. Davis, J. Jhamandas, A. Mannard, and T.R. Nichols (1975) Principles underlying new methods for chronic neural recording Can J Neurol Sci, 2(3), p. 235-44.

7. Gray, H., R. Warwick, P. Williams, M. Dyson, L. Bannister, and R. Moore, Gray's anatomy. 1980: Churchill Livingstone.

8. Ebata, T., Y. Fujii, and H. Toyooka (1992) Dobutamine increases diaphragmatic contractility in dogs Can J Anaesth, 39(4), p. 375-80.

9. Holmberg, E. and B. Waldeck (1977) Analysis of the beta-receptor mediated effect on fast-contracting skeletal muscle in vitro Naunyn Schmiedebergs Arch Pharmacol, 301(2), p. 109-13.

10. Bigland-Ritchie, B., D.A. Jones, and J.J. Woods (1979) Excitation frequency and muscle fatigue: Electrical responses during human voluntary and stimulated contractions Exp Neurol, 64(2), p. 414-27.

11. Rusu, M.C., F. Pop, A.L. Boscu, A.M. Jianu, D. Dermengiu, G.C. Curca, and S. Hostiuc (2011) Anatomical and immunohistochemical considerations on the microinnervation of trachea in humans Ann Anat, 193(1), p. 13-22. 


\title{
Chapter 6
}

Evoked responses from cranial origin in the cervical vagus

nerve

\author{
Simone Ordelman \\ Lilian Kornet \\ Richard Cornelussen \\ Rik Buschman \\ Peter Veltink
}




\section{Abstract}

The vagus nerve is involved in reflexive control of the cardiovascular system. However, in the evoked compound action potential of the vagus nerve, no reflexive components coming from the brain stem have been observed. In this chapter, wire electrodes and recordings in animals that are awake were used in an attempt to find such reflexive components. In one out of three pigs a component from the direction of the brain was found with wire electrodes under anaesthesia. In one out of five pigs such a component was found when the pig was awake. These results suggest that reflexive components coming from the brain are present in the evoked compound action potential, but are challenging to record. 


\subsection{Introduction}

In this chapter, evoked vagal responses are studied for their possible link to cardiovascular control. The response of fibres in the vagus nerve, evoked by electrical stimulation, has been studied in both animals and humans [1-3]. These compound action potentials (CAPs) consist of components, which emanate from thick, myelinated fibres to thin, unmyelinated fibres. The vagus nerve is involved in reflexive control of the heart; therefore the evoked CAP potentially contains reflexive components of cardiac fibres. Such components may be relevant as input to a closed-loop vagal stimulator for cardiovascular disease. In our previous research, we hypothesized that stimulating the afferent limb of the baroreceptor reflex would results in a reflexive component in the CAP from the efferent limb of the baroreceptor reflex. However, reflexive components coming from the brain stem were not found [4]. One possible explanation for this result is that the signals from the efferent limb of the baroreceptor reflex have an amplitude too low to record with our method of whole nerve recording, since they are of the small diameter myelinated kind [5]. To overcome this, in the current study we attempted to record small amplitude signals with a wire electrode inserted into the nerve. Alternatively, the reflexive components may have been suppressed by anaesthetics [6]. We recorded evoked CAPs in pigs that were awake to address this possibility.

\subsection{Methods}

The experiments were performed in eight female Dutch Landrace pigs. The pig was premedicated with $0.5 \mathrm{mg} / \mathrm{kg}$ Morphine 20 minutes before induction with $3 \mathrm{mg} / \mathrm{kg}$ Zoletil (Telazol) and $0.5 \mathrm{mg} / \mathrm{kg}$ xylazine. The pig was anesthetized with a combination of isoflurane $(1-1.5 \%)$ and fentanyl $(6-10 \mu \mathrm{g} / \mathrm{kg} / \mathrm{hr})$. The left vagus nerve was dissected free from surrounding tissue at the cervical level. Two cuff electrodes with three circular electrode sites were used for electrical stimulation and recording respectively, with the recording cuff cranial to the stimulation cuff (figure 6.1a). Electrical nerve stimulation was performed with a custom-made stimulator, with a train of 30 individual pulses $(1 \mathrm{~Hz}, 300 \mu \mathrm{s}, 0.1$ $5 \mathrm{~mA}$ ). Neural recordings were amplified with a TMSi Refa amplifier (TMS international, Oldenzaal, The Netherlands). The data were sampled at $20 \mathrm{kHz}$ and stored on a laptop using PortiLab2 software (TMS international, Oldenzaal, The Netherlands). In three pigs, three wire electrodes were inserted into the vagus nerve cranially to the cuff electrodes to record neural signals (figure 6.1b). After a stimulus train was delivered to the nerve, the nerve was cut cranially to the electrodes and another stimulus train was delivered. Next, the nerve was cut caudally to the electrodes and another stimulus train was delivered to the nerve.

The other five pigs were awoken after implantation of the cuff electrodes. The next day, while the pig was awake, recordings of CAPs were made. 


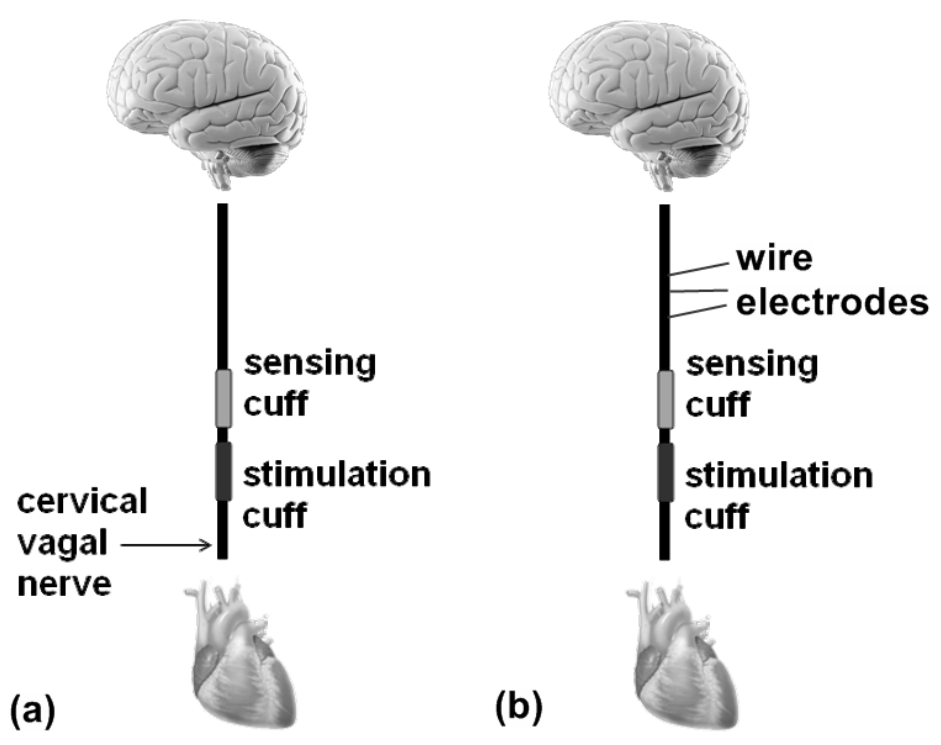

Figure 6.1: Schematic representation of the experimental setup with two cuff electrodes (a), and with the addition of wire electrodes (b).

The signals were analyzed offline in Matlab R2007a (The Mathworks Inc.). Using the stimulation artefacts as reference points, CAPs from the same stimulation train were ensemble averaged. A component in the CAP was identified as such, if it occurred after each stimulus before ensemble averaging and had an amplitude after ensemble averaging of at least two times the standard deviation of the signal before the stimulus. Latency of each component of the CAP was determined as the time between the stimulus artefact and the highest initial slope of a component. Velocity and direction along the nerve were derived from CAPs recorded at consecutive electrode sites (chapter three, [4]).

\subsection{Results}

Using wire electrodes, in one out of three pigs a component of the CAP was found that was not observed in our previous study with cuff electrodes (figure 6.2). It had a latency of about $37 \mathrm{~ms}$. It disappeared when the nerve was cut cranially to the electrodes. Direction and velocity could not be determined from this recording, because only one wire recorded the component. The component was observed at a stimulation amplitude of $5 \mathrm{~mA}$. The exact threshold could not be determined, because in this pig a signal was only recorded in the recording with a stimulation amplitude of $5 \mathrm{~mA}$. 


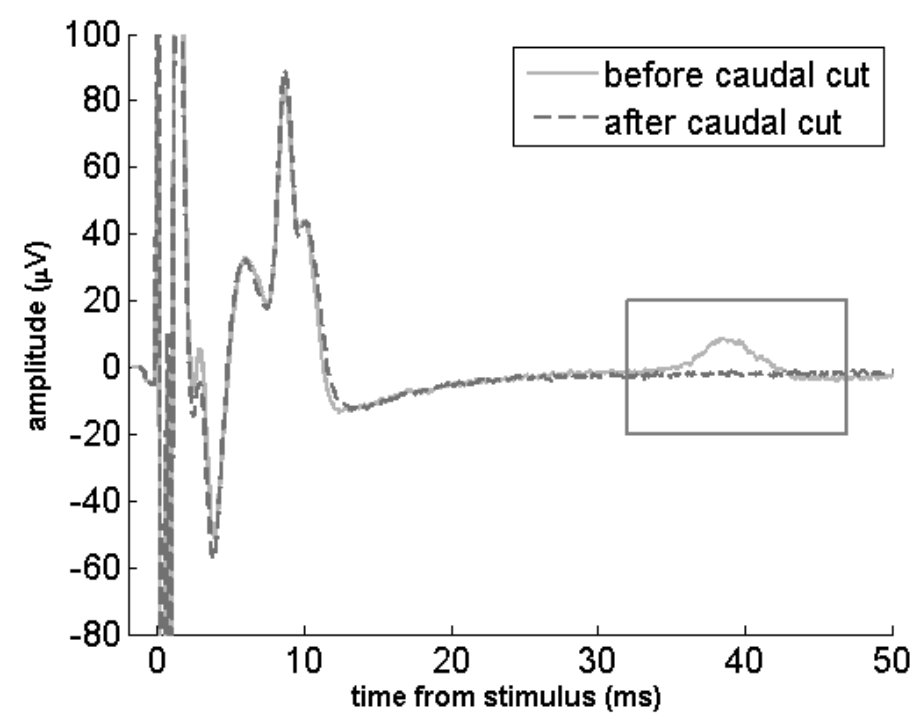

Figure 6.2: Recording with wire electrodes. The box indicates a component that disappeared when the nerve was cut caudal to the electrodes.

Additional components to that found during the anesthetized condition were found in three out of the five pigs when the pig was awake. One additional component was found in two pigs, at $20 \mathrm{~ms}$ and $23 \mathrm{~ms}$ respectively. In the third pig, two additional components were found at $15 \mathrm{~ms}$ and $27 \mathrm{~ms}$ respectively (figure 6.3). Only the component with a latency of $27 \mathrm{~ms}$ in the third pig came from the direction of the brain. This component had a velocity of $7 \mathrm{~m} / \mathrm{s}$ and a threshold between $0.1 \mathrm{~mA}$ and $1 \mathrm{~mA}$. The other additional components had a low velocity between $2 \mathrm{~m} / \mathrm{s}$ and $6 \mathrm{~m} / \mathrm{s}$. The threshold for these components was below $1.5 \mathrm{~mA}$. 

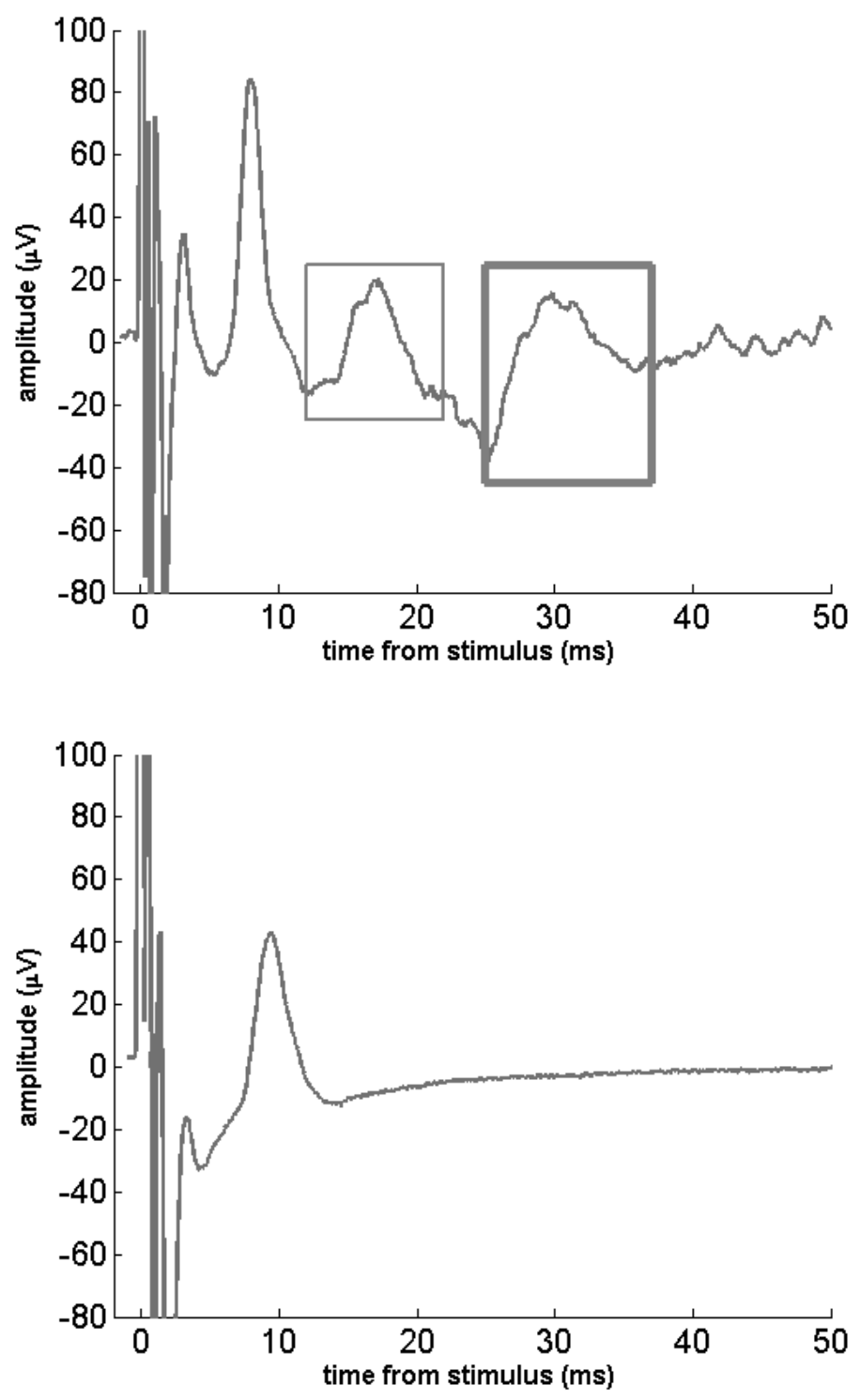

Figure 6.3: Recording in a pig when anesthetized (top) compared to a recording when the pig was awake (bottom). In this pig, two components were found that were not present in the anesthetized pigs. The boxes indicate these components. The component with the thick box came from the direction of the brain. 


\subsection{Discussion}

In only one out of three pigs where a wire electrode was used, a component was found that was not observed with cuff electrodes in anesthetized recordings. This component disappeared when the nerve was cut cranially to the electrodes, showing that this component came from the direction of the brain. It is likely part of a reflexive loop from the brain stem, which was interrupted when the nerve was cut. It was only observed when inserting a wire electrode, possibly because it originates from small diameter fibres which generate small amplitude signals. This signal may be obscured by larger signals when a whole nerve recording is made with a cuff electrode. Threshold and velocity could not be analyzed in this recording, making it unclear what fibre type originated this response. The lack of additional components in the other two pigs may be explained by the difference in wire positioning in the nerve and variations in fibre distribution.

In recordings made in the pigs that were awake, additional components to the anesthetized condition were found in three out of five pigs. It was concluded that these components were previously suppressed by anaesthetics. The additional components may have been from B-fibres, since their velocities of 2 $\mathrm{m} / \mathrm{s}$ to $7 \mathrm{~m} / \mathrm{s}$ are within the range of B-fibres [7]. The threshold for these components is below $1.5 \mathrm{~mA}$, which is low for the thin fibres that have the corresponding conduction velocity. In comparison, a component of type $\mathrm{B}$ fibres that was found in anesthetized pigs [4] had a velocity of $20 \pm 9 \mathrm{~m} / \mathrm{s}$ and a threshold of $2.17 \pm 1.27 \mathrm{~mA}$. A possible explanation is that a thicker fibre type was excited in an efferent path, which resulted in an afferent response of thin fibres. Not enough data is available at this point to test this hypothesis.

A component that came from the direction of the brain was found in only one out of three pigs that were awake. The velocity along the nerve of about $7 \mathrm{~m} / \mathrm{s}$ is within the range the small diameter myelinated efferent fibres of the baroreceptor reflex [8]. Fibres from aortic arch baroreceptors, which constitute the afferent limb of the baroreceptor reflex have a diameter of $4 \mu \mathrm{m}$ or $10 \mu \mathrm{m}$ [5]. This could explain the low stimulation threshold for the reflexive component in the CAP.

These results suggest that there is a reflexive component from the brain stem in the evoked CAP of the cervical vagus nerve. This has not been reported before. If this component is related to the baroreceptor reflex, it can provide clinically relevant information about the sensitivity of this reflex at the level of the brain stem. It is however difficult to record. Other electrode configurations should be tested for this purpose. For example the FINE cuff [9], this flattens the nerve within a cuff to reposition the fascicles with respect to the electrode contacts. Or a correctly positioned LIFE electrode [10], which is an intrafascicular electrode, may be able to record from the efferent cardiac fibres. With further research, the feasibility of recording reflexive components of the vagal CAP should be investigated. 


\section{References}

1. Tosato, M., Yoshida, K., Toft, E., Nekrasas, V., and Struijk, J.J. (2006) Closed-loop control of the heart rate by electrical stimulation of the vagus nerve Med Biol Eng Comput 44(3), p. 161-9

2. Evans, M.S., Verma-Ahuja, S., Naritoku, D.K., and Espinosa, J.A. (2004) Intraoperative human vagus nerve compound action potentials Acta Neurol Scand 110(4), p. 232-8

3. De Neef, K.J., Jansen, J.R.C., and Versprille, A. (1983) Attenuation of the negative chronotropic effect of myelinated fibres by nonmyelinated fibres in the vagus nerve of the rabbit Circ Res 52(2), p.508-14

4. Ordelman, S.C.M.A., L. Kornet, R. Cornelussen, H.P. Buschman, and P.H. Veltink (2010) An indirect component in the evoked compound action potential of the vagal nerve J Neural Eng, 7(6), p. 066001.

5. Agostoni, E., J.E. Chinnock, M.D.B. Daly, and J.G. Murray (1957) Functional and histological studies of the vagus nerve and its branches to the heart, lungs and abdominal viscera in the cat $\mathrm{J}$ Physiol-London, 135(1), p. 182-205.

6. Urban, B.W. (2002) Current assessment of targets and theories of anaesthesia Br J Anaesth, 89(1), p. 167-83.

7. Robinson, A.J. and L. Snyder-Mackler, Clinical electrophysiology : Electrotherapy and electrophysiologic testing. 3rd ed. 2008, Philadelphia: Wolters Kluwer/Lippincott Williams \& Wilkins. xv, p. 555

8. Kunze, D.L. (1972) Reflex discharge patterns of cardiac vagal efferent fibres J Physiol-London, 222(1), p. 1-15.

9. Tyler, D.J. and D.D.M. Durand (2002) Functionally selective peripheral nerve stimulation with a flat interface nerve electrode IEEE T Neur Sys Reh, 10(4), p. 294-303.

10. Zheng, X.J., M. Zhang, T.Y. Chen, and Z.W. Chen (2008) Recording and stimulating properties of chronically implanted longitudinal intrafascicular electrodes in peripheral fascicles in an animal model Microsurgery, 28(3), p. 203-9. 


\title{
Chapter 7
}

\section{The cardiac effects of vagus nerve stimulation}

\author{
Simone Ordelman \\ Lilian Kornet \\ Richard Cornelussen \\ Rik Buschman \\ Peter Veltink
}

Submitted 


\section{Abstract}

Electrical stimulation of the vagus nerve affects the cardiovascular system. When the nerves are left intact, reflexive loops can influence these effects.

Four cardiovascular parameters were analyzed: R-R interval, P-Q interval, left ventricular contractility, and left ventricular pressure. A first order exponential model was used to characterize each cardiovascular parameter's dynamics during vagus nerve stimulation. A time constant $\tau$ characterizes the exponential model for each parameter.

A return towards baseline values can occur for all four cardiovascular parameter while left vagus nerve stimulation is maintained. The dynamics of the initial cardiovascular effects of vagus nerve stimulation are significantly correlated between cardiovascular parameters. This also holds true when RRI is kept constant through atrial pacing. 


\subsection{Introduction}

When the cervical vagus nerve is stimulated with bursts of electrical stimuli, blood pressure and left ventricular contractility decrease, while R-R interval and $\mathrm{P}-\mathrm{Q}$ time increase [1-3]. It has been proposed that cardiac effects of vagus nerve stimulation (VNS) can be complex and inconsistent in the intact animal, due to reflexive mechanisms [4]. These dynamics are relevant for clinical applications of vagus nerve stimulation for cardiovascular effects.

Therefore, in this chapter the effects of left vagus nerve stimulation on heart rate (R-R interval), AV-node conduction time (P-Q interval), left ventricular contractility and left ventricular pressure have been analyzed with all neural pathways intact. Since we do not interfere with neural pathways, we expect our results to be influenced by sympathetic activation. This may lead to a return towards baseline of cardiovascular parameter values, while stimulation is continued, as reported in a case study for right vagus nerve stimulation [4]. We also expect to find a relation between the time dependencies of the effects on the four cardiovascular parameters, either through reflexive pathways (e.g. the baroreceptor reflex) or mechanical effects (e.g. a pressure-volume relationship).

To analyze both he initial effects of vagus nerve stimulation and the possible return towards baseline values during stimulation, we used a model to characterize the dynamics of the cardiovascular parameters during VNS. Several models have been proposed to describe the dynamics of the cardiovascular effects of VNS [5-7]. These models are of the first or second order, focus on one cardiovascular parameter and may include return towards baseline after stimulation. These models provide accurate descriptions of the cardiovascular effects of VNS, but most of these models are based on experiments in which the vagus nerves were cut or the sympathetic nervous system was blocked. We analyzed the parasympathetic (vagal) influence on the cardiovascular system by only including the initial effects observed during VNS, since it is known that the effects of sympathetic stimulation start with a delay [7]. Secondly, if a return towards baseline occurred while stimulation was maintained, this return towards baseline was analyzed separately.

\subsection{Methods}

\subsubsection{Experimental setup}

Experiments were performed in ten female Dutch Landrace pigs. Animal handling was performed according to the Dutch Law and the European Directive for the Protection of Animals and was approved by the Experimental Animal Committee of the Maastricht University. The pigs were premedicated with $0.5 \mathrm{mg} / \mathrm{kg}$ Morphine intramuscular (IM) 20 minutes before induction. Zoletil (Telazol), $3 \mathrm{mg} / \mathrm{kg}$, IM and xylazine $0.5 \mathrm{mg} / \mathrm{kg}$, IM were administered. 
Isoflurane was maintained once the pigs were intubated, the IV line was placed and the paws were shaved for fitting ECG electrodes. The pigs were anesthetized with a combination of isoflurane $(0-1.5 \%)$ and fentanyl (6-10 $\mu \mathrm{g} / \mathrm{kg} / \mathrm{hr}$ ). At the end of the experimental day, the pigs were euthanized with an overdose of pentobarbital.

The left vagus nerve was dissected free from the surrounding tissue at the cervical level and a custom-made self-coiling cuff electrode configuration was placed for electrical stimulation. The cuff was $15 \mathrm{~mm}$ long and had three circular Pt/Ir electrode contacts with an interelectrode distance of $4 \mathrm{~mm}$. The cuff was made in three diameters $(2,3.5$ and $5 \mathrm{~mm})$, to ensure a proper fit around the nerve. ECG recordings were made with surface electrodes. Left ventricular pressure was recorded with a Millar catheter (Millar Instruments, Houston, Texas, USA). ECG and blood pressure recordings were amplified with a TMSi Porti amplifier (TMS international, Oldenzaal, The Netherlands). ECG and $\mathrm{P}_{\mathrm{LV}}$ were sampled at $2048 \mathrm{~Hz}$. Data was stored on a laptop using PortiLab2 software (TMS international, Oldenzaal, The Netherlands).

The left vagus nerve was electrically stimulated using biphasic pulses generated by a custom-made stimulator. Pulse width was constant at $300 \mu \mathrm{s}$, pulse amplitude ranged from $1 \mathrm{~mA}$ to $10 \mathrm{~mA}$, and stimulation frequency ranged from $10 \mathrm{~Hz}$ to $50 \mathrm{~Hz}$. VNS was maintained for up to 60 seconds, unless unwanted side effects occurred such as complete heart block or digestive reflex.

The change in cardiovascular parameters was also assessed independently of heart rate. For this purpose, in three pigs, VNS was repeated during atrial pacing of the heart at a rate of at least ten percent above baseline levels, using the settings at which RR interval had increased most.

\subsubsection{Signal analysis}

For each pig, the recording with the maximum cardiovascular effect was selected, with a minimum of ten percent. In the selected recordings, a sinus rhythm was always maintained. Offline signal analysis was performed in Matlab R2007a (The Mathworks Inc.). P, Q and R-tops of the ECG were detected automatically and confirmed by visual inspection. R-R and P-Q intervals were determined. P-Q interval length (PQI) was normalized on heart cycle duration by dividing PQI by R-R interval length (RRI). Left ventricular contractility (LVC) was assessed using maximum values of the gradient of left ventricular pressure recordings: $\left(\frac{\mathrm{dP}_{\mathrm{LV}}}{\mathrm{dt}}\right)_{\max }$ [8]. Left ventricular systolic pressure $\left(\mathrm{P}_{\mathrm{LV}}\right)$ was determined using the values of the peaks of each cycle of the pressure recordings. Variations in cardiac parameters due to respiration were reduced by using non-overlapping four second averaging windows.

We used a first order model to describe the dynamics of parameters during burst stimulation. Before model fitting RRI, PQI, LVC and $\mathrm{P}_{\mathrm{LV}}$ were normalized with 
respect to their initial and peak value during burst stimulation. Outliers were removed. Linear interpolation was used to obtain a sampling frequency of $1 \mathrm{~Hz}$. The model was fitted to the section of the data which showed the initial rise or decline. Only the initial rise or decline was used for correlation analysis of the initial effects, to focus on the direct influence of the vagus nerve. The exponential first order was described by the parameter $\tau$ :

$y(t)=1-e^{-\frac{1}{\tau} t}$ to describe increasing parameters $y$, and

$y(t)=e^{-\frac{1}{\tau} t}$ to describe decreasing parameters $y$.

Time constant $\tau$ describes the rate of change. For a faster change, $\tau$ is smaller. The Levenberg-Marquardt method was used for nonlinear least-squares optimization. The goodness-of-fit of the model was evaluated by determining the coefficient of determination, which describes the variance accounted for:

$$
\mathrm{R}^{2}=1-\frac{\sum_{\mathrm{i}}\left(\mathrm{x}_{\mathrm{i}}-\mathrm{y}_{\mathrm{i}}\right)^{2}}{\sum_{\mathrm{i}}\left(\mathrm{x}_{\mathrm{i}}-\overline{\mathrm{x}}\right)^{2}}
$$

where $\mathrm{x}_{\mathrm{i}}$ is the normalized data, $\bar{x}$ is the mean value of the normalized data and $\mathrm{y}_{\mathrm{i}}$ is the fitted model. Only if $\mathrm{R}^{2}$ was 0.5 or higher the model was included in the analysis, because the model needs to follow the course of the data points. An exact fit to each data point is not necessary, since we are interested in changes with time and not in small fluctuations.

The effect of stimulation may reach a peak and then decrease again during stimulation. If this decrease was more than ten percent of the maximum effect, it was assumed to be brought about by reflexive processes, possibly from sympathetic origin. It was noted for each pig when such a return towards baseline occurred during VNS. The part of the signal that showed a return towards baseline was fitted with a first order model as described below, separately from the initial effect.

All parameters were tested for normality using a Shapiro-Wilk test. Normally distributed data were presented as mean \pm standard deviation, other data as median. Statistical significance of the effect of VNS on normally distributed parameters was tested with a one sample t-test. Effects on parameters that were not normally distributed were tested with a Wilcoxon test. Statistical significance of the results was tested at the $p<0.05$ level using the statistical analysis package SPSS.

Correlation analysis was used to assess the correlation between the dynamics of the cardiovascular parameters, by analyzing the correlation between their 
respective $\tau$ 's. Pearson's correlation coefficient $(r)$ is presented together with the $\mathrm{p}$-value to indicate if the slope was significantly different from zero.

For the three pigs in which the heart was paced initial effects on PQI, LVC and $\mathrm{P}_{\mathrm{LV}}$ were again fitted with a first order exponential model. If a return towards baseline of these parameters occurred it was again noted and fitted with a first order exponential model separately. The values of $\tau$ were compared between the paced and non-paced condition, but because of the small number of animals in the paced condition no statistical tests were performed.

\subsection{Results}

All four cardiac parameters changed significantly due to VNS. LVC decreased maximally by a median $9 \%(\mathrm{P}=0.005, \mathrm{n}=10)$. RRI increased maximally by a median $18 \%(\mathrm{P}=0.005, \mathrm{n}=10)$. $\mathrm{PQI}$ increased maximally by $36 \pm 40 \%$ $(\mathrm{P}=0.013, \mathrm{n}=10) . \mathrm{P}_{\mathrm{LV}}$ decreased maximally by $11 \pm 13 \%(\mathrm{P}=0.024, \mathrm{n}=10)$.

In two out of ten pigs a complete AV-block occurred during VNS with the highest amplitudes. In these cases VNS was repeated with an adjusted stimulation current to obtain a recording in which AV-conduction was maintained although possibly prolonged.

In all pigs the initial rise or decline of the cardiac parameters was observed and was fitted with the exponential first order model (Fig. 7.1). The value of $\mathrm{R}^{2}$ was lower than 0.5 in three pigs for the fit to PQI, in two pigs for the fit to LVC, and in one pig for the fit to $P_{\mathrm{LV}}$. These six fits were excluded from the analysis. This resulted in a total of ten $\tau_{\mathrm{RRI}}$ values, seven $\tau_{\mathrm{PQI}}$ values, eight $\tau_{\mathrm{LVC}}$ values, and nine $\tau_{\mathrm{PLV}}$ values. 

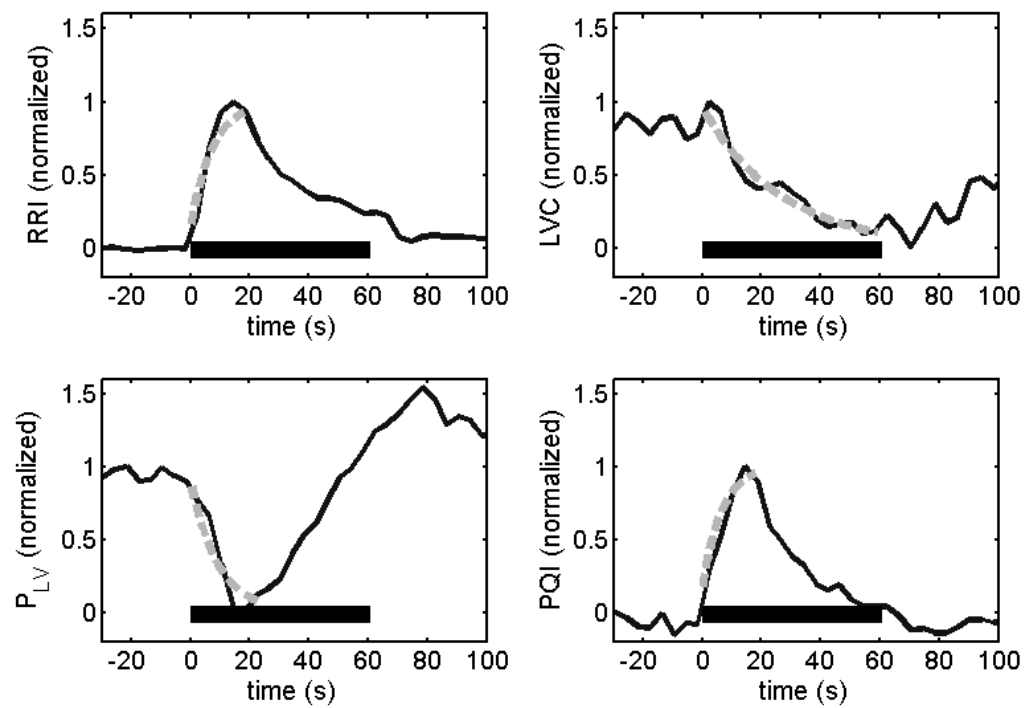

Figure 7.1: Example of the effect of vagus nerve stimulation on all parameters in the same pig. In this pig, $P_{L V}$ and PQI showed a return towards baseline while stimulation was continued. The thick horizontal line indicates the stimulation period; the dashed grey line shows the fitted exponential function.

Correlation between time constants $(\tau)$ was assessed to test whether the dynamics of the cardiovascular system were related. The $\tau$ values were significantly correlated between all cardiovascular parameters, except between LVC and PQI (table 7.1).

Table 7.1: Pearson's correlation coefficients (r) and p-values of the correlation analysis. Shaded areas indicate duplicate correlations.

\begin{tabular}{llll}
\hline & $\tau_{\mathrm{PQI}}$ & $\tau_{\mathrm{LVC}}$ & $\tau_{\mathrm{Plv}}$ \\
\hline$\tau_{\mathrm{RRI}}$ & $\mathrm{r}=0.940$ & $\mathrm{r}=0.867$ & $\mathrm{r}=0.969$ \\
& $\mathrm{p}=0.002^{*}$ & $\mathrm{p}=0.005^{*}$ & $\mathrm{p}=0.000^{*}$ \\
& $\mathrm{n}=7$ & $\mathrm{n}=8$ & $\mathrm{n}=9$ \\
\hline$\tau_{\mathrm{PQI}}$ & & $\mathrm{r}=0.729$ & $\mathrm{r}=0.900$ \\
& & $\mathrm{p}=0.100$ & $\mathrm{p}=0.006^{*}$ \\
& & $\mathrm{n}=6$ & $\mathrm{n}=7$ \\
\hline$\tau_{\mathrm{LVC}}$ & & $\mathrm{r}=0.790$ \\
& & $\mathrm{p}=0.020^{*}$ \\
& & $\mathrm{n}=8$ \\
\hline
\end{tabular}

* Correlation is significant at the 0.05 level 
A return towards baseline values during VNS was seen for LVC in two out of ten pigs, for $\mathrm{P}_{\mathrm{LV}}$ in two out of ten pigs and for PQI in four out of ten pigs (see figure 7.1 for an example). Most often it was seen in RRI, i.e. in five out of ten pigs. An exponential first order model was fitted to the return towards baseline separately. The time constant $\tau_{\text {RRI }}$ of this return in RRI $(4.8 \pm 1.8 \mathrm{~s})$ was significantly larger than $\tau_{\mathrm{RRI}}$ of the initial increase in RRI $(14.4 \pm 8.1 \mathrm{~s}$, a difference of $9.3 \pm 6.4 \mathrm{~s}, \mathrm{P}=0.03, \mathrm{n}=5$ ). Due to the low number the time constant of the return towards baseline was not statistically tested for the other cardiovascular parameters.

The three experiments with atrial pacing gave three extra values for $\tau_{\mathrm{PQI}}$ and $\tau_{\mathrm{LVC}}$ and two extra values for $\tau_{\mathrm{Plv}}$, due to a poor model fit in one pig for $\tau_{\mathrm{Plv}}$. These time constants mostly had similar values to the time constants without pacing (table 7.2), but could not be analyzed statistically due to their low number. Return towards baseline occurred for exactly the same cardiovascular parameters as when the heart was not paced.

Table 7. 2. Values of $\tau$ with and without atrial pacing.

\begin{tabular}{lll}
\hline & Without pacing & With pacing \\
\hline$\tau_{\mathrm{PQI}}$ & 3.09 & 4.27 \\
& 4.83 & 7.81 \\
& 5.93 & 8.66 \\
\hline$\tau_{\mathrm{LVC}}$ & 2.49 & 4.04 \\
& 14.96 & 10.88 \\
& & 12.66 \\
\hline$\tau_{\mathrm{Plv}}$ & 2.49 & 5.24 \\
& 9.20 & 10.50 \\
& 5.86 & \\
\hline
\end{tabular}

\subsection{Discussion}

In this chapter the effects of vagus nerve stimulation on the cardiovascular system were analyzed. We found that a return towards baseline cardiovascular parameter values can occur while left vagus nerve stimulation is continued. This was the case for LVC in two out of ten pigs, for $\mathrm{P}_{\mathrm{LV}}$ in two out of ten pigs, for PQI in four out of ten pigs, and for RRI in five out of ten pigs. A similar return towards baseline values was reported by Tosato et al. [4] to occur with right vagus nerve stimulation. Tosato did not find a return towards baseline with left vagal stimulation, possibly because they studied only two animals.

The time constant $\tau_{\text {RRI }}$ of the return towards baseline of RRI was 9.3 seconds on average larger than $\tau_{\mathrm{RRI}}$ of the initial increase in RRI, suggesting that this return towards baseline is influenced by the more slowly acting sympathetic system. 
Due to the low number the return towards baseline was not statistically tested for the other cardiovascular parameters.

The dynamics of the initial cardiovascular effects of vagus nerve stimulation, described by time constant $\tau$, were significantly correlated between all cardiovascular parameters, except between LVC and PQI. However, a significant correlation between the dynamics of LVC and PQI was found with the recordings obtained in paced animals included. This suggests that a correlation exists in baseline condition, but that the number of animals was too low to obtain a statistically significant correlation coefficient. A correlation between the cardiovascular dynamics was expected, since VNS influences all four cardiovascular parameters. It should be considered that reflexive pathways and mechanical interactions may influence the effects. For example, a change in pressure might result from a change in heart rate due to the baroreceptor reflex.

In the three pigs in which RRI was kept constant through atrial pacing, return towards baseline occurred for same parameters as when the heart was not paced. This shows that heart rate does not influence the return towards baseline effect of the other cardiovascular parameters. It also suggests that whether or not a return towards baseline occurs is subject dependent.

A first order exponential model was used to characterize the parameters during VNS. This model was an accurate fit $\left(\mathrm{R}^{2}>0.5\right)$ to the data in $87 \%$ of cases. For PQI the model was an adequate fit in only $70 \%$ of the cases. This is likely a consequence of the fact that P and Q waves have a relatively small amplitude and signal to noise ratio is therefore relatively low. This may have led to variations in PQI values and may explain why the fit to PQI of the exponential model was poor in $30 \%$ of pigs. Alternatively, the effect of vagus nerve stimulation on PQI versus time does not exhibit an exponential shape in some pigs.

\subsection{Conclusion}

A return towards baseline values can occur for all four cardiovascular parameter while left vagus nerve stimulation is maintained. The dynamics of the initial cardiovascular effects of vagus nerve stimulation are significantly correlated with each other.

\section{References}

1. Ford, T.W. and P.N. Mcwilliam (1986) The effects of electrical stimulation of myelinated and non-myelinated vagal fibres on heart rate in the rabbit J Physiol-London, 380(Nov), p. 341-7.

2. Lewis, M.E., A.H. Al-Khalidi, R.S. Bonser, T. Clutton-Brock, D. Morton, D. Paterson, J.N. Townend, and J.H. Coote (2001) Vagus nerve 
stimulation decreases left ventricular contractility in vivo in the human and pig heart J Physiol-London, 534(2), p. 547-52.

3. Schiereck, P., N. Sanna, and W.L. Mosterd (2000) Av blocking due to asynchronous vagal stimulation in rats Am J Physiol-Heart C, 278(1), p. H67-H73.

4. Tosato, M., K. Yoshida, E. Toft, and J.J. Struijk. Characterization of the cardiac response to vagal nerve stimulation. in 2 nd International IEEE EMBS Conference on Neural Engineering. 2005. Arlington, Virginia.

5. Celler, B.G. (1989) Characteristics of cardiac period responses to prolonged vagal-stimulation in dogs Med Biol Eng Comput, 27(6), p. 595-602.

6. Celler, B.G. and N.H. Lovell (1991) Dynamics of cardiac period responses after prolonged vagal-stimulation in the dog Ann Biomed Eng, 19(3), p. 273-89.

7. Warner, H.R. and R.O. Russell (1969) Effect of combined sympathetic and vagal stimulation on heart rate in dog Circ Res, 24(4), p. 567-73.

8. Randall, D.C. (1974) Concurrent measurement of left-ventricular dpdtmax, isometric contractile-force and cardiac loading in intact monkey Cardiology, 59(5), p. 304-18. 


\title{
Chapter 8
}

\section{Selectivity for specific \\ cardiovascular effects of vagus nerve stimulation with a multi-contact electrode cuff}

\author{
Simone Ordelman \\ Lilian Kornet \\ Richard Cornelussen \\ Rik Buschman \\ Peter Veltink
}

Submitted 


\section{Abstract}

The cardiovascular system can be influenced by electrically stimulating the vagus nerve. Selectivity for specific cardiac fibres may be limited when stimulating at the cervical level. Our objective was to increase effectiveness and selectivity for cardiovascular effects of vagus nerve stimulation by using local bipolar stimulation in one nerve cross-section using a multi-contact cuff instead of less localized stimulation using a tripolar ring electrode.

Both types of cuff electrodes were compared with respect to their relative effects on R-R interval (RRI), P-Q interval (PQI), left ventricular contractility $(\mathrm{LVC})$ and left ventricular pressure $\left(\mathrm{P}_{\mathrm{LV}}\right)$ in seven pigs.

Stimulation using the optimal bipolar configuration on the multi-contact cuff significantly affected RRI, PQI, LVC and $\mathrm{P}_{\mathrm{LV}}$, whereas stimulation with the ring electrode only significantly affected RRI and PQI. The cardiovascular parameters that could be significantly influenced varied between the bipolar configurations. These novel findings may be relevant for optimizing electrode configurations for clinical cardiac applications of vagus nerve stimulation. 


\subsection{Introduction}

Vagus nerve stimulation is performed clinically as a therapy for epilepsy [1], and is currently being explored for heart failure management [2-3]. Vagus nerve stimulation has also been proposed for preventing arrhythmias secondary to myocardial infarction [4] and alleviating angina pectoris [5]. When the cervical vagus nerve is electrically stimulated with bursts of stimuli, acute cardiovascular effects are a decrease in blood pressure and left ventricular contractility, and an increase in R-R interval and P-Q interval [6-8]. When aiming for specific cardiovascular effects, limited selectivity of vagus nerve stimulation can be a problem. Efferent cardiac fibres have a small range of diameters [9], which means that multiple cardiovascular parameters will be influenced simultaneously. Fibres of a larger diameter than the desired cardiac fibres may be blocked by quasi-trapezoidal pulses [10]. However, it may be difficult to design a pulse that selectively and completely blocks a particular set of fibres, due to different cross-sectional positions of the myelinated fibres in the vagus nerve [10]. A possible solution is to improve spatial selectivity, i.e. to localize the current to the fibres of interest.

Spatial selectivity characteristics of a nerve cuff depend on electrode configurations. Cuffs with multiple ring contacts along the nerve lead to activation of a large area within the nerve. Multi-contact cuffs with local bipolar stimulation in one cross-section result in improved selective activation of bundles of fibres in the periphery of the nerve, close to the cathode [11-13]. This approach has not been evaluated for selective stimulation of the vagus nerve to induce specific cardiac effects. Spatial selectivity may be achieved by using a steering anode with a longitudinal tripole [12] or a steering anode with a cathodic monopole [14]. We chose to use a cuff with bipolar stimulation between adjacent electrodes in one cross section. We designed a multi-contact electrode cuff for this purpose and compared it to a tripolar ring configuration. We hypothesized that local bipolar stimulation induces larger cardiovascular effects compared to stimulation with a tripolar ring cuff. Also, we hypothesized that with bipolar stimulation cardiovascular parameters can be influenced selectively and independently of each other.

\subsection{Methods}

\subsubsection{Experimental setup}

Vagus nerve stimulation and sensing experiments were performed in seven female Dutch Landrace pigs. Animal handling was performed according to the Dutch Law and the European Directive for the Protection of Animals and was approved by the Experimental Animal Committee of the Maastricht University. The pig was premedicated with $0.5 \mathrm{mg} / \mathrm{kg}$ Morphine 20 minutes before induction with $3 \mathrm{mg} / \mathrm{kg}$ Zoletil (Telazol) and $0.5 \mathrm{mg} / \mathrm{kg}$ xylazine. The pig was 
anesthetized with a combination of isoflurane $(1-1.5 \%)$ and fentanyl (6-10 $\mu \mathrm{g} / \mathrm{kg} / \mathrm{hr}$ ). The left vagus nerve was carefully dissected free from the surrounding tissue at the cervical level and two custom-made self-sizing cuff electrode configurations were placed for stimulation. One configuration was a cuff with rings, $15 \mathrm{~mm}$ long with three circular Pt/Ir electrode contacts with an interelectrode distance of $4 \mathrm{~mm}$ (figure 8.1a). The other configuration was a multi-contact cuff in which the three rings were each replaced by four rectangular electrodes (figure 8.1b). The surface area of the square contacts was approximately $2 \mathrm{~mm}^{2}$. The cuffs were made in three diameters $(2,3.5$ and 5 $\mathrm{mm}$ ), to ensure a proper fit around the nerve (not too tight to avoid nerve damage and not too loose for optimal electrical contact between the electrode and the nerve), and even spacing of the electrode contacts of the multi-contact cuff at $90^{\circ}$ increments. The cuff was designed with twelve contacts for sensing purposes, but only the contacts on the middle ring were used in this study.
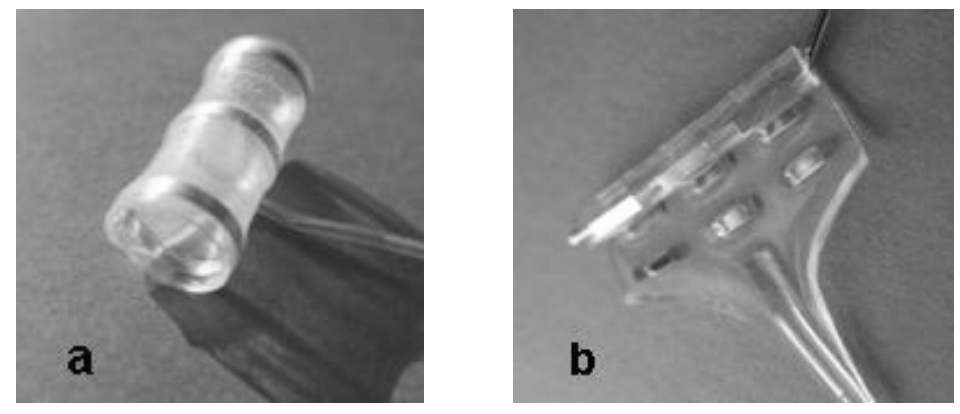

Figure 8.1: a) Ring electrode cuff. b) multi-contact electrode cuff

Surface electrodes were placed on shaved paws for recording the ECG. Left ventricular blood pressure was measured with a Millar catheter (Millar Instruments, Houston, Texas, USA). ECG and blood pressure recordings were amplified with a TMSi Porti amplifier (TMS international, Oldenzaal, The Netherlands). The ECG and blood pressure were sampled at $2048 \mathrm{~Hz}$. Data was stored on a laptop using PortiLab2 software (TMS international, Oldenzaal, The Netherlands).

The vagus nerve was stimulated using biphasic pulses generated by a custommade stimulator. The second pulse had an exponential shape and a relatively low amplitude with respect to the first so as not to influence recruitment importantly, yet guarantee zero net charge transfer through the electrode-tissue interface. Stimulation was performed with a first phase pulse width of $300 \mu$ s, a pulse amplitude range of 1 to $10 \mathrm{~mA}$, and a stimulation frequency range of 10 to $50 \mathrm{~Hz}$. Burst stimulation was maintained for 60 seconds, or until side effects occurred (such as complete heart block or a digestive reflex). The stimulation amplitude and frequency that elicited the highest decrease in heart rate with the 
ring cuff were determined. In each pig, stimulation was repeated five times with these stimulation parameters, i.e. one time with the ring cuff where the middle ring was the cathode and the outer two rings were anodes, and four times with the multi-contact cuff where the four contacts of the middle ring were consecutively used as a cathode with one radially adjacent contact as the anode (figure 8.2).

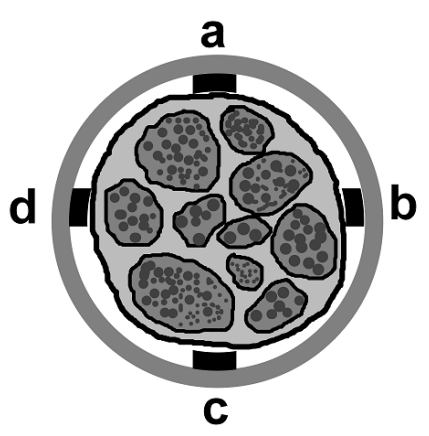

Figure 8.2: Schematic representation of the multi-contact electrode cuff around a nerve, looking in caudal direction. Stimulation was performed using two adjacent electrode sites as cathode and anode respectively. Four combinations were possible: $a-b, b-c, c-d$ and $d-a$.

\subsubsection{Signal analysis}

Offline signal analysis was performed in Matlab R2009a (The Mathworks Inc.). $\mathrm{P}, \mathrm{Q}$ and R-tops of the ECG were detected automatically and confirmed by visual inspection. R-R and P-Q intervals were determined. Left ventricular contractility was assessed using maximum values of the time derivative of left ventricular pressure: $\left(\frac{\mathrm{dP}_{\mathrm{LV}}}{\mathrm{dt}}\right)_{\max }[15]$. This parameter will be called left ventricular contractility $(\mathrm{LVC})$. The systolic left ventricular pressures $\left(\mathrm{P}_{\mathrm{LV}}\right)$ were determined as the maximum values in each cardiac cycle of a pressure recording.

The effect on a cardiovascular parameter was determined as a percentage increase or decrease from the baseline value. We measured baseline values of the cardiovascular parameters for at least two breathing cycles before onset of stimulation. After stimulation was started we waited until the cardiovascular parameters had stabilized to determine the effect from an average of two breathing cycles. If the effect did not stabilize, but returned towards baseline value while stimulation was maintained, we averaged a window of two breathing cycles around the peak effect. 
The bipolar configuration on the multi-contact cuff with the largest effect on RRI, PQI, LVC, and $\mathrm{P}_{\mathrm{LV}}$ respectively was determined for each pig as the optimal bipolar configuration for the respective parameters. The largest effect was the largest increase for RRI and PQI, and the largest decrease for LVC and $\mathrm{P}_{\mathrm{LV}}$, based on the direction of the effects as reported in the literature [6-8].

\subsubsection{Statistical analysis}

Data was tested for normality with a Shapiro-Wilk test. Normally distributed data is presented as mean \pm standard deviation, other data as median (interquartile range). The effects of stimulation were tested for significance with a one-sample Student's t-test for normally distributed data and with a sign test otherwise. A paired-sample t-test was used to test the difference between the effect obtained with the ring cuff and the optimal bipolar configuration on the multi-contact cuff.

It was tested if stimulation with the optimal bipolar configuration of the multicontact cuff for one cardiovascular parameter also affected the other parameters significantly at the $\mathrm{p}=0.05$ level, by combining the results from all pigs.

The cardiovascular effects were evaluated in seven anesthetized pigs. All data was normally distributed, except for the effects on PQI when stimulating with the multi-contact cuff.

In all pigs, the cardiovascular system could be affected by burst stimulation. The effects came about directly at onset of stimulation and gradually increased when bust stimulation was maintained. A plateau was reached within the 60 seconds of stimulation. A return towards baseline while stimulation was maintained occurred in five out of seven pigs for PQI, in four out of seven pigs for RRI, and in one out of seven pigs for $\mathrm{LVC}$ and $\mathrm{P}_{\mathrm{LV}}$.

In a small number of pigs, the cardiovascular effects were positive for some configurations and negative for others. This was the case in one out of seven pigs for RRI and LVC, and in two out of seven pigs for $\mathrm{P}_{\mathrm{LV}}$.

Stimulation using the optimal bipolar configuration on the multi-contact cuff significantly affected RRI, PQI, LVC and $\mathrm{P}_{\mathrm{LV}}$, whereas stimulation with the tripolar ring electrode using the same stimulation parameters significantly affected only RRI and PQI (see table 8.1). An example of the effect on RRI with the ring cuff and the multi-contact cuff is shown in figure 8.3. The effect observed using the multi-contact cuff was significantly larger than that observed using the ring for the parameter LVC $(-10 \pm 7 \%$ versus $-1 \pm 11 \%$ respectively $\mathrm{P}=0.047$ ), but not for the other cardiac parameters RRI, $\mathrm{P}_{\mathrm{LV}}$ and PQI. 
Table 8.1: Percentage value and statistical significance of the effect of stimulation using the ring cuff, and using the optimal cathode-anode configuration on the multi-contact cuff. $N=7$.

\begin{tabular}{l|l|l|l}
$\begin{array}{l}\text { Cardiovascular } \\
\text { parameter }\end{array}$ & $\begin{array}{l}\text { Effect using the ring } \\
\text { cuff }(\%)\end{array}$ & $\begin{array}{l}\text { Effect using the } \\
\text { optimal } \\
\text { configuration on the } \\
\text { multi-contact cuff } \\
(\%)\end{array}$ & $\begin{array}{l}\text { Difference between } \\
\text { the two cuffs (p- } \\
\text { value from paired t- } \\
\text { test })\end{array}$ \\
\hline \hline RRI & $\begin{array}{l}10 \pm 8 \\
(p=0.02)\end{array}$ & $\begin{array}{l}18 \pm 17 \\
(p=0.03)\end{array}$ & $\begin{array}{l}9 \pm 19 \\
(p=0.275)\end{array}$ \\
\hline PQI & $\begin{array}{l}15 \pm 10 \\
(p=0.01)\end{array}$ & $\begin{array}{l}17(16) \\
(p=0.02)\end{array}$ & $\begin{array}{l}4 \pm 7 \\
(p=0.639)\end{array}$ \\
\hline LVC & $\begin{array}{l}-1 \pm 11 \\
(p=0.81)\end{array}$ & $\begin{array}{l}-10 \pm 7 \\
(p=0.01)\end{array}$ & $\begin{array}{l}-9 \pm 9 \\
(p=0.126)\end{array}$ \\
\hline$P_{\mathrm{LV}}$ & $-1 \pm 15$ & $\begin{array}{l}-11 \pm 7 \\
(p=0.89)\end{array}$ & $\begin{array}{l}-10 \pm 16 \\
(\boldsymbol{p}=0.047)\end{array}$
\end{tabular}

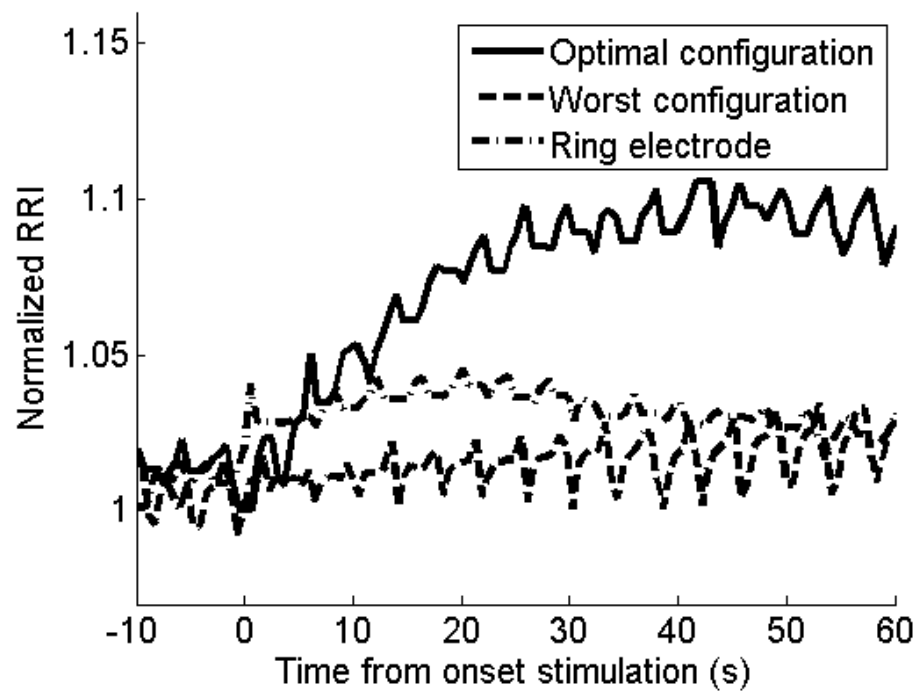

Figure 8.3: Example of the effect on RRI of electrical stimulation with the ring electrode (-.), the optimal bipolar configuration on the multi-contact cuff (-) and the worst bipolar configuration on the multi-contact cuff (--). Cyclic variations are relating to the breathing cycle, which was kept constant with a ventilator. 
The effects of stimulation with the optimal bipolar configurations for the four cardiovascular parameters are presented in table 8.2. When stimulating with the optimal bipolar configuration for affecting RRI, parameter LVC is not significantly affected and vice versa. When the optimal bipolar configuration for affecting PQI is selected, no significant effect is observed on LVC. However, when stimulating for affecting LVC, PQI is also significantly affected. When the optimal bipolar configuration for affecting $\mathrm{P}_{\mathrm{LV}}$ is used for stimulation, no significant effect was observed on RRI or PQI. However, there is no parameter that can be influenced without affecting $\mathrm{P}_{\mathrm{LV}}$.

Table 8.2: Independent activation of the cardiovascular effects when stimulating with the multi-contact electrode. $N=7$.

\begin{tabular}{|c|c|c|c|c|}
\hline $\begin{array}{l}\text { Cardiovascular parameter for which } \\
\text { the optimal cathode-anode } \\
\text { configuration is selected }\end{array}$ & $\begin{array}{l}\text { Effect on } \\
\text { RRI }(\%)\end{array}$ & $\begin{array}{l}\text { Effect on } \\
\mathrm{PQI}(\%)\end{array}$ & $\begin{array}{l}\text { Effect on } \\
\text { LVC (\%) }\end{array}$ & $\begin{array}{l}\text { Effect on } \\
\text { PLV }(\%)\end{array}$ \\
\hline RRI & $\begin{array}{l}18 \pm 17 \\
(p=0.03)\end{array}$ & $\begin{array}{l}3(17) \\
(p=0.03)\end{array}$ & $\begin{array}{l}-4 \pm 11 \\
(p=0.37)\end{array}$ & $\begin{array}{l}-8 \pm 6 \\
(p=0.01)\end{array}$ \\
\hline $\mathrm{PQI}$ & $\begin{array}{l}13(12) \\
(p=0.02)\end{array}$ & $\begin{array}{l}17(16) \\
(p=0.02)\end{array}$ & $\begin{array}{l}-8 \pm 9 \\
(p=0.05)\end{array}$ & $\begin{array}{l}-6 \pm 5 \\
(p=0.01)\end{array}$ \\
\hline LVC & $\begin{array}{l}11 \pm 16 \\
(p=0.14)\end{array}$ & $\begin{array}{l}10 \pm 9 \\
(p=0.03)\end{array}$ & $\begin{array}{l}-10 \pm 7 \\
(p=0.01)\end{array}$ & $\begin{array}{l}-11 \pm 7 \\
(p=0.01)\end{array}$ \\
\hline $\mathrm{P}_{\mathrm{LV}}$ & $\begin{array}{l}11 \pm 18 \\
(p=0.15)\end{array}$ & $\begin{array}{l}3(17) \\
(p=0.06)\end{array}$ & $\begin{array}{l}-9 \pm 8 \\
(p=0.02)\end{array}$ & $\begin{array}{l}-11 \pm 7 \\
(p=0.004)\end{array}$ \\
\hline
\end{tabular}

\subsection{Discussion}

Improved effectiveness in vagal stimulation for cardiovascular effects was obtained with the multi-contact cuff compared to the ring cuff. Significant effects on RRI, PQI, LVC and $\mathrm{P}_{\mathrm{LV}}$ were obtained when stimulating with the multi-contact cuff, whereas stimulation with the ring cuff only gave significant effects on RRI and PQI. Also, the effect on LVC was significantly larger with the multi-contact cuff than with the ring cuff. This is consonant with our hypothesis that local bipolar stimulation in one nerve cross-section is more effective for obtaining cardiovascular effects than stimulation with a tripolar ring cuff configuration along the nerve.

When selecting a bipolar configuration from the multi-contact cuff for stimulation, a subset of the four cardiovascular parameters is affected. This implies that the nerve fibres innervating the sinus node, the ventricle wall and the AV node have different spatial arrangements in the vagus nerve. For example, if efferent fibres influencing RRI and PQI are arranged within the 
same fascicle, they will be activated simultaneously due to their location with respect to the stimulating cathode. If efferent fibres influencing RRI and LVC are arranged in fascicles on different sides of the nerve, they may be activated separately. In future studies, histological analysis should be included after each experiment to provide a link between cross sectional fibre position and cardiovascular effects.

If a cardiovascular parameters cannot be influenced without an effect on other cardiovascular parameters, these parameters my be physiologically related. For example, a change in pressure might be the result of a change in heart rate due to the baroreceptor reflex. It is estimated that central processing of baroreceptor afferent activity may require 0.25 second in the human [16]. Thus, in our experiments where we measure the maximum change in a 60 second timeframe, a change in pressure might be induced by a change in heart rate. Also, a change in heart rate is expected to change LVC, because the Frank Starling mechanism regulates that more filling due to a slower heart rate is compensated by a higher contractility [17]. Thus in case vagal stimulation increases RRI as we found, we would expect an increase in LVC solely due to the effect of heart rate on LVC. These and other mechanisms may explain the simultaneous effects on cardiovascular parameters.

Spatial selectivity may be further improved by decreasing the activated area of the nerve. Increasing the number of bipolar configurations around the circumference of the nerve could attain this. However, the number of bipolar configurations that can be placed around the circumference of a nerve is limited. To maintain the self-coiling ability, four contacts could maximally be placed radially in our cuff. Placing the contacts closer together will also lead to a larger leakage of current to the layer between the cuff and the nerve [11].

When using electrodes with a small surface area - such as a multi-contact cuff for electrical stimulation, electrode corrosion and tissue damage are potential risks. Established electrochemical safety limits for platinum electrodes range from $0.1 \mathrm{mC} . \mathrm{cm}^{-2}$ [18] to $0.4 \mathrm{mC} . \mathrm{cm}^{-2}$ [19]. Our electrodes on the multi-contact cuff have a surface area of about $2 \mathrm{~mm}^{2}$. With the pulse width of $300 \mu$ s used in our study, stimulus amplitude should not exceed $7 \mathrm{~mA}$ to $27 \mathrm{~mA}$. If higher amplitudes are needed, electrodes with a larger surface area should be used. A larger surface area can be obtained without compromising selectivity by increasing the electrode surface roughness [20].

Safety is expected not to be a problem with our cuff electrode design. Other studies have demonstrated that cuff electrodes are safe for long term implantation [21-23]. For example, self-coiling spiral-cuffs were implanted on the hypoglossal nerve in the dog for up to 17 months and no functional damage was observed [22]. 
The electrode design we used in this study is just one of many configurations proposed for selective stimulation in the literature. Other cuff designs that improve spatial selectivity are also potentially useful for cardiovascular selectivity. For example, the FINE electrode [24] flattens the nerve within a cuff to reposition the fascicles with respect to the electrode contacts. Intraneural electrodes such as the longitudinally implanted intrafascicular electrode (LIFE) [25] may also improve selectivity by delivering a current directly into the fascicles of interest.

Female pigs were used in this study. This may have affected our results, since gender differences are known to exist in human autonomic cardiovascular regulation [26]. Variations in the oestrous cycle phases of the pigs used in our experiments may also have influenced our results, because of the hormonal influence on vagus nerve activity [27]. Future studies should be performed in a larger number of female and male pigs to eliminate this variability.

This study is the first to demonstrate that a multi-contact electrode cuff with local bipolar stimulation can be used to target specific cardiovascular parameters in vagus nerve stimulation. This novel finding should be taken into account when optimizing electrode configurations for clinical cardiac applications of vagus nerve stimulation. Potentially, the selectivity properties of a multi-contact cuff can also be advantageous for avoiding side effects of electrical stimulation, such as coughing. This needs to be studied in awake animals.

\subsection{Conclusion}

More significant cardiovascular effects of vagal stimulation can be obtained with a multi-contact cuff compared to a ring cuff, at the same stimulation level. The effect on LVC was significantly larger with a multi-contact cuff than with a ring cuff. When the optimal bipolar configuration is used for a certain parameter, not all other cardiovascular parameters are significantly influenced.

\subsection{Acknowledgements}

We would like to acknowledge Paul van Venrooij, who designed and Joost Ubachs who manufactured the electrode cuffs used in this study.

\section{References}

1. Chadwick, D. (2001) Vagal-nerve stimulation for epilepsy Lancet, 357(9270), p. 1726-7.

2. Schwartz, P.J. and G.M. De Ferrari (2009) Vagal stimulation for heart failure: Background and first in-man study Heart Rhythm, 6(11), p. S76-S81. 
3. Zhang, Y.H., Z.B. Popovic, S. Bibevski, I. Fakhry, D.A. Sica, D.R. Van Wagoner, and T.N. Mazgalev (2009) Chronic vagus nerve stimulation improves autonomic control and attenuates systemic inflammation and heart failure progression in a canine high-rate pacing model Circulation-Heart Failure, 2(6), p. 692-9.

4. Vanoli, E., G.M. Deferrari, M. Strambabadiale, S.S. Hull, R.D. Foreman, and P.J. Schwartz (1991) Vagal-stimulation and prevention of sudden-death in conscious dogs with a healed myocardial-infarction Circ Res, 68(5), p. 1471-81.

5. Bilgutay, A.M., I.M. Bilgutay, F.K. Merkel, and C.W. Lillehei (1968) Vagal tuning. A new concept in the treatment of supraventricular arrhythmias, angina pectoris, and heart failure $\mathrm{J}$ Thorac Cardiovasc Surg, 56(1), p. 71-82.

6. Ford, T.W. and P.N. Mcwilliam (1986) The effects of electrical stimulation of myelinated and nonmyelinated vagal fibres on heart rate in the rabbit J Physiol-London, 380(Nov), p. 341-7.

7. Lewis, M.E., A.H. Al-Khalidi, R.S. Bonser, T. Clutton-Brock, D. Morton, D. Paterson, J.N. Townend, and J.H. Coote (2001) Vagus nerve stimulation decreases left ventricular contractility in vivo in the human and pig heart J Physiol-London, 534(2), p. 547-52.

8. Schiereck, P., N. Sanna, and W.L. Mosterd (2000) Av blocking due to asynchronous vagal stimulation in rats Am J Physiol-Heart C, 278(1), p. H67-H73.

9. Agostoni, E., J.E. Chinnock, M.D.B. Daly, and J.G. Murray (1957) Functional and histological studies of the vagus nerve and its branches to the heart, lungs and abdominal viscera in the cat $\mathrm{J}$ Physiol-London, 135(1), p. 182-205.

10. Tosato, M., K. Yoshida, E. Toft, and J.J. Struijk (2007) Quasitrapezoidal pulses to selectively block the activation of intrinsic laryngeal muscles during vagal nerve stimulation J Neural Eng, 4(3), p. 205-12.

11. Deurloo, K.E.I., J. Holsheimer, and H.B.K. Boom (1998) Transverse tripolar stimulation of peripheral nerve: A modelling study of spatial selectivity Med Biol Eng Comput, 36(1), p. 66-74.

12. Sweeney, J.D., D.A. Ksienski, and J.T. Mortimer (1990) A nerve cuff technique for selective excitation of peripheral-nerve trunk regions IEEE T Bio-Med Eng, 37(7), p. 706-15.

13. Veraart, C., W.M. Grill, and J.T. Mortimer (1993) Selective control of muscle activation with a multipolar nerve cuff electrode IEEE T BioMed Eng, 40(7), p. 640-53.

14. Tarler, M.D. and J.T. Mortimer (2004) Selective and independent activation of four motor fascicles using a four contact nerve-cuff electrode IEEE Trans Neural Syst Rehabil Eng, 12(2), p. 251-7. 
15. Randall, D.C. (1974) Concurrent measurement of left-ventricular dpdtmax, isometric contractile-force and cardiac loading in intact monkey Cardiology, 59(5), p. 304-18.

16. Borst, C. and J.M. Karemaker (1983) Time delays in the human baroreceptor reflex J Auton Nerv Syst, 9(2-3), p. 399-409.

17. Yadid, M., G. Sela, D. Amiad Pavlov, and A. Landesberg (2011) Adaptive control of cardiac contraction to changes in loading: From theory of sarcomere dynamics to whole-heart function Pflugers Arch, 462(1), p. 49-60.

18. Robblee, L.S. and T.L. Rose, Electrochemical guidelines for selection of protocols and electrode materials for neural stimulation. Neural prostheses: Fundamental studies, ed. W.F. Agnew and D.B. McCreery. 1990, Englewood Cliffs, NJ: Prentice Hall.

19. Brummer, S.B. and M.J. Turner (1977) Electrical-stimulation with pt electrodes .2. Estimation of maximum surface redox (theoretical nongassing) limits IEEE T Bio-Med Eng, 24(5), p. 440-3.

20. Merrill, D.R., M. Bikson, and J.G.R. Jefferys (2005) Electrical stimulation of excitable tissue: Design of efficacious and safe protocols Journal of Neuroscience Methods, 141(2), p. 171-98.

21. Polasek, K.H., H.A. Hoyen, M.W. Keith, R.F. Kirsch, and D.J. Tyler (2009) Stimulation stability and selectivity of chronically implanted multicontact nerve cuff electrodes in the human upper extremity IEEE Trans Neural Syst Rehabil Eng, 17(5), p. 428-37.

22. Sahin, M., D.M. Durand, and M.A. Haxhiu (1999) Chronic recordings of hypoglossal nerve activity in a dog model of upper airway obstruction Journal of Applied Physiology, 87(6), p. 2197-206.

23. Schwartz, P.J., G.M. De Ferrari, A. Sanzo, M. Landolina, R. Rordorf, C. Raineri, C. Campana, M. Revera, N. Ajmone-Marsan, L. Tavazzi, and A. Odero (2008) Long term vagal stimulation in patients with advanced heart failure: First experience in man Eur J Heart Fail, 10(9), p. 884-91.

24. Tyler, D.J. and D.D.M. Durand (2002) Functionally selective peripheral nerve stimulation with a flat interface nerve electrode IEEE T Neur Sys Reh, 10(4), p. 294-303.

25. Zheng, X.J., M. Zhang, T.Y. Chen, and Z.W. Chen (2008) Recording and stimulating properties of chronically implanted longitudinal intrafascicular electrodes in peripheral fascicles in an animal model Microsurgery, 28(3), p. 203-9.

26. Evans, J.M., M.G. Ziegler, A.R. Patwardhan, J.B. Ott, C.S. Kim, F.M. Leonelli, and C.F. Knapp (2001) Gender differences in autonomic cardiovascular regulation: Spectral, hormonal, and hemodynamic indexes J Appl Physiol, 91(6), p. 2611-8.

27. Goldman, R.K., A.S. Azar, J.M. Mulvaney, C. Hinojosa-Laborde, J.R. Haywood, and V.L. Brooks (2009) Baroreflex sensitivity varies during 
the rat estrous cycle: Role of gonadal steroids Am J Physiol Regul Integr Comp Physiol, 296(5), p. R1419-26. 


\section{Chapter 9}

\section{Vagus nerve recording and stimulation in awake pigs}




\section{Abstract}

Experiments of vagus nerve recording and stimulation in the awake animal were performed to obtain results that are more representative of the clinical condition than the anesthetized experiments. The cardiac-modulated pattern and the stimulation thresholds for side effect were analyzed.

A cardiac-modulated pattern was found in six out of ten pigs. The shape and amplitude of the pattern differed from the anesthetized condition, but no consistent or significant differences were found. The stimulation threshold for side effect was significantly higher with the multi-contact cuff than with the ring electrode.

We conclude that cardiac-modulated patterns can be recorded from awake animals, and that side effects may be reduced or avoided by using a multicontact cuff. 


\subsection{Introduction}

In this chapter, experiments of vagus nerve recording and stimulation in the awake animal are described. The awake condition is more representative of the clinical condition than the anesthetized condition, in which suppressive effects of anaesthetics are present [1]. In the awake condition movements may influence the recordings, and side effects may occur such as hoarseness, throat pain and coughing [2]. These factors are relevant to consider when designing a system for clinical application.

In the awake condition the cardiac-modulated pattern as described in chapter two has not yet been inspected. It is crucial to know if it can also be observed when the animal is awake, and if it is different from the anesthetized condition. This will give further insight into the feasibility of using the cardiac-modulated neural activity as an input for a closed-loop vagus nerve stimulator. Recording from the vagus nerve in awake animals has been presented in the literature before. Experiments in awake goats have shown a relationship between amplitude in vagal activity and blood pressure [3-4].

Vagus nerve stimulation has been clinically evaluated for epilepsy and depression $[2,5]$. Recently, clinical studies of vagus nerve stimulation for heart failure have been performed [6-8]. For these clinical applications, stimulation parameters are relatively low, at amplitudes below $3.5 \mathrm{~mA}$, and stimulation frequency typically below $30 \mathrm{~Hz}$ [5]. The acute cardiovascular effects that we have studied in chapter seven occurred at higher levels, with amplitudes between $5 \mathrm{~mA}$ and $10 \mathrm{~mA}$, and frequency up to $50 \mathrm{~Hz}$. The acute cardiovascular effects at these settings have not been reported in awake animals in the literature before. We would like to study what these effects are, for their possible application in vagus nerve therapy for cardiovascular disease.

Vagus nerve stimulation at higher levels may lead to side effects such as coughing, hoarseness and throat pain. These side effects already occur at the lower stimulation parameters used in clinical VNS for epilepsy, depression and heart failure $[2,5,7]$. We therefore also expect to observe them in our experiments for acute cardiovascular effects in awake animals. One possible way to avoid side effects may be the use of spatial selectivity of the multisegment cuff, as described in chapter eight. Spatial selectivity may limit the activation of fibres relating to side effects, by delivering the current to only part of the nerve.

The aim of this chapter is to compare the results of vagus nerve stimulation and recording experiments in awake animals to those in the anesthetized condition reported in previous chapters. We analyze the cardiac-modulated pattern in the spontaneous neural activity, and look at acute effects of vagus nerve stimulation 
on the cardiovascular system. We also inspect whether thresholds for side effects vary between electrode configurations.

\subsection{Methods}

The experiments were performed in ten female Dutch Landrace pigs. The pig was premedicated with $0.5 \mathrm{mg} / \mathrm{kg}$ Morphine 20 minutes before induction with $3 \mathrm{mg} / \mathrm{kg}$ Zoletil (Telazol) and $0.5 \mathrm{mg} / \mathrm{kg}$ xylazine. The pig was anesthetized with a combination of isoflurane $(1-1.5 \%)$ and fentanyl $(6-10 \mu \mathrm{g} / \mathrm{kg} / \mathrm{hr})$. The left vagus nerve was dissected free from surrounding tissue at the cervical level. Two cuff electrodes with three circular electrode sites were used for electrical stimulation and recording respectively, with the recording cuff cranial to the stimulation cuff. Cranial to these two cuffs, a multi-segment electrode cuff was placed that had three rings of four square contacts (chapter eight). The connectors of the cuff electrodes were tunnelled under the skin to the back of the neck, where the connectors exited through the skin. The wound was then closed. The connectors were placed in a plastic pouch and attached with cotton strings to the cotton stretch jacket that was put on the pig's torso. The pigs were awoken and allowed to recover overnight.

Starting the next day, while the pig was awake, experiments were performed for seven consecutive days. During these measurements, the pig was placed into a wheeled cage for constraint (figure 9.1). ECG electrodes were placed on shaved paws for recording ECG. The connectors of the cuff electrodes were removed from the plastic pouch and attached to the amplifier cables (figure 9.2). Impedance of all electrodes was measured daily. An increase in impedance could indicate diminishing experimental conditions, such as damage to the electrode wires or decreased contact between the electrode and the nerve.

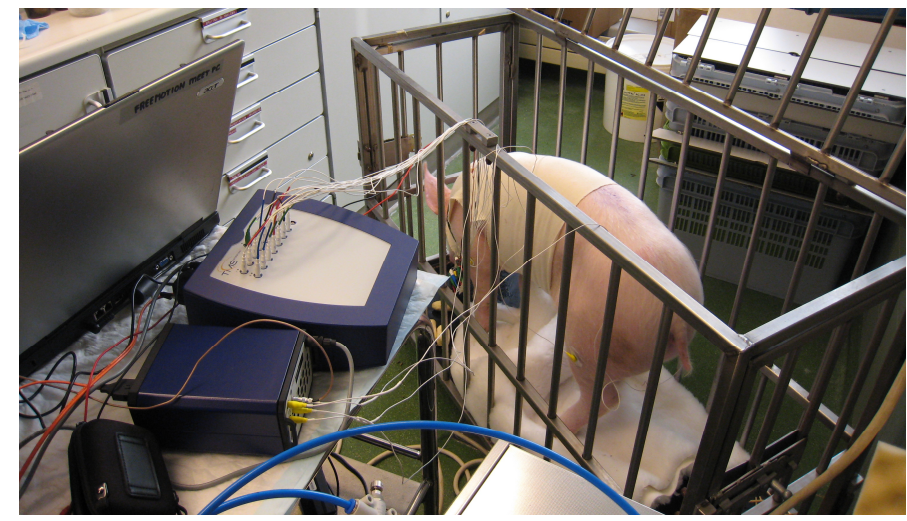

Figure 9.1: experimental setup with the pig placed in a wheeled cage. The connectors from the cuffs and the ECG electrodes are connected to the amplifiers 


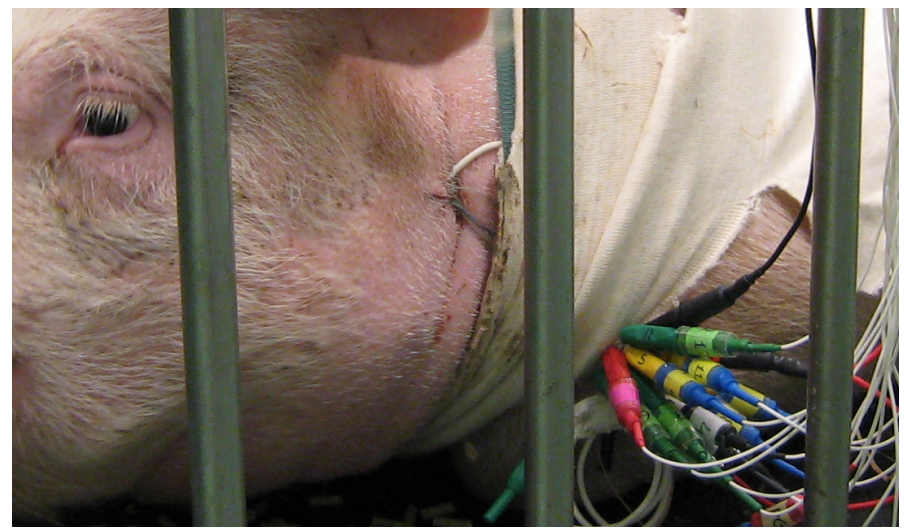

Figure 9.2: Close-up of the electrode wires exiting the skin and the electrode connectors attached to the amplifier cables

Spontaneous vagal activity and ECG were recorded for five minutes. Next, electrical nerve stimulation was performed with a custom-made stimulator, with a train of 30 individual pulses $(1 \mathrm{~Hz}, 300 \mu \mathrm{s}, 0.1-10 \mathrm{~mA})$. This stimulation was performed on the most caudal ring electrode cuff and consecutively on four cathode-anode configurations of the multi-contact cuff, as described in chapter eight. The amplitude was increased $(0.05 \mathrm{~mA}, 0.1 \mathrm{~mA}, 0.2 \mathrm{~mA}, 0.5 \mathrm{~mA}, 1 \mathrm{~mA}$, $1.5 \mathrm{~mA}, 2 \mathrm{~mA}, 3 \mathrm{~mA}, 5 \mathrm{~mA}, 10 \mathrm{~mA})$ until side effects occurred such as coughing, or the pig was thought to experience discomfort from the stimulation. The stimulation setting at which this occurred was noted. Impedance measurements were used to verify that there was a good contact between the electrode and the tissue and no wire damage. Stimulation frequency was then increased from 1 to $50 \mathrm{~Hz}$, in an attempt to influence heart rate and PQ interval by vagus nerve stimulation. The ECG was monitored during these trials. Stimulation was stopped as soon as the pig showed signs of side effects or discomfort.

Neural recordings were amplified with a TMSi Refa amplifier, and ECG signals with a TMSi Porti amplifier (both TMS international, Oldenzaal, The Netherlands). The data were sampled at $20 \mathrm{kHz}$ and stored on a laptop using PortiLab2 software (TMS international, Oldenzaal, The Netherlands).

The signals were analyzed offline in Matlab R2007a (The Mathworks Inc.). Signal analysis for cardiac-modulated patterns in the spontaneous vagal activity is described in the methods section of chapter two. If data was available for more than one day, data analysis was performed on the data of the first day when experimental conditions were expected to be best. 
After the seven days of awake experiments, the vagus nerve was dissected free again and the cuffs were inspected. It was noted if damage to the electrodes was observed, and if the cuffs had come loose from the nerve.

\subsection{Results}

In six out of ten pigs $(60 \%)$ a cardiac-modulated pattern could be recorded in the awake pigs, compared to nine out of the same ten pigs $(90 \%)$ in the anesthetized condition (chapter two). Five out of the six pigs that showed a cardiac-modulated pattern when recorded during the awake state had also shown a cardiac-modulated pattern in the anesthetized condition. However, the electrode on the multi-segment cuff that showed the most pronounced modulation was different between the two conditions in both pigs. The phase of increased activity also differed between the awake and anesthetized condition (table 9.1, figure 9.3). The average amplitude of the vagal activity after filtering $\left(\mathrm{V}_{\mathrm{avr}}\right)$ in the awake condition was not significantly different from the anesthetized condition.

Table 9.1: Overview of the difference in cardiac-modulated signals between the awake and the anesthetized condition. Shaded areas indicate when no data is available.

\begin{tabular}{c|c|c|c|c|c|c} 
Pig & \multicolumn{2}{|c|}{ Phase of modulation } & \multicolumn{2}{c|}{ Amplitude $\mathbf{V}_{\text {avr }}(\boldsymbol{\mu} \mathbf{V})$} & \multicolumn{2}{c}{ Heart rate (bpm) } \\
\hline \hline & Awake & Anesthetized & Awake & Anesthetized & Awake & Anesthetized \\
\hline \hline $\mathbf{1}$ & TP & QRST \& TP & 1.5731 & 1.7806 & 145 & 119 \\
\hline $\mathbf{2}$ & QRST & & 1.6088 & & 123 & \\
\hline $\mathbf{3}$ & & & & & & \\
\hline $\mathbf{4}$ & TP & TP & 1.5873 & 1.7364 & 138 & 103 \\
\hline $\mathbf{5}$ & QRST & TP & 1.6022 & 2.2855 & 140 & 85 \\
\hline $\mathbf{6}$ & & & & & & \\
\hline $\mathbf{7}$ & & & & & & \\
\hline $\mathbf{8}$ & TP/PQ & QRST & 1.4521 & 1.6845 & 126 & 101 \\
\hline $\mathbf{9}$ & & & & & & \\
\hline $\mathbf{1 0}$ & QRST & PQ & 1.6829 & 1.4062 & 146 & 108
\end{tabular}

Burst stimulation to influence the heart could not be performed in the awake experiments. It became apparent that stimulation amplitude and frequency could not be increased high enough to influence the heart. Before a stimulation setting was found that decreased heart rate, the pigs started coughing or showed signs of discomfort, such as grunting or startled movements, and the experiments were halted. Heart rate never decreased by more than five percent during a stimulation experiment. 

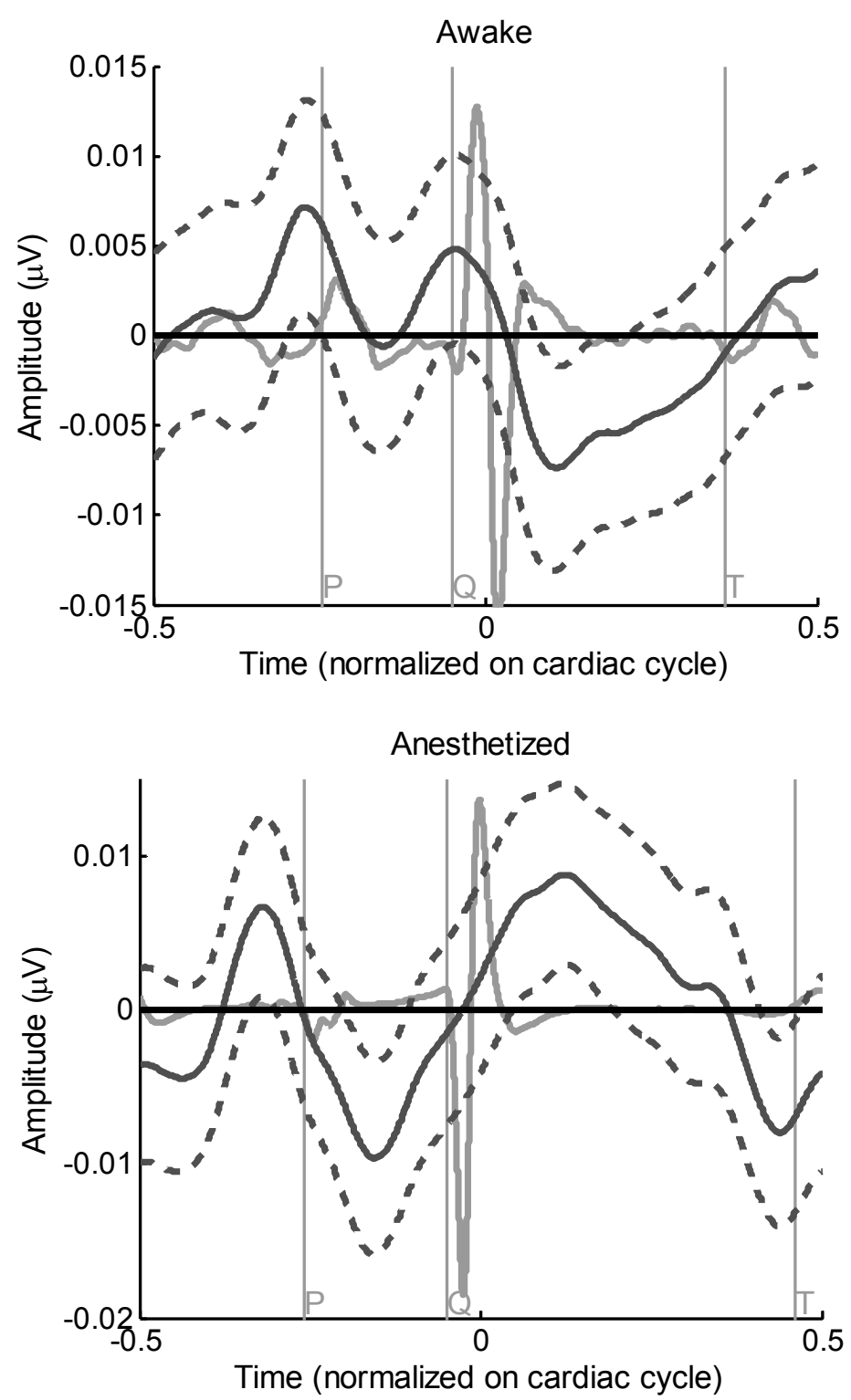

Figure 9.3: Modulation from the same pig, when awake (above) and anesthetized (below). Time is normalized on the average cardiac cycle length. The signals are from two different electrodes, since the electrode that showed the modulation differed between the two conditions. The pattern of the modulation also differs, with increased activity in the TP phase when awake and in the TP and QRST phase when anesthetized. 
The threshold for side effects could be determined in five out of ten pigs using both the ring cuff and the multi-contact cuff (table 9.2). At least one cathodeanode configuration on the multi-contact cuff corresponded to a higher threshold for side effects than the ring electrode in all five pigs (figure 9.4; Wilcoxon Signed Rank test $(\mathrm{P}<0.05 ; \mathrm{n}=5))$. In all five pigs the observed side effect was coughing. The threshold when using the ring electrode cuff was found at $1 \mathrm{~mA}$ in all five pigs. In two pigs the highest threshold was $2 \mathrm{~mA}$. In the other three pigs, at one or more cathode-anode configurations, coughing did not occur up to $10 \mathrm{~mA}$ and the threshold was therefore set at $10 \mathrm{~mA}$. In the five pigs in which side effects were determined impedance measurements confirmed that there was a good contact between the electrode and the tissue and no wire damage preventing current from being delivered to the nerve. Effects of stimulation, such as compound action potentials or decreased heart rate, could not be observed to verify that the nerve was excited, both in recordings that elicited a coughing response and in those that did not. In the pigs in which no thresholds for side effects could be analyzed, the impedance was too high or connectors were damaged on the first day.

Table 9.2: Overview of the ten pigs. It is indicated for each pig on which day a high

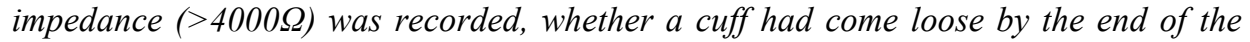
experiment, whether a cardiac-modulated signal could be recorded while the pig was awake and whether thresholds for side effects were determined.

\begin{tabular}{c|c|c|c|c} 
Pig & $\begin{array}{c}\text { High } \\
\text { impedance }\end{array}$ & $\begin{array}{c}\text { Cuffs loose } \\
\text { from nerve }\end{array}$ & $\begin{array}{c}\text { Cardiac- } \\
\text { modulated } \\
\text { signal }\end{array}$ & $\begin{array}{c}\text { Thresholds for } \\
\text { side effects } \\
\text { determined }\end{array}$ \\
\hline \hline $\mathbf{1}$ & Day 1 & All three & Yes & No \\
\hline $\mathbf{2}$ & Day 1 & $\begin{array}{c}\text { Multi-contact } \\
\text { cuff }\end{array}$ & Yes & No \\
\hline $\mathbf{3}$ & Day 5 & None & No & No \\
\hline $\mathbf{4}$ & Day 4 & None & Yes & Yes \\
\hline $\mathbf{5}$ & No & $\begin{array}{c}\text { Multi-contact } \\
\text { cuff }\end{array}$ & Yes & Yes \\
\hline $\mathbf{6}$ & No & $\begin{array}{c}\text { Multi-contact } \\
\text { cuff }\end{array}$ & No & Yes \\
\hline $\mathbf{7}$ & Day 3 & All three & No & Yes \\
\hline $\mathbf{8}$ & No & None & Yes & No \\
\hline $\mathbf{9}$ & No & None & No & No \\
\hline $\mathbf{1 0}$ & No & None & Yes & Yes
\end{tabular}


The difference in threshold amplitude for coughing between the multi-contact cathode-anode configuration with the highest and the lowest threshold was up to $8 \mathrm{~mA}(\mathrm{P}<0.1 ; \mathrm{n}=5$; Wilcoxon Signed Rank test, figure 9.4). The stimulation threshold for coughing varied between configurations of the multi-contact cuff in four out of five pigs. In the fifth pig, no threshold for coughing was found with the multi-contact cuff at the highest amplitude, and the threshold was estimated at $10 \mathrm{~mA}$ for all configurations.

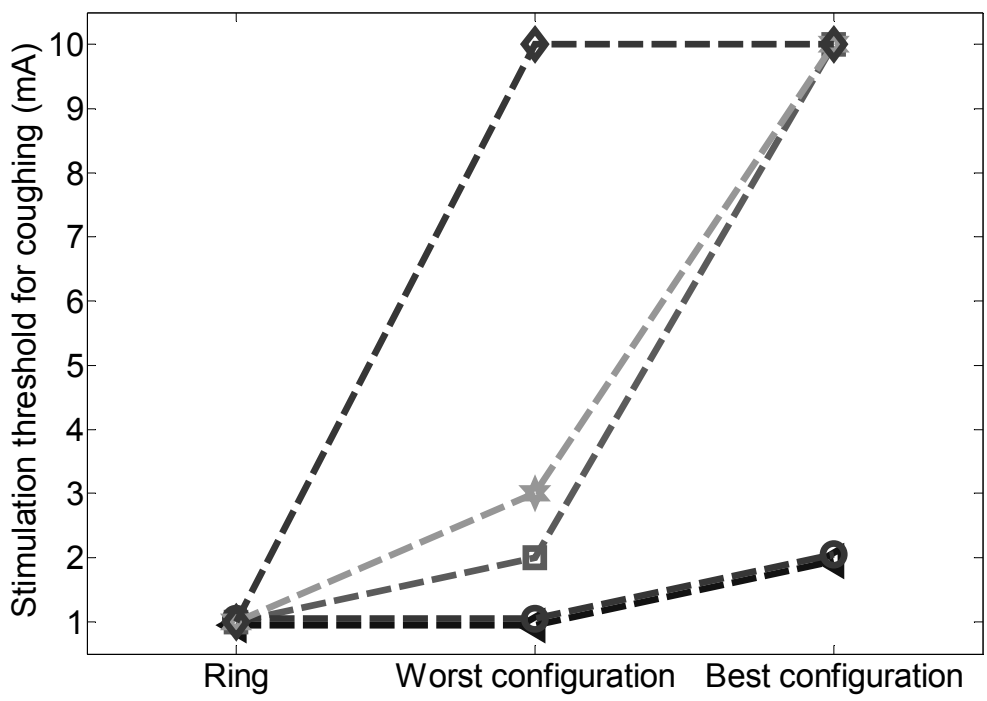

Figure 9.4: The stimulation threshold in $m A$ at which coughing occurred when stimulating with the ring cuff (left), with the cathode-anode configuration on the multicontact electrode cuff with the lowest threshold (centre), or with the highest threshold (right). Results from five pigs.

Problems arose with the electrode connectors. The plastic pouch had come loose from the connectors in all pigs; therefore the connectors were not sufficiently protected. In all pigs, one or multiple connectors detached from the wires at some point during the seven days of awake experiments. In five out of ten pigs high impedance $(>4000 \Omega)$ was measured after one to five days (table 9.2). In three out of these five pigs, one or more of the cuff electrodes had come loose from the nerve before the end of the seven days. For two out of ten pigs, one cuff had come loose from the nerve by the time of inspection (after the seven days of awake recording), but no substantial increase of impedance was observed during the awake experiments. 


\subsection{Discussion}

The cardiac-modulated patterns found in the awake pigs differed from the patterns found when these same pigs were anesthetized. This may be caused by shifting of the electrode cuffs. Shifting can cause other fibre populations to be recorded, which would also lead to a difference in the pattern of the recorded signal. This could also explain why a different electrode contact showed the most pronounced modulation. The average amplitude of the cardiac-modulated vagal activity was expected to be increased in the awake pig compared to the anesthetized condition, because anaesthetics have suppressive effects [1]. No conclusions about vagal signal amplitude can be drawn from this small data set.

Our results show that, as hypothesized, the threshold at which side effects occur can be influenced by spatial selectivity. Selecting the optimal cathode-anode configuration for stimulation can increase the threshold amplitude. However, with our setup it was not possible to avoid side effects at stimulation levels needed for cardiac effects. Even though amplitudes up to $10 \mathrm{~mA}$ could be delivered at $1 \mathrm{~Hz}$ without side effects, when stimulation frequency was increased to levels that were expected to influence the heart rate or PQ interval $(50 \mathrm{~Hz}$, based on results such as presented in chapter seven), side effects did occur. Frequency of chronic vagus nerve stimulation for heart failure is lower, for example one stimulation pulse per heart beat [6], and may therefore be achieved without the side effect of coughing using the multi-contact cuff. Side effects could also be avoided by using strategies such as anodal blocking, in which a nerve fibre is hyperpolarized near an anode, such that large diameter fibres can be blocked [9]. It should be investigated whether cardiovascular effects still remain with this technique. It should also be noted that the occurrence and intensity of side effects often diminishes over time with vagus nerve stimulation [10].

The absence of side effects at high stimulation amplitudes could be caused by a lack of fibre excitation. This may be the case when the electrode has come loose from the nerve. Impedance measurements were used to verify that there was a good contact between the electrode and the tissue and no wire damage preventing current from being delivered to the nerve. However, only if an effect of stimulation is observed, such as a CAP or a decrease in heart rate, can it be ascertained that the stimulation current was delivered to the nerve. Only it that case can one be certain that the threshold for side effects is indeed higher than the highest tested amplitude. We were never able to observe cardiovascular effects of vagus nerve stimulation or record CAPs when testing the thresholds for side effects. This however does not mean that there was no current being delivered to the nerve, since it is also possible that the recording of CAPs was the problem as was also the case in the experiments in which coughing occurred while no CAPs were recorded. 
Cardiac-modulated patterns were found in six out of ten pigs, which is a lower number than the nine out of ten in the anesthetized pigs. This may be caused by damage to the electrodes or artefacts from tension to the wires. Other possible explanations are damage to the nerve itself or the formation of tissue or blood cloths between the electrode and the nerve.

Comparing the cardiac-modulated patterns between the awake and anesthetized condition did not provide a significant difference in amplitude or phase of increased activity. Heart rate was higher during the awake experiments, but no blood pressure measurements could be performed. Blood pressure measurements are more useful to link the recorded signal to the cardiac receptors, since we expect to measure signals from pressure receptors. Comparing awake and anesthetized measurements is complicated due to the large time in-between recordings, in which electrodes may have shifted, leading to a different population of fibres that are recorded from. A larger data set may also be needed to observe differences between the two conditions.

Damage to the electrodes occurred, which was primarily caused by external forces such as rolling and chafing of the pig. The problem of external forces may be addressed by using a sturdier jacket for the pig, with pockets for the connectors. Also, the type of connector should be improved upon, to avoid them coming loose from the wires. One plug for all the connectors combined could provide a more stable solution. Alternatively, wireless systems that use radio frequency transmission to an external receiver, e.g. [11-12], could be used to avoid tension to the wires and damage to the electrode contacts. Reduced quality of contact between the electrodes and the nerve may in addition be prevented by coating the electrodes to prevent infection and scar tissue forming [13-15]. Additional awake experiments with these suggested improvements are needed.

\section{References}

1. Urban, B.W. (2002) Current assessment of targets and theories of anaesthesia Br J Anaesth, 89(1), p. 167-83.

2. $\quad$ Morris, G.L., 3rd and W.M. Mueller (1999) Long-term treatment with vagus nerve stimulation in patients with refractory epilepsy. The vagus nerve stimulation study group e01-e05 Neurology, 53(8), p. 1731-5.

3. Yambe, T., S. Nanka, S. Kobayashi, A. Tanaka, M. Yoshizawa, K. Abe, K. Tabayashi, H. Takeda, and S. Nitta (1999) Vagal nerve activity recording in the awake condition for the control of an artificial heart system Artif Organs, 23(6), p. 529-31.

4. Yambe, T., S.S. Nanka, Y. Shiraishi, A. Tanaka, M. Yoshizawa, K. Abe, K. Tabayashi, H. Takeda, and S. Nitta (2003) Recording vagal nerve activity for the control of an artificial heart system Asaio J, 49(6), p. 698-700. 
5. Labiner, D.M. and G.L. Ahern (2007) Vagus nerve stimulation therapy in depression and epilepsy: Therapeutic parameter settings Acta Neurol Scand, 115(1), p. 23-33.

6. De Ferrari, G.M., H.J. Crijns, M. Borggrefe, G. Milasinovic, J. Smid, M. Zabel, A. Gavazzi, A. Sanzo, R. Dennert, J. Kuschyk, S. Raspopovic, H. Klein, K. Swedberg, and P.J. Schwartz (2011) Chronic vagus nerve stimulation: A new and promising therapeutic approach for chronic heart failure Eur Heart J, 32(7), p. 847-55.

7. Schwartz, P.J. and G.M. De Ferrari (2009) Vagal stimulation for heart failure: Background and first in-man study Heart Rhythm, 6(11), p. S76-S81.

8. Schwartz, P.J., G.M. De Ferrari, A. Sanzo, M. Landolina, R. Rordorf, C. Raineri, C. Campana, M. Revera, N. Ajmone-Marsan, L. Tavazzi, and A. Odero (2008) Long term vagal stimulation in patients with advanced heart failure first experience in man Eur J Heart Fail, 10(9), p. 884-91.

9. Vuckovic, A., M. Tosato, and J.J. Struijk (2008) A comparative study of three techniques for diameter selective fibre activation in the vagal nerve: Anodal block, depolarizing prepulses and slowly rising pulses $\mathrm{J}$ Neural Eng, 5(3), p. 275-86.

10. Ben-Menachem, E. (2001) Vagus nerve stimulation, side effects, and long-term safety J Clin Neurophysiol, 18(5), p. 415-8.

11. Obeid, I., M.A. Nicolelis, and P.D. Wolf (2004) A multichannel telemetry system for single unit neural recordings J Neurosci Methods, 133(1-2), p. 33-8.

12. Szuts, T.A., V. Fadeyev, S. Kachiguine, A. Sher, M.V. Grivich, M. Agrochao, P. Hottowy, W. Dabrowski, E.V. Lubenov, A.G. Siapas, N. Uchida, A.M. Litke, and M. Meister (2011) A wireless multi-channel neural amplifier for freely moving animals Nat Neurosci, 14(2), p. 2639.

13. He, W., G.C. McConnell, T.M. Schneider, and R.V. Bellamkonda (2007) A novel anti-inflammatory surface for neural electrodes Advanced Materials, 19(21), p. 3529-33.

14. Keefer, E.W., B.R. Botterman, M.I. Romero, A.F. Rossi, and G.W. Gross (2008) Carbon nanotube coating improves neuronal recordings Nature Nanotechnology, 3(7), p. 434-9.

15. Lu, Y., D.F. Wang, T. Li, X.Q. Zhao, Y.L. Cao, H.X. Yang, and Y.Y. Duan (2009) Poly(vinyl alcohol)/poly(acrylic acid) hydrogel coatings for improving electrode-neural tissue interface Biomaterials, 30(25), $\mathrm{p}$. 4143-51. 


\section{Chapter 10}

General discussion 


\subsection{Introduction}

This thesis focused on sensing cardiovascular signals from the vagus nerve and stimulating the vagus nerve for cardiovascular effects. The first part of this thesis described sensing the vagus nerve. A cardiac-modulated vagus nerve activity pattern was found in chapter two, which may reflect cardiac receptor activity. Evoked vagus nerve responses were analyzed in anesthetized animals in chapter three, and again in chapter six for awake animals. Responses were found that possibly relate to neurocardiac control loops. Chapter four uses a computer model to investigate whether it is possible to record an evoked dispersed component, such as described in chapter three. In chapter five the evoked responses are further analyzed in two additional experiments and an involvement of the recurrent laryngeal nerve was found. Chapter six shows that evoked vagus nerve responses from the brainstem may be recorded in awake pigs or when using intraneural needle electrode.

The second part of this thesis focused on electrical stimulation of the vagus nerve. Chapter seven showed that burst vagus nerve stimulation has various cardiac effects, which can be affected by reflexive mechanisms. The results of chapter eight show that selectivity for cardiovascular effects can be improved by using a segmented electrode configuration. In chapter nine experiments for vagus nerve sensing and stimulation in awake animals are described, and the challenges that present themselves in the awake condition are presented.

This final chapter discusses the results presented in this thesis. These results are also interpreted for their possible merit for closed loop neurocardiac control. Finally, some future perspectives are discussed and the final conclusions are presented.

All experiments were performed in pigs, since these animals are a good model for humans concerning the neural and cardiovascular system. We chose to approach the vagus nerve in the neck region, because at this location it can be reached easily in surgery, as is also done for implantation of a vagus nerve stimulator in epilepsy patients. Reaching the vagus nerve closer to the heart would require open chest surgery, with accompanying risks for the patient.

\subsection{Sensing cardiovascular signals from the vagus nerve}

\subsubsection{Spontaneous vagus nerve activity}

Spontaneous neural activity was recorded from the vagus nerve in order to investigate whether it contains cardiac-modulated patterns (chapter two). It was concluded that a neural activity pattern can be found that is modulated with the cardiac cycle, when recording from the whole vagus nerve in the neck region of anesthetized pigs. This pattern changed when blood pressure and ventricular contractility were increased due to dobutamine administration, which indicated 
that changes in cardiac receptor firing are reflected in the recorded cardiacmodulated neural signals. These experiments were repeated in awake animals (chapter nine), in which a similar cardiac-modulated pattern was observed. The fact that cardiac-modulated vagal signals can be recorded in awake animals suggests that it may be feasible to record such signals from patients, as is the eventual goal. Spontaneous vagal activity may be useful for monitoring cardiovascular parameters. Even though the direction of the signal could not be determined, we can assume that the recorded cardiac-modulated pattern is an afferent signal, since it is known from single-fibre recordings that afferent vagal signals are modulated with the cardiac cycle [1-6]. In addition to cardiovascular parameters such as blood pressure and contractility, afferent cardiac activity can also be related to pathological states such as ischemia [7-8]. Recording spontaneous activity for monitoring purposes would be possible when simultaneously electrically stimulating the vagus nerve, if the stimulation frequency is set low enough to record spontaneous neural activity between pulses. Alternatively, monitoring on one side of the neck and electrically stimulating on the other side may be a solution.

Two electrode cuffs were compared for recording spontaneous neural activity from the vagus nerve. One was a cuff with three ring electrodes that record from the whole perimeter of the nerve. The other was a cuff with 12 segments, i.e. four segments around the perimeter of the nerve, used in an attempt to improve recording of cardiac-modulated patterns by improving spatial selectivity. This cuff with 12 segments was indeed more effective than the cuff with rings for recording the cardiac-modulated patterns. Therefore, a cuff with multiple segments of some sort should be used when attempting to monitor the cardiovascular system through spontaneous vagus nerve activity. Variations on this cuff design, in number of segments or overall cuff design, should be tested for optimization. Techniques such as beamforming spatial filtering algorithms may also be used to extract relevant signals [9-10].

\subsubsection{Evoked vagus nerve responses}

The evoked vagus nerve responses were analyzed in an attempt to find components related to neurocardiac control, either efferent from the brain stem or afferent from the neural network on the heart. A dispersed, fast component was found that possibly came from the neural network on the heart (chapter three). This component was related to cardiovascular parameters through its latency and dispersion, which indicated that it came from the neural network on the heart. The model presented in chapter four showed that neural signal similar to the component could be recreated with the model when using a dispersed burst of single fibre action potentials that is non-randomly generated. However, the fact that the simulated component remained on the centre electrode, whereas the component recorded in the pigs was strongly reduced, shows that the hypothesis of a burst of single fibre action potentials did not match the origin of 
the experimentally observed component adequately. The strongly decreased signal on a tripolar configuration is an argument for its muscular origin, since tripolar derivations are known to reduce muscular interference. Additional experiments showed that the recurrent laryngeal nerve was somehow involved in the component, and the original hypothesis needed to be revised. As described in chapter five, the component may be from cardiac neural fibres travelling via the recurrent laryngeal nerve, or it may be interference from evoked muscular laryngeal activity. At this point, it remains unclear if the component is of neural or muscular origin.

Efferent evoked vagus nerve components, coming from the brainstem, may have been observed in awake animals or when using needle electrodes (chapter six). This needs to be more solidly established in further studies. Such components may be related to the level of vagal reflexive sensitivity. This may for example be used to assess baroreflex sensitivity, which is known to predict outcome in heart failure [11-13]. Whether the efferent evoked components can indeed be used in such a way needs to be further examined. The origin of the components needs to be elucidated and their relation to baroreflex sensitivity made apparent.

Other evoked signals may be present that have not been recorded with our current setup. The results of an evoked efferent component as described in chapter six, which was only recorded by intraneural needle electrodes, suggest that components may have been missed because they are obscured by the other signals in the vagus nerve. Experiments could be performed that record from cardiac branches close to the heart to identify such components. The results described in chapter six in awake pigs suggest that anaesthesia may have suppressed efferent reflexive components, and this also needs further investigation.

Chronic monitoring of evoked components can be done when electrical stimulation is performed chronically for therapeutic purposes, as may be the case for a heart failure application. Electrical stimulation at low levels, i.e. subthreshold for noticeable effects, may be used to monitor evoked responses when therapeutic effects of vagus nerve stimulation are not desired. Whether this is possible depends on the stimulation level required for generating the desired evoked responses relative to the level needed for therapeutic stimulation. As mentioned before, when using a combination of spontaneous vagus nerve activity and electrical stimulation, the time between electrical stimuli (and following evoked responses) should be large enough to not interfere with the analysis of the spontaneous activity. 


\subsubsection{The average reference method}

The use of the average reference method for recording neural activity has shown both benefits and disadvantages over the more commonly used tripolar derivation. As described in chapter two, the average reference configuration improves the odds of recording a cardiac-modulated signal in spontaneous vagus nerve activity. This suggests that this is the optimal method when monitoring the spontaneous vagal activity. The major disadvantage of the average reference method is that it does not suppress muscular interferences from outside of the cuff as effectively as a tripolar derivation does. This was however not apparent in the signal to noise ratio when comparing the middle ring electrode (which is also a tripolar derivation) to the outer ring electrodes, in neither the anesthetized pigs (chapter two) nor the awake pigs (chapter nine). Possibly, little muscular interference was present, or the cuff was not closed perfectly to create an ideal situation for reducing muscular interference.

Since there is no advantage of the average reference method when recording evoked responses to be used as feedback, a tripolar derivation may be the most sensible case in such a system. However, if velocity and direction of the evoked responses should be determined to identify and classify the evoked neural responses for feedback purposes, a single tripolar configuration does not suffice. A configuration with multiple electrodes along the nerve is needed for velocityselective filtering [14]. Direction and velocity of signals can be determined within a multiple electrode cuff, by dividing the time it takes for a signal to travel from one electrode to the next by the distance between the two electrodes. This could be achieved with multiple tripolar configurations [13], but would then require at least one more electrode than with the average reference configuration. Space along the nerve is generally limited by anatomical constraints, which makes the average reference method a better choice. In addition, the average reference method allows for subsequent spatial filtering when cuffs with multiple segmented electrodes around the perimeter are used. However, evoked muscular activity may disturb the recordings when using the average reference method.

\subsection{Electrical vagus nerve stimulation for cardiovascular effects}

\subsubsection{Possible benefits}

Electrical stimulation of the vagus nerve is beneficial in heart failure, in cases in which medication is not sufficient [15]. It has a positive effect on cardiac parameters and quality of life. It may also prevent the deteriorating effects of low vagal tone. Vagus nerve stimulation for heart failure has been shown to be safe, tolerable and feasible [16-17]. At this time the understanding of the working mechanisms of vagus nerve stimulation for heart failure is incomplete. The beneficial effects of VNS may be attributed to several different 
mechanisms [18]: A reduced heart rate, increased heart rate variability, improved baroreflex sensitivity, direct antiarrhythmic effects, or antiinflammatory effects.

Both the left and right vagus nerve was stimulated in the pilot experiments of our study. Electrical stimulation of the right vagus nerve elicited little to no effect on cardiovascular parameters, which led to the decision to focus on the left vagus nerve. According to the literature, right vagus nerve stimulation should elicit an effect on heart rate [19], which is inconsistent with our findings. This discrepancy could be explained by the fact that we studied effects of vagus nerve stimulation in intact animals [20], in which reflexive loops may influence the cardiovascular effects.

\subsubsection{Electrode design}

In our study a cuff electrode is used for electrical stimulation because it can be used chronically, is safe, and delivers the current locally. Selectivity of electrical stimulation may be improved by adapting cuff design, such as described in chapter eight for spatial selectivity. In this chapter, it was attempted to modify not only the level of the effect, but also the type of effect. This could lead to a more advanced therapy, which may especially be suited to adapt to the diverse aspects of heart failure ranging from ischemia to heart rhythm complications. Since multiple mechanisms may be at play in the positive effects of vagus nerve stimulation for heart failure, each of these mechanisms may be beneficial for specific situations. It may be possible to activate these mechanisms separately using spatial selectivity, leading to specialized stimulation settings for selected events. It may also be possible to use the benefits of spatial selectivity for other disease states, such as arrhythmias, high blood pressure, epilepsy, depression or obesity.

\subsection{Closed-loop neurocardiac control}

A closed loop system for artificial neurocardiac control has potential benefits over constant level vagus nerve stimulation. Acute events can be handled acutely to avoid adverse outcomes. For example, an increase in stimulation may be needed for arrhythmias or ischemia, whereas stimulation needs to be decreased when the cardiovascular system is inhibited too much. Also, side effects may be avoided if stimulation is reduced when high levels are not needed.

A healthy body integrates many processes for neurocardiac control, whereas in this thesis the focus is only on the vagal aspect. The suggested artificial neurocardiac control system should thus be viewed as an addition to the neurocardiac control functions of the body itself, and not a replacement. The challenge is to find where the body itself is lacking and how to respond in a 
manner that restores normal functioning. Even though the complete mechanisms and interactions of neurocardiac control may remain uncertain for a long time, it has been shown that the vagus nerve has a vital role in this pathology [21-23]. An artificial system for neurocardiac control may be used to respond when this defect causes an unhealthy situation. Artificial neurocardiac control may also be beneficial in conjunction with a cardiac pacemaker, heart medication release, or inflammation regulation.

Using neural signals as feedback for neurocardiac control has the advantage of using signals that contain information about a range of cardiovascular parameters, relevant to neurocardiac control. The status of the neurocardiac signal can be monitored directly, and certain events may even be predicted and therefore avoided. Using heart cycle duration (RR interval) as feedback for closed-loop neurocardiac control has been evaluated in the literature. Bilgutay et al. [24] used vagus nerve stimulation that was automatically switched on when RR interval increased above a certain threshold value. Zhang et al. [25] used RR interval feedback for reducing the ventricular rate in dogs with AF. Tosato et al. were able to maintain a $18 \%$ change of the RR interval duration by using the heart rate as feedback variable [26]. In human studies of vagus nerve stimulation for heart failure the Cardiofit system uses an intracardiac lead to detect heart rate [17]. A limit is set at 55 beats per minute to interrupt vagal stimulation whenever heart rate became too low. Using RR interval as feedback is an obvious choice, since it can easily be derived from ECG recordings. However, it is a more limited approach than using neural signals that can, potentially, provide information about several other relevant physical and chemical quantities. Therefore, feedback control derived from neural signals may lead to more advanced closed-loop neurocardiac control such as proposed in the previous paragraphs.

\subsection{Future directions}

Before the findings of this thesis can be implemented into a clinical device, some remaining questions need to be answered. First of all, the link between cardiac vagal signals and diseased states needs to be elucidated. This may be done by repeating the experiments described in this thesis in animals with heart failure symptoms. An example of such a heart failure model is the rapid pacing model in dogs, in which the ventricles are paced at a high rated for a sustained period of time [27-29]. Experiments in awake animals, such as described in chapter nine, should also be expanded upon.

Some alternative methods may be investigated to improve stimulation performance. Our method for selective vagus nerve stimulation for cardiac effects is limited by fibre distribution characteristics of the cervical vagus nerve, as described in chapter eight. Selectivity can be improved when stimulating the 
parasympathetic system at a different location, where variation of fibre type is limited. For example, selective cardiac effects can be achieved by stimulating discrete fat pads. Different fat pads can be stimulated to selectively slow sinus rate [30], block AV node conduction [19, 31-33], or influence the ventricles [31, 33]. It needs to be studied whether fat pad stimulation is advantageous over cervical vagus nerve stimulation for cardiac diseased states, and how this can be achieved in a minimally invasive way.

It is of importance to verify the results obtained in animal studies in humans. The recording of cardiac vagal signals at the cervical level in humans should be the first step. These experiments could be performed during implantation surgery of a vagus nerve stimulator in epilepsy patients. Cuff electrodes of similar design to the ones used in our animal experiments can be used on human vagus nerve. Spontaneous activity recorded for a few minutes is enough to provide insight in the cardiac-modulated pattern in baseline condition. Evoked CAPs can be determined at low stimulation levels, allowing a comparison between the components observed in pigs and humans. If these studies show that cardiac vagal signals can be obtained from humans as from pigs, the next step could be to sense these signals in heart failure patients. Clinical studies for vagus nerve implants in heart failure patients are currently being performed [16, 34]. Future studies for vagus nerve implants in heart failure could be combined with the recording of vagal signals, in conjunction with cardiovascular parameters.

\subsection{Conclusions}

This thesis has shown that relevant cardiac signals can be obtained from the cervical vagus nerve. The impact of electrode cuff design was shown, with respect to both sensing and stimulating. The average reference configuration was presented as a new method for peripheral nerve recording, with improved performance for recording cardiac-modulated signals. Questions remain as to the origin of signals evoked by vagal stimulation. The results of this thesis can be used in the development of monitoring systems, electrical stimulation systems, or systems for closed-loop neurocardiac control.

\section{References}

1. Arndt, J.O., P. Brambring, K. Hindorf, and M. Rohnelt (1971) Afferent impulse traffic from atrial a-type receptors in cats - does a-type receptor signal heart rate Pflug Arch Eur J Phy, 326(4), p. 300-15.

2. Arndt, J.O., P. Brambring, K. Hindorf, and M. Rohnelt (1974) Afferent discharge pattern of atrial mechanoreceptors in cat during sinusoidal stretch of atrial strips insitu J Physiol-London, 240(1), p. 33-52.

3. Brown, A.M. (1965) Mechanoreceptors in or near the coronary arteries J Physiol, 177, p. 203-14. 
4. Geiger, S.R., Handbook of physiology: A critical, comprehensive presentation of physiological knowledge and concepts. Section 2: The cardiovascular system. Vol. 3. 1983, Washington: American Physiological Society.

5. Paintal, A.S. (1955) A study of ventricular pressure receptors and their role in the bezold reflex Q J Exp Physiol Cogn Med Sci, 40(4), p. 34863.

6. Thoren, P.N. (1976) Atrial receptors with nonmedullated vagal afferents in the cat. Discharge frequency and pattern in relation to atrial pressure Circ Res, 38(5), p. 357-62.

7. Cerati, D. and P.J. Schwartz (1991) Single cardiac vagal fibre activity, acute myocardial ischemia, and risk for sudden death Circ Res, 69(5), p. 1389-401.

8. Recordati, G., P.J. Schwartz, M. Pagani, A. Malliani, and A.M. Brown (1971) Activation of cardiac vagal receptors during myocardial ischemia Experientia, 27(12), p. 1423-4.

9. Wodlinger, B. and D.M. Durand (2009) Localization and recovery of peripheral neural sources with beamforming algorithms IEEE Trans Neural Syst Rehabil Eng, 17(5), p. 461-8.

10. Wodlinger, B. and D.M. Durand, Recovery of neural activity from nerve cuff electrodes, in 33rd Annual International Conference of the IEEE $E M B S$. 2011: Boston, Massachusetts USA.

11. La Rovere, M.T., G.D. Pinna, R. Maestri, E. Robbi, A. Caporotondi, G. Guazzotti, P. Sleight, and O. Febo (2009) Prognostic implications of baroreflex sensitivity in heart failure patients in the beta-blocking era $\mathrm{J}$ Am Coll Cardiol, 53(2), p. 193-9.

12. Mortara, A., M.T. La Rovere, G.D. Pinna, A. Prpa, R. Maestri, O. Febo, M. Pozzoli, C. Opasich, and L. Tavazzi (1997) Arterial baroreflex modulation of heart rate in chronic heart failure: Clinical and hemodynamic correlates and prognostic implications Circulation, 96(10), p. 3450-8.

13. Osterziel, K.J., D. Hanlein, R. Willenbrock, C. Eichhorn, F. Luft, and R. Dietz (1995) Baroreflex sensitivity and cardiovascular mortality in patients with mild to moderate heart failure $\mathrm{Br}$ Heart J, 73(6), p. 51722.

14. Taylor, J., N. Donaldson, and J. Winter (2004) Multiple-electrode nerve cuffs for low-velocity and velocity-selective neural recording Med Biol Eng Comput, 42(5), p. 634-43.

15. Piterman, L., H. Zimmet, H. Krum, A. Tonkin, and J. Yallop (2005) Chronic heart failure--optimising care in general practice Aust Fam Physician, 34(7), p. 547-53.

16. De Ferrari, G.M., H.J. Crijns, M. Borggrefe, G. Milasinovic, J. Smid, M. Zabel, A. Gavazzi, A. Sanzo, R. Dennert, J. Kuschyk, S. Raspopovic, H. Klein, K. Swedberg, and P.J. Schwartz) Chronic vagus 
nerve stimulation: A new and promising therapeutic approach for chronic heart failure Eur Heart J.

17. Schwartz, P.J., G.M. De Ferrari, A. Sanzo, M. Landolina, R. Rordorf, C. Raineri, C. Campana, M. Revera, N. Ajmone-Marsan, L. Tavazzi, and A. Odero (2008) Long term vagal stimulation in patients with advanced heart failure: First experience in man Eur J Heart Fail, 10(9), p. 884-91.

18. Olshansky, B., H.N. Sabbah, P.J. Hauptman, and W.S. Colucci (2008) Parasympathetic nervous system and heart failure: Pathophysiology and potential implications for therapy Circulation, 118(8), p. 863-71.

19. Ardell, J.L. and W.C. Randall (1986) Selective vagal innervation of sinoatrial and atrioventricular nodes in canine heart Am J Physiol, 251(4 Pt 2), p. H764-73.

20. Tosato, M., K. Yoshida, E. Toft, and J.J. Struijk. Characterization of the cardiac response to vagal nerve stimulation. in 2 nd international IEEE EMBS. 2005. Arlington, Virginia.

21. Bibevski, S. and M.E. Dunlap (2011) Evidence for impaired vagus nerve activity in heart failure Heart Fail Rev, 16(2), p. 129-35.

22. Hauptman, P.J. and D.L. Mann (2011) The vagus nerve and autonomic imbalance in heart failure: Past, present, and future Heart Fail Rev, 16(2), p. 97-9.

23. Li, W. and B. Olshansky (2011) Inflammatory cytokines and nitric oxide in heart failure and potential modulation by vagus nerve stimulation Heart Fail Rev, 16(2), p. 137-45.

24. Bilgutay, A.M., I.M. Bilgutay, F.K. Merkel, and C.W. Lillehei (1968) Vagal tuning. A new concept in the treatment of supraventricular arrhythmias, angina pectoris, and heart failure $\mathrm{J}$ Thorac Cardiovasc Surg, 56(1), p. 71-82.

25. Zhang, Y., K.A. Mowrey, S. Zhuang, D.W. Wallick, Z.B. Popovic, and T.N. Mazgalev (2002) Optimal ventricular rate slowing during atrial fibrillation by feedback av nodal-selective vagal stimulation Am J Physiol Heart Circ Physiol, 282(3), p. H1102-10.

26. Tosato, M., K. Yoshida, E. Toft, V. Nekrasas, and J.J. Struijk (2006) Closed-loop control of the heart rate by electrical stimulation of the vagus nerve Med Biol Eng Comput, 44(3), p. 161-9.

27. Elsner, D. and G.A. Riegger (1995) Experimental heart failure produced by rapid ventricular pacing in the dog J Card Fail, 1(3), p. $229-47$.

28. Stambler, B.S., G. Fenelon, R.K. Shepard, H.F. Clemo, and C.M. Guiraudon (2003) Characterization of sustained atrial tachycardia in dogs with rapid ventricular pacing-induced heart failure J Cardiovasc Electrophysiol, 14(5), p. 499-507.

29. Wilson, J.R., P. Douglas, W.F. Hickey, V. Lanoce, N. Ferraro, A. Muhammad, and N. Reichek (1987) Experimental congestive heart 
failure produced by rapid ventricular pacing in the dog: Cardiac effects Circulation, 75(4), p. 857-67.

30. Wallick, D.W., Y. Zhang, T. Tabata, S. Zhuang, K.A. Mowrey, J. Watanabe, N.L. Greenberg, R.A. Grimm, and T.N. Mazgalev (2001) Selective av nodal vagal stimulation improves hemodynamics during acute atrial fibrillation in dogs Am J Physiol Heart Circ Physiol, 281(4), p. H1490-7.

31. Dickerson, L.W., D.J. Rodak, T.J. Fleming, P.J. Gatti, V.J. Massari, J.C. McKenzie, and R.A. Gillis (1998) Parasympathetic neurons in the cranial medial ventricular fat pad on the dog heart selectively decrease ventricular contractility J Auton Nerv Syst, 70(1-2), p. 129-41.

32. Randall, W.C., J.L. Ardell, M.F. O'Toole, and R.D. Wurster (1988) Differential autonomic control of san and avn regions of the canine heart: Structure and function Prog Clin Biol Res, 275, p. 15-31.

33. Yuan, B.X., J.L. Ardell, D.A. Hopkins, and J.A. Armour (1993) Differential cardiac responses induced by nicotine sensitive canine atrial and ventricular neurones Cardiovasc Res, 27(5), p. 760-9.

34. Van Wagoner, D.R. (2011) Chronic vagal nerve stimulation for the treatment of human heart failure: Progress in translating a vision into reality Eur Heart J, 32(7), p.847-55. 


\section{Summary}

This thesis focuses on sensing cardiovascular signals from the vagus nerve and electrically stimulating the vagus nerve for cardiovascular effects. In chapter $\mathbf{2}$, spontaneous vagus nerve activity is analyzed for a cardiac-modulated signal. It is hypothesized that signals from cardiac receptors that fire in phase with the cardiac cycle can be recorded from the whole vagus nerve, using a cuff electrode in the neck region. Digital signal filtering was used to reduce interference from muscular sources and electrical activity of the heart itself. The recorded spontaneous vagus nerve activity was ensemble averaged, i.e. segments of the signal during a cardiac cycle were averaged, to increase signal to noise ratio. A cardiac-modulated vagus nerve activity pattern is indeed found in chapter 2. This pattern may reflect cardiac receptor activity, as it is affected by increased blood pressure and contractility brought about by dobutamine.

Evoked vagus nerve responses were analyzed in anesthetized animals in chapter 3. A component was found that had a long latency, a low stimulation threshold and a high velocity along the nerve. These results led to the hypothesis that the component was an indirect component of a burst of single fibre action potentials, emanating with varying delays from the neural network on the heart. The component was strongly reduced with a tripolar derivation or with the average reference configuration on the centre electrode in a cuff with three rings. In chapter 3 it was proposed that this was due to the second order spatial derivative, which reduces a dispersed component when it creates a close to linear field in the cuff.

In chapter 4 a computer model was used to show that it is possible to record an evoked dispersed component as a result of a burst of single fibre action potentials with varying delays, such as proposed in chapter 3 . This computer model however does not explain why the component was reduced with a second order spatial derivative, since with the model a close to linear field did not result in the cuff from a burst of single fibre action potentials with varying delays.

To further elucidate the origin of the evoked component, two additional experiments were performed in which the recurrent laryngeal nerve was cut. Chapter 5 describes these experiments and their results. The evoked component disappeared after cutting of the recurrent laryngeal nerve. Arguments are presented in chapter 5 for a muscular origin from the larynx or a neuronal origin from the neural network on the heart or the recurrent laryngeal nerve. The physiological origin of the evoked late component remains however as yet unclear. Chapter $\mathbf{6}$ shows that evoked responses may also originate from the brain stem, but that they are difficult to record. 
In chapter 7 the effects of vagus nerve stimulation on the cardiovascular system are described. The results of chapter $\mathbf{8}$ show that selectivity of vagus nerve stimulation for cardiovascular effects can be improved by using a segmented electrode configuration. Finally, chapter 9 shows that the results obtained by vagus nerve sensing and stimulation can be reproduced in awake animals. Challenges when recording from awake pigs are also presented. 


\section{Samenvatting}

Dit proefschrift, getiteld "Meten en stimuleren van de nervus vagus voor kunstmatige controle van het hart", richt zich op het meten van cardiovasculaire signalen van de nervus vagus en het elektrisch stimuleren van de nervus vagus voor cardiovasculaire effecten. In hoofdstuk 2 wordt spontane activiteit in de nervus vagus geanalyseerd voor een cardiaal gemoduleerd signaal. De hypothese wordt gesteld dat signalen van cardiale receptoren, die vuren in fase met de hartcyclus, gemeten kunnen worden aan de nervus vagus met een cuff elektrode in de nek. Digitale filters zijn toegepast op de signalen om de verstoring van spieractiviteit en elektrische activiteit van het hart zelf te verminderen. Het nervus vagus signaal werd opgedeeld in segmenten van een hartcyclus in lengte en daar werd het gemiddelde van bepaald. Hiermee werd de signaal-ruisverhouding verhoogd. Een cardiaal gemoduleerd signaal werd inderdaad gevonden in de spontane nervus vagus activiteit. Dit patroon veranderde bij toegenomen bloeddruk en contractiliteit, veroorzaakt door dobutamine, en is dus mogelijk gerelateerd aan activiteit van cardiovasculaire receptoren

Nervus vagus activiteit, opgewekt door een elektrische puls, is geanalyseerd in geanesthetiseerde dieren in hoofdstuk 3. Een opgewekte component was gevonden met een lange latentietijd, een lage stimulatiedrempel en een hoge snelheid langs de zenuw. Deze resultaten leidden tot de hypothese dat het een indirecte component betrof, ontstaan uit een sommatie van individuele actiepotentialen die met verschillende vertragingen vanaf het neurale netwerk op het hart kwamen. De component werd sterk gereduceerd door een tripolaire afleiding of met de gemiddelde referentie methode op de middelste electrode in een cuff met drie ringen. In hoofdstuk 3 werd gesteld dat dit zou kunnen komen door de tweede orde spatiële afgeleide, die een brede component sterk reduceert als er hierdoor een vrijwel lineair veld ontstaat binnen de cuff.

In hoofdstuk 4 wordt een computermodel gebruikt om aan te tonen dat het mogelijk is om opgewekte activiteit te meten die het resultaat is van een sommatie van individuele actiepotentialen met verschillende vertragingen, zoals was voorgesteld in hoofdstuk 3. Dit computermodel verklaart echter niet waarom de component gereduceerd werd met een tweede orde spatiële afgeleide, omdat er met het model geen lineair veld ontstond binnen de cuff als gevolg van een sommatie van actiepotentialen met verschillende vertragingen.

Om de oorsprong van de component te achterhalen, zijn twee additionele experimenten uitgevoerd waarbij de nervus laryngeus recurrens doorgesneden werd. In hoofdstuk 5 worden deze experimenten en de resultaten beschreven. De eerder beschreven opgewekte late component verdween wanneer de nervus 
laryngeus recurrens doorgesneden werd. In hoofdstuk 5 worden argumenten gegeven voor zowel een musculaire oorsprong van de larynx en een neurale oorsprong van het neurale netwerk op het hart of van de nervus laryngeus recurrens. De fysiologische oorsprong van de opgewekte late component blijft echter onduidelijk. Hoofdstuk 6 toont dat er mogelijk ook opgewekte componenten onstaan vanuit de hersenstam, maar dat deze moeilijk te meten zijn.

In hoofdstuk 7 worden de effecten van nervus vagus stimulatie op het cardiovasculaire systeem beschreven. De resultaten van hoofdstuk 8 laten zien dat selectiviteit van elektrische stimulatie verbeterd kan worden met het gebruik van een gesegmenteerde elektrode. Tot slot laat hoofdstuk 9 zien dan resultaten van geanesthetiseede dieren gereproduceerd kunnen worden in wakkere dieren. De obstakels van metingen in wakkere dieren worden ook in dit hoofdstuk weergegeven. 


\section{Dankwoord}

If you can dream it, you can do it.

Walt Disney

Wat vier jaar geleden begon als een vage toekomstdroom, is werkelijkheid geworden. Het proefschrift is er gekomen. Ik wil iedereen bedanken die me ondersteund heeft in dit bijzondere proces.

Allereerst wil ik mijn promotor en dagelijks begeleider, Peter Veltink, bedanken. Peter, je hebt me ontzettend geholpen in het wetenschappelijk denken en doen. Onze gesprekken gaven me altijd weer nieuwe aanknopingspunten als ik vastliep. Lilian Kornet, mijn assistent-promotor, is een onmisbare steun geweest bij de experimenten en het schrijven van de artikelen. Je had altijd goede tips voor me, waarmee ik in mijn werk kon verbeteren. De bijdrage van Richard Cornelussen was vooral tijdens de experimenten onmisbaar. Richard, je ervaring en enthousiasme hebben de experimenten en de analyse steeds weten uit te breiden en te verbeteren. Rik Buschman ben ik dankbaar voor zijn input op de artikelen en tijdens onze besprekingen. Het CPV team uit Maastricht was ontzettend behulpzaam tijdens de experimenten. Mijn dank gaat in het bijzonder uit naar Joyce Suyk.

Daarnaast wil ik Enrico Marani hartelijk bedanken voor de hulp bij mijn onderzoek. Jouw anatomische kennis en kundige secties brachten de anatomie van de nervus vagus voor onze experimenten in kaart. Zelfs vanuit Frankrijk was je bereid om je hulp te verlenen toen we de nervus laryngeus recurrens probeerden te vinden. Ik wil ook Thiele Kobus bedanken voor het zorgvuldig uitwerken van de anatomische studies tijdens haar Master-opdracht.

Voor de experimenten was ik afhankelijk van heel wat apparatuur. Ed Droog en Marcel Weusthof, bedankt voor het ontwikkelen van de stimulator en de bijbehorende software. De mensen van TMSi wil ik bedanken voor de ondersteuning bij het gebruik van de TMSi versterkers en software, in het bijzonder Jan Peuscher. Het team bij Medtronic dat de elektroden heeft gemaakt zorgde ervoor dat ze ondanks drukke planningen steeds op tijd onze elektroden klaar hadden. In het bijzonder ben ik Paul van Venrooij en Joost Ubachs dankbaar voor hun hulp bij de ontwikkeling van de elektroden. 
Ik heb het erg naar mijn zin gehad bij de groep Biomedische Signalen en Systemen van de Universiteit Twente. Al mijn collega's daar, bedankt voor de gezellige koffiepauzes en lunchmomenten. Bij mijn collega-promovendi kon ik altijd in een feest van herkenning mijn problemen en frustraties kwijt. Het ijs eten bij een promotiedip was voor mij een waardevolle traditie, die hopelijk nog jaren blijft bestaan.

Bij een ernstige promotiedip was een dagje sauna of zilverkleien vaak precies wat ik nodig had. Saskia, Nienke en Heleen, alle leuke uitstapjes die we gedurende deze vier jaar hebben gemaakt vond ik heerlijk en ik hoop dat er nog vele zullen volgen. Saskia, jouw luisterend oor en bemoedigende woorden zijn van grote betekenis voor me geweest. Je staat altijd voor me klaar, wat ik ontzettend waardeer. Nienke, als mede promovenda kon ik altijd bij je terecht voor het uitwisselen van ervaringen en tips. Je hebt me geïnspireerd met je doorzettingsvermogen en passie voor de wetenschap. Heleen, een stukje treinen met jou en ik zag altijd alles weer wat luchtiger in. Bedankt voor al je opbeurende woorden.

In het eerste jaar van mijn promotie heb ik nog met veel plezier geborreld met mijn dispuut Nefertiti. Ook daarna heb ik nog vaak genoten van leuke activiteiten en kerstdiners. Ik wil al mijn lieve dispuutsgenootjes bedanken voor de leuke momenten, die voor de nodige ontspanning en afleiding hebben gezorgd. Sanne, ik heb je steun en onze eet-dates de afgelopen vier jaar bijzonder gewaardeerd. Nicole, met jou kon ik altijd heerlijk discussieren over wetenschappelijke vraagstukken. Bedankt voor je enthousiasme; het werkte vaak aanstekelijk. Anke, je was met je nuchtere instelling en daadkracht een inspiratie voor me.

Ik wil ook mijn familie bedanken voor hun steun en interesse. In het bijzonder mijn ouders, die altijd zoveel vertrouwen in me hebben gehad dat ik nooit lang aan mezelf kon twijfelen, en mijn zus voor haar betrokkenheid.

En lieve Erwin, jij hebt deze vier jaar extra mooi gemaakt. Met jou kon ik alle mooie momenten vieren en de minder leuke snel vergeten. 


\section{Curriculum vitae}

Simone Ordelman was born on February $25^{\text {th }} 1984$ in Nijmegen, the Netherlands. In 2002 she received her VWO diploma from Zwijsen College Veghel. She studied Biomedical Engineering from 2002-2007 at the University of Twente in Enschede, the Netherlands. She received her Bachelor's degree in 2005 after performing her Bachelor assignment at the Roessingh R\&D, which had the topic "Predicting an Epileptic Seizure Based on Heart Rate". In 2006 she performed a traineeship at the Neural Engineering Centre of Case Western Reserve University in Cleveland, Ohio, the United States of America. The topic was "Analyzing EEG data using Self-Organizing Feature Maps". She obtained her Master of Science degree in 2007. From 2007 to 2011 she worked as a PhD student at the department of Biomedical Signals and Systems at the University of Twente, studying the vagus nerve for cardiac signals and effects. In 2011 she became a research scientist at Philips Research. 


\section{List of publications}

\section{Journal articles}

Ordelman, S.C.M.A., Kornet, L., Cornelussen, R., Buschman, H.P.J., and Veltink, P.H. (2010) An indirect component in the evoked compound action potential of the vagal nerve J Neural Eng, vol. 7, pp. 066001

Ordelman, S.C.M.A., Kornet, L., Cornelussen, R., Buschman, H.P.J., and Veltink, P.H. Neural signals modulated with the cardiac cycle can be measured extraneurally from the cervical vagus nerve Submitted

Ordelman, S.C.M.A., Kornet, L., and Veltink, P.H. The average reference method for recording a dispersed evoked signal from peripheral nerves - a modelling study Submitted

Ordelman, S.C.M.A., Kornet, L., Cornelussen, R., Marani, E., and Veltink, P.H Additional experimental data on the origin of the late component in the evoked vagus nerve compound action potential Submitted

Ordelman, S.C.M.A., Kornet, L., Cornelussen, R., Buschman, H.P.J., and Veltink, P.H The cardiac effects of vagus nerve stimulation Submitted

Ordelman, S.C.M.A., Kornet, L., Cornelussen, R., Buschman, H.P.J., and Veltink, P.H Selectivity for specific cardiovascular effects of vagus nerve stimulation with a multi-contact electrode cuff Submitted

\section{Conference proceedings}

Ordelman, S.C.M.A., Kornet, L., Cornelussen, R., Buschman, H.P.J., and Veltink, P.H. (2010) 12-segment cuff is superior to ring cuff for sensing and stimulation of cardiac fibres in the vagal trunk Neuroscience 2010, San Diego

Ordelman, S.C.M.A., Kornet, L., Cornelussen, R., Buschman, H.P.J., and Veltink, P.H. (2010) The evoked compound action potential in the vagal nerve as a potential marker for the cardiovascular system $3^{\text {rd }}$ Dutch Bio-Medical Engineering Conference 2011, Egmond aan Zee

Ordelman, S.C.M.A., Kornet, L., Cornelussen, R., Buschman, H.P.J., and Veltink, P.H. (2009) Average reference recording from the vagal nerve reveals an evoked indirect response In: Proceedings of the 4th International IEEE 
EMBS Conference on Neural Engineering, 29 April - 2 May 2009, Antalya, Turkey. pp. 574-576.

Ordelman, S.C.M.A., Kornet, L., Cornelussen, R., Buschman, H.P.J., and Veltink, P.H. (2009) An evoked indirect response in the cervical vagal nerve In: 4th annual symposium of the IEEE-IMBS Benelux chapter, 9-10 Nov 2009, Enschede, The Netherlands. pp. 28-28.

\section{Book chapter}

Veltink, P.H., Kornet, L., Ordelman, S.C.M.A., Cornelussen, R., Buschman R., Biohybrid Systems for Neurocardiology, in: R. Jung (ed), Biohybrid Systems Nerves, Interfaces and Machines, Wiley-VCH Verlag $\mathrm{GmbH} \& \mathrm{Co}, \mathrm{KGaA}$, 2011, pp. 175-190. 


\section{Stellingen}

Behorende bij het proefschrift van Simone Ordelman, te verdedigen op

27 januari 2012 aan de Universiteit Twente, onder de titel:

\section{"Sensing and stimulation of the vagus nerve for artificial cardiac control"}

1. Gesloten lus controle van het hart is mogelijk door stimulatie van en meten aan de nervus vagus.

2. Spontane neurale activiteit gemeten aan de nervus vagus kan gebruikt worden om het cardiovasculair systeem te monitoren.

3. Selectiviteit van zowel meten als stimuleren van orgaanspecifieke zenuwvezels kan vergroot worden door gebruik te maken van een gesegmenteerde extraneurale cuff elektrode.

4. Selectiviteit voor het meten en stimuleren van cardiale vezels in de nervus vagus is groter in mensen dan in varkens, vanwege de anatomische verschillen.

5. Experimenten waarbij de hypothese niet bevestigd wordt zijn vaak het meest leerzaam.

6. Het is niet eenvoudig wetenschappelijk onderzoek zo te communiceren naar buitenstaanders dat zij niet alleen op de hoogte worden gebracht van de inhoud, maar deze ook werkelijk begrijpen.

7. Zolang in onze maatschappij de verschillen tussen mannen en vrouwen worden benadrukt en gegeneraliseerd, kan men niet verwachten dat mannen en vrouwen gelijk behandeld worden.

8. Mensen die zich verschuilen achter de stelling "Je moet alles een keer geprobeerd hebben", doen dit vaak uit onvermogen of onwil om na te denken over wat hen motiveert.

9. Vele dagelijkse frustraties kunnen worden voorkomen door uit te gaan van goede wil bij de medemens.

10. Als je de tweede keer alles hetzelfde zou doen, heb je niets geleerd. 